\title{
BÄCKLUND TRANSFORMATIONS AND DARBOUX INTEGRABILITY FOR NONLINEAR WAVE EQUATIONS*
}

\author{
JEANNE N. CLELLAND ${ }^{\dagger}$ AND THOMAS A. IVEY ${ }^{\ddagger}$
}

\begin{abstract}
We prove that second-order Monge-Ampère equations for one function of two variables are connected to the wave equation by a Bäcklund transformation if and only if they are integrable by the method of Darboux at second order.
\end{abstract}

Key words. Bäcklund transformations, hyperbolic Monge-Ampère systems, exterior differential systems, Cartan's method of equivalence.

AMS subject classifications. Primary 37K35 58J72; Secondary 35L10, 37K35, 53C10, 58A15

1. Introduction. Roughly speaking, a Bäcklund transformation is a method for generating new solutions for a given partial differential equation by starting with a 'seed' solution to the same (or a different) PDE and solving an auxiliary system of ODEs. Bäcklund's original example was a transformation that produced new pseudospherical surfaces from old, and it is equivalent to the following system:

$$
\begin{aligned}
& v_{x}-u_{x}=\frac{1}{2} \sin ((u+v) / 2), \\
& v_{y}+u_{y}=-\frac{1}{2} \sin ((u-v) / 2) .
\end{aligned}
$$

Given an arbitrary smooth function $u(x, y)$, this overdetermined system for $v$ is inconsistent. However, if $u$ satisfies the sine-Gordon equation $u_{x y}=\sin u$ then the system is consistent, and the function $v(x, y)$, determined up to a constant of integration, will also satisfy the sine-Gordon equation. The transformation works in reverse, too: given a solution $v(x, y)$ for sine-Gordon, the system determines a 1-parameter family of solutions $u(x, y)$ for the same PDE.

It is this type of Bäcklund transformation - connecting solutions of two secondorder Monge-Ampère PDE in the plane, not necessarily the same equation - which is the general subject of this paper. (A second-order Monge-Ampère equation is a PDE where the highest-order derivatives may appear nonlinearly but only in the form of the determinant of the Hessian.) Another important example of this type is the system

$$
\begin{aligned}
& z_{x}-u_{x}=-2 \exp ((u+z) / 2), \\
& z_{y}+u_{y}=\exp ((u-z) / 2)
\end{aligned}
$$

In this example, if $z(x, y)$ satisfies the wave equation (in characteristic coordinates, $z_{x y}=0$ ), then the system determines a 1-parameter family of solutions of Liouville's equation $u_{x y}=e^{u}$, and conversely. Bäcklund transformations of this subtype - where one of the two PDE involved is the standard wave equation - are the specific concern of this paper.

Liouville's equation also has the rare property that it is Darboux-integrable - in other words, it can be solved by the method of Darboux. (This will be defined below.)

\footnotetext{
*Received July 27, 2007; accepted for publication July 14, 2008.

${ }^{\dagger}$ Department of Mathematics, 395 UCB, University of Colorado, Boulder, CO 80309-0395, USA (Jeanne.Clelland@colorado.edu).

${ }^{\ddagger}$ Department of Mathematics, College of Charleston, 66 George St., Charleston, SC 29424-0001, USA (IveyT@cofc.edu).
} 
The main point of this paper is that this is not a coincidence; more precisely, we will prove

TheOREM 1. Let $\left(\mathcal{M}^{5}, \mathcal{I}\right)$ be a hyperbolic Monge-Ampère system. If there is a normal Bäcklund transformation with 1-dimensional fibers linking this system with the wave equation, then the first prolongation of $\mathcal{I}$ is Darboux-integrable. Conversely, if the first prolongation of $\mathcal{I}$ is Darboux-integrable, then near any point $p \in \mathcal{M}$ there is an open set $U \subset \mathcal{M}$ around $p$ such that the restriction of $\mathcal{I}$ to $U$ is linked to the wave equation by a normal Bäcklund transformation.

The technical terms in this theorem must be explained. Any single PDE or system of PDE may be re-cast as an exterior differential system (EDS) or differential ideal (i.e., an ideal, with respect to wedge product, in the ring of differential forms on a manifold, that is also closed under the exterior derivative), in a way that solutions are in one-to-one correspondence with submanifolds to which all the differential forms in the EDS pull back to be zero. (These submanifolds, which must usually also satisfy a nondegeneracy condition, are known as integral manifolds of the EDS.) In particular, a Monge-Ampère equation in the plane can be re-cast as the following type of EDS:

Definition. A Monge-Ampère exterior differential system is a differential ideal $\mathcal{I}$ on a 5 -dimensional manifold $\mathcal{M}$, such that near any point of $\mathcal{M}, \mathcal{I}$ is generated algebraically by one 1 -form $\theta$ and two 2 -forms $\Omega_{1}, \Omega_{2}$. (Hence, $d \theta$ must equal a linear combination of the $\Omega$ 's, plus possibly a wedge product with $\theta$ as factor.) The 1 -form $\theta$ is required to be a contact form, i.e., $\theta \wedge d \theta \wedge d \theta \neq 0$. A Monge-Ampère system is hyperbolic if the $\Omega$ 's may be chosen so that both are decomposable.

For example, for Liouville's equation we may take $\mathcal{M}$ to be $\mathbb{R}^{5}$ with coordinates $x, y, u, p, q$, and let

$$
\theta=d u-p d x-q d y, \quad \Omega_{1}=\left(d p-e^{u} d y\right) \wedge d x, \quad \Omega_{2}=\left(d q-e^{u} d x\right) \wedge d y .
$$

(Note that $d \theta=-\Omega_{1}-\Omega_{2}$.) Given a solution $u=f(x, y)$ of the PDE, we can construct a surface $\Sigma \subset \mathbb{R}^{5}$ such that $i^{*} \theta=0, i^{*} \Omega_{1}=i^{*} \Omega_{2}=0$ (where $i: \Sigma \hookrightarrow \mathbb{R}^{5}$ is the inclusion map) by setting $u=f(x, y), p=f_{x}(x, y)$ and $q=f_{y}(x, y)$. Conversely, any surface $\Sigma$ satisfying $i^{*} \theta=0, i^{*} \Omega_{1}=i^{*} \Omega_{2}=0$ and the nondegeneracy condition $i^{*} d x \wedge d y \neq 0$ is the graph of a solution constructed in this way.

In the body of the paper, we will also use another type of EDS:

Definition. A Pfaffian exterior differential system is a differential ideal $\mathcal{I}$ on an arbitrary manifold $\mathcal{M}$, defined by a vector bundle $I \subset T^{*} \mathcal{M}$, such that a differential form belongs to $\mathcal{I}$ if and only if it is a linear combination of wedge products involving sections of $I$ or their exterior derivatives. (In practice, our Pfaffian systems will be specified by giving a list of 1 -forms that span the fiber of $I$ at each point.) The rank of a Pfaffian system is the rank of the vector bundle.

A Pfaffian system satisfies the Frobenius condition or is said to be integrable if the exterior derivative of any section of $I$ is in the algebraic ideal generated by $I$. Any Frobenius system is locally equivalent to a (possibly underdetermined) system of ordinary differential equations; see Chapter 1 in [8].

Theorem 1 is about relations between exterior differential systems; in particular, we have the following definition from [8]: 
DEFINITION 1.1. A Bäcklund transformation between two exterior differential systems $(\mathcal{M}, \mathcal{I})$ and $(\overline{\mathcal{M}}, \overline{\mathcal{I}})$ is a manifold $\mathcal{B}$ equipped with submersions $\pi: \mathcal{B} \rightarrow \mathcal{M}$ and $\bar{\pi}: \mathcal{B} \rightarrow \overline{\mathcal{M}}$ (see diagram below) and vector bundles $J, \bar{J} \subset T^{*} \mathcal{B}$ such that

(i) the fibers of $\pi$ and $\bar{\pi}$ are transverse in $\mathcal{B}$;

(ii) the rank of $J$ equals the dimension of the fibers of $\pi$, and sections of $J$ pull back to the fibers of $\pi$ to span the cotangent space of each fiber;

(iii) $\bar{J}$ is similarly related to the fibers of $\bar{\pi}$;

(iv) the algebraic ideal $\mathcal{J}$ generated by $\pi^{*} \mathcal{I}$ and sections of $J$ is the same as the algebraic ideal generated by $\bar{\pi}^{*} \overline{\mathcal{I}}$ and sections of $\bar{J}$, and $\mathcal{J}$ is a differential ideal.

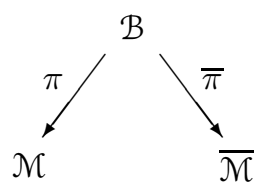

The impact of the last condition is that if $\mathcal{N} \subset \mathcal{M}$ is an integral manifold of $\mathcal{I}$, then sections of $J$ pull back to $\pi^{-1}(\mathcal{N})$ to satisfy the Frobenius condition, so that integral manifolds of $\mathcal{J}$ inside $\pi^{-1}(\mathcal{N})$ may be constructed by solving ODE; moreover, the image under $\bar{\pi}$ of each of these integral manifolds is an integral manifold of $\overline{\mathcal{I}}$. Because the definition is symmetric, this also works in the other direction: given an integral manifold of $\overline{\mathcal{I}}$, we can solve a Frobenius system on the inverse image in $\mathcal{B}$ to obtain a family of integral manifolds of $\mathcal{I}$. For example, given a solution $z(x, y)$ of the wave equation, substitution in (1.2) gives an overdetermined system of ODE for a solution $u(x, y)$ of Liouville's equation, and in this context the Frobenius condition is exactly the compatibility condition for the ODE system.

The condition of normality for Bäcklund transformations, assumed in Theorem 1 , will be explained in $\S 2$.

A hyperbolic Monge-Ampère system is a special case of hyperbolic EDS:

Definition. A hyperbolic EDS of class $k$ is a differential ideal defined on a manifold of dimension $k+4$ that, near any point of the manifold, is generated algebraically by $k 1$-forms and two decomposable 2 -forms.

Associated to a given hyperbolic EDS $\mathcal{I}$ of class $k$ are two characteristic distributions, one corresponding to each decomposable 2-form generator. At each point, the distribution is given by the 2-dimensional subspace of the tangent space annihilated by the $k 1$-forms of the system and the factors of the chosen decomposable 2 -form. (These annihilators form a rank $k+2$ Pfaffian system, known as a characteristic system of $\mathcal{I}$.) A hyperbolic EDS $\mathcal{I}$ is integrable by the method of Darboux, or Darboux-integrable for short, if both characteristic distributions have two independent first integrals, i.e., functions which are constant along all curves tangent to the distribution, and whose differentials are pointwise linearly independent from the 1-forms of $\mathcal{I}$. Such functions are also known as characteristic invariants, since they are constant along integral curves of the distribution.

The Darboux-integrability condition has the virtue that it is easy to check, using only differentiation and linear algebra, by calculating the successive derived systems of each characteristic system. An extensive discussion of hyperbolic EDS and Darbouxintegrability, with worked-out examples, is available in Chapter 6 of [8]. For the purposes of this paper, we will need a few more facts about the method of Darboux: 
- It is known that any Monge-Ampère system which is Darboux-integrable (i.e., has two characteristic invariants for each distribution) is equivalent to the standard wave equation under a contact transformation (see, e.g., Thm. 2.1 in [2]).

- If a hyperbolic Monge-Ampère system $\mathcal{I}$ has a pair of independent first integrals for exactly one of its characteristic distributions, then $\mathcal{I}$ is said to be integrable by the method of Monge or Monge-integrable for short. (The analogous term for hyperbolic EDS of arbitrary class $k$ is Darboux semi-integrable.)

- If a hyperbolic EDS of class $k$ fails to be Darboux-integrable, it is possible that its prolongation, which is a hyperbolic EDS of class $k+2$, is Darbouxintegrable. Thus, a given Monge-Ampère system may lead to a hyperbolic system that is Darboux-integrable only after sufficiently many prolongations. Prolongation of an EDS is essentially the process of adding higher derivatives as new variables and adjoining to the ideal the differential equations satisfied by the higher derivatives. For example, for Liouville's equation we add variables $r$ and $t$ to stand for $u_{x x}$ and $u_{y y}$ respectively, and adjoin the 1 -forms $\theta_{1}=d p-r d x-e^{u} d y, \theta_{2}=d q-$ $e^{u} d x-t d y$. The new ideal is a Pfaffian system on $\mathbb{R}^{7}$ (with coordinates $x, y, u, p, q, r, t$ ) generated by 1 -forms $\theta_{0}, \theta_{1}, \theta_{2}$. (Note that the 2 -forms $\Omega_{1}$ and $\Omega_{2}$ given in (1.3) are now in the ideal generated algebraically by these $\theta_{0}, \theta_{1}, \theta_{2}$.) The set of algebraic generators of the new ideal is completed by computing the exterior derivatives of $\theta_{1}, \theta_{2}$, and these are expressible as linear combinations of the decomposable forms $\Omega_{1}^{\prime}=\left(d r-p e^{u} d y\right) \wedge d x, \Omega_{2}^{\prime}=\left(d t-q e^{u} d x\right) \wedge d y$, modulo multiples of $\theta_{0}, \theta_{1}, \theta_{2}$. (Thus, the new ideal is a hyperbolic EDS of class 3.) Let $\Delta_{1}, \Delta_{2}$ be the corresponding characteristic distributions for the prolongation (i.e., $\Delta_{i}$ is annihilated by $\theta_{0}, \theta_{1}, \theta_{2}$ and the factors of $\Omega_{i}^{\prime}$ ). To see that the system is Darboux-integrable, note that $x, r-\frac{1}{2} p^{2}$ are invariants for $\Delta_{1}$ and $y, t-\frac{1}{2} q^{2}$ are invariants for $\Delta_{2}$.

Remark. Both Darboux-integrability and the transformation (1.2) enable one to express all solutions of Liouville's equation via specifying two arbitrary functions and integrating systems of ODE, and these two solution methods are equivalent, although Darboux's method requires one to solve more ODEs. For, as mentioned above, substituting the wave equation solution $z=f(x)+g(y)$ in (1.2) produces two compatible ODEs for $u(x, y)$. Given an initial value for $u$, these may be integrated simultaneously in the $x$ - and $y$-directions to propagate a solution over an open set in the $x y$-plane. On the other hand, under Darboux's method we obtain ODEs by setting one invariant in each characteristic system to be an arbitrary function of the other, yielding in this case the equations

$$
p_{x}-\frac{1}{2} p^{2}=\phi(x), \quad q_{y}-\frac{1}{2} q^{2}=\psi(y),
$$

which, together with $u_{x}=p$ and $u_{y}=q$, may be integrated to obtain the solution. (In fact, the data for these two methods are related by $\phi=f^{\prime \prime}$ and $\psi=g^{\prime \prime}$, but in other cases we cannot expect the relationship to be this simple.)

Next, we will put Theorem 1 in context with other results both classical and modern. Much of what was known in the 19th century about solving second-order PDE for one function of two variables was summarized in Goursat's treatise [6]. In Volume $2, \S 181$ of that work, we find the following result:

Theorem 2 (Darboux-Goursat). Suppose that a second-order PDE for $z$ as a function of $x, y$ has the property that there exists a Pfaffian system

$$
d F_{i}=\Phi_{i} d \alpha+\Psi_{i} d \beta, \quad 1 \leq i \leq \ell
$$


and formulas

$$
x=V_{1}, \quad y=V_{2}, \quad z=V_{3},
$$

where $\Phi_{i}, \Psi_{i}, V_{1}, V_{2}, V_{3}$ are functions of $F_{1}, \ldots, F_{\ell}, \alpha, \beta, f(\alpha), g(\beta)$ and finitely many of the derivatives of $f$ and $g$, such that (1.4) satisfies the Frobenius condition for arbitrary choices of functions $f$ and $g$, and gives an implicit solution of the PDE for arbitrary choices of initial data for the Frobenius system. Then the PDE is Darbouxintegrable after finitely many prolongations.

The hypotheses of the Darboux-Goursat theorem are fulfilled if the given PDE is linked to the standard wave equation by a Bäcklund transformation. (For, the d'Alembert formula gives solutions of the wave equation $u_{\alpha \beta}=0$ in the form $u=$ $f(\alpha)+g(\beta)$ for arbitrary $f$ and $g$, and the Pfaffian system in the theorem is given by the equations of the Bäcklund transformation.) Compared with the DarbouxGoursat theorem, one direction of our theorem has a stronger hypothesis (essentially, that $\ell=2$ and only first derivatives of $f$ and $g$ are involved) and a stronger conclusion (that at most one prolongation is required to get Darboux-integrability).

In Theorem 6.5.14 in [8] it is shown, by an elementary argument, that Darbouxintegrability of the prolongation implies that there is a Bäcklund transformation between the prolongation (not the original Monge-Ampère system, but one defined by an EDS on a 7-dimensional manifold) and the wave equation (defined by an EDS on a 5-dimensional manifold). However, this asymmetric transformation-relating the 2-jets of solutions of one PDE to the 1-jets of another - is less than satisfying, compared to more symmetrical transformations like (1.2). Our analysis in $\S 4$ shows that it is a much more delicate matter to show that there exists a Bäcklund transformation between two Monge-Ampère systems. We should also note that the argument given in [8] for the other direction (Bäcklund-equivalence to the wave equation implies Darboux-integrability) is unfortunately incorrect, and the proof we give in $\S 3$ of this paper is along completely different lines.

We now briefly outline the rest of the paper. In $\S 2$ we set up the basic machinery required for the first half of the proof, namely, the $G$-structure for Bäcklund transformations originally introduced by the first author in [3]. In $\S 3$ we prove the forward direction in our theorem by following the implications (for the invariants of the $G$ structure) of the existence of a Bäcklund transformation to the wave equation. In $\S 4$ we prove the converse direction by constructing, for any given Darboux-integrable Monge-Ampère equation, an involutive exterior differential system whose solutions are such transformations. In $\S 5$, we discuss our results in the context of earlier classifications of Darboux-integrable equations and of Bäcklund transformations to the wave equation; we also outline an alternate proof technique for the converse direction, which can in some examples be used to establish global existence of the transformation. In $\S 6$ we discuss further steps in our research program.

We are grateful to the referee who read the first version of this paper, and gave us many useful comments and suggestions.

2. $G$-structure for Bäcklund transformations. The material in this section is taken from the first author's paper [3]; additional details may be found there.

Suppose that $(\mathcal{M}, \mathcal{I})$ and $(\overline{\mathcal{N}}, \overline{\mathcal{I}})$ are hyperbolic Monge-Ampère systems, with

$$
\mathcal{I}=\left\{\theta, \Omega_{1}, \Omega_{2}\right\}, \quad \overline{\mathcal{I}}=\left\{\bar{\theta}, \bar{\Omega}_{1}, \bar{\Omega}_{2}\right\} .
$$


As a special case of Definition 1.1, we define a Bäcklund transformation between $(\mathcal{M}, \mathcal{I})$ and $(\overline{\mathcal{M}}, \overline{\mathcal{I}})$ to be a 6 -dimensional submanifold $\mathcal{B} \subset \mathcal{M} \times \overline{\mathcal{M}}$ for which the pullbacks to $\mathcal{B}$ of the forms $\Omega_{1}, \Omega_{2}, \bar{\Omega}_{1}, \bar{\Omega}_{2}$ have the property that

$$
\Omega_{i} \equiv \bar{\Omega}_{i} \quad \bmod \{\theta, \bar{\theta}\}, \quad i=1,2 .
$$

(The vector bundles $J, \bar{J} \subset T^{*} \mathcal{B}$ mentioned in Definition 1.1 are in this case spanned by the pullbacks of $\bar{\theta}$ and $\theta$, respectively.) A Bäcklund transformation is normal in the sense of Theorem 1 if the pullbacks to $\mathcal{B}$ of the 2 -forms $d \theta, d \bar{\theta}$ are linearly independent modulo $\{\theta, \bar{\theta}\}$.

Now let $\mathcal{J}$ be the ideal on $\mathcal{B}$ generated by the pullbacks of $\mathcal{I}$ and $\overline{\mathcal{I}}$; according to the conditions above, $\mathcal{J}$ is generated algebraically by the forms $\{\theta, \bar{\theta}, d \theta, d \bar{\theta}\}$.

Since $\mathcal{I}$ and $\overline{\mathcal{I}}$ are hyperbolic, locally there exist 1-forms $\omega^{1}, \omega^{2}, \omega^{3}, \omega^{4}$ on $\mathcal{B}$ such that $\left\{\theta, \bar{\theta}, \omega^{1}, \omega^{2}, \omega^{3}, \omega^{4}\right\}$ is a coframing of $\mathcal{B}$ (i.e., a set of 1 -forms that restricts, at each point, to be a basis for the cotangent space of $\mathcal{B}$ ) and

$$
\mathcal{J}=\left\{\theta, \bar{\theta}, \omega^{1} \wedge \omega^{2}, \omega^{3} \wedge \omega^{4}\right\} .
$$

(It is important to note that $\theta$ and $\bar{\theta}$ are each separately determined up to a scalar multiple, since each determines the contact structure on a 5-manifold.) Any such coframing has the property that

$$
\begin{array}{ll}
d \theta \equiv A_{1} \omega^{1} \wedge \omega^{2}+A_{2} \omega^{3} \wedge \omega^{4} & \bmod \{\theta, \bar{\theta}\}, \\
d \bar{\theta} \equiv A_{3} \omega^{1} \wedge \omega^{2}+A_{4} \omega^{3} \wedge \omega^{4} & \bmod \{\theta, \bar{\theta}\}
\end{array}
$$

for some nonvanishing functions $A_{1}, A_{2}, A_{3}, A_{4}$. Since $d \theta, d \bar{\theta}$ are required to be linearly independent 2 -forms at each point of $\mathcal{B}$, we must have $A_{1} A_{4}-A_{2} A_{3} \neq 0$.

By rescaling the $\omega^{i}$ and adding multiples of $\theta$ and $\bar{\theta}$ to the $\omega^{i}$ if necessary, we can arrange that

$$
\begin{array}{ll}
d \theta \equiv A_{1} \omega^{1} \wedge \omega^{2}+\omega^{3} \wedge \omega^{4} & \bmod \theta \\
d \bar{\theta} \equiv \omega^{1} \wedge \omega^{2}+A_{2} \omega^{3} \wedge \omega^{4} & \bmod \bar{\theta}
\end{array}
$$

for some nonvanishing functions $A_{1}, A_{2}$ on $\mathcal{B}$ with $A_{1} A_{2} \neq 1$. This coframing is not unique; any other such coframing $\left\{\tilde{\theta}, \tilde{\bar{\theta}}, \tilde{\omega}^{1}, \tilde{\omega}^{2}, \tilde{\omega}^{3}, \tilde{\omega}^{4}\right\}$ has the form

$$
\left[\begin{array}{c}
\tilde{\theta} \\
\tilde{\theta} \\
\tilde{\omega}^{1} \\
\tilde{\omega}^{2} \\
\tilde{\omega}^{3} \\
\tilde{\omega}^{4}
\end{array}\right]=\left[\begin{array}{cccccc}
b_{11} b_{22}-b_{12} b_{21} & 0 & 0 & 0 & 0 & 0 \\
0 & a_{11} a_{22}-a_{12} a_{21} & 0 & 0 & 0 & 0 \\
0 & 0 & a_{11} & a_{12} & 0 & 0 \\
0 & 0 & a_{21} & a_{22} & 0 & 0 \\
0 & 0 & 0 & 0 & b_{11} & b_{12} \\
0 & 0 & 0 & 0 & b_{21} & b_{22}
\end{array}\right]^{-1}\left[\begin{array}{c}
\theta \\
\bar{\theta} \\
\omega^{1} \\
\omega^{2} \\
\omega^{3} \\
\omega^{4}
\end{array}\right]
$$

where $b_{11} b_{22}-b_{12} b_{21} \neq 0, a_{11} a_{22}-a_{12} a_{21} \neq 0$. (The inverse is included for greater ease of computation in carrying out the method of equivalence.) A coframing satisfying (2.1) is called adapted, and the group $G$ of matrices of the above form is called the structure group of the equivalence problem. (In fact, the most general choice of 
structure group would include a discrete component interchanging the distributions $\left\{\omega^{1}, \omega^{2}\right\}$ and $\left\{\omega^{3}, \omega^{4}\right\}$. However, this freedom does not contribute anything crucial to the structure group, and it is easier to work with a connected group.) The associated $G$-structure is the principal $G$-bundle $\mathcal{P} \rightarrow \mathcal{B}$ whose local sections are precisely the adapted coframings over a neighborhood of $\mathcal{B}$.

In [3], it is shown that $\mathcal{P}$ has structure equations

$$
\begin{gathered}
{\left[\begin{array}{c}
d \theta \\
d \bar{\theta} \\
d \omega^{1} \\
d \omega^{2} \\
d \omega^{3} \\
d \omega^{4}
\end{array}\right]=-\left[\begin{array}{cccccc}
\beta_{1}+\beta_{4} & 0 & 0 & 0 & 0 & 0 \\
0 & \alpha_{1}+\alpha_{4} & 0 & 0 & 0 & 0 \\
0 & 0 & \alpha_{1} & \alpha_{2} & 0 & 0 \\
0 & 0 & \alpha_{3} & \alpha_{4} & 0 & 0 \\
0 & 0 & 0 & \beta_{1} & \beta_{2} \\
0 & 0 & 0 & \beta_{3} & \beta_{4}
\end{array}\right] \wedge\left[\begin{array}{c}
\theta \\
\bar{\theta} \\
\omega^{1} \\
\omega^{2} \\
\omega^{3} \\
\omega^{4}
\end{array}\right]} \\
+\left[\begin{array}{ccc}
\theta \wedge\left(A_{1} C_{2} \omega^{1}-A_{1} C_{1} \omega^{2}\right)+A_{1} \omega^{1} \wedge \omega^{2}+\omega^{3} \wedge \omega^{4} \\
\bar{\theta} \wedge\left(A_{2} C_{4} \omega^{3}-A_{2} C_{3} \omega^{4}\right)+\omega^{1} \wedge \omega^{2}+A_{2} \omega^{3} \wedge \omega^{4} \\
B_{1} \theta \wedge \bar{\theta}+C_{1} \omega^{3} \wedge \omega^{4} \\
B_{2} \theta \wedge \bar{\theta}+C_{2} \omega^{3} \wedge \omega^{4} \\
B_{3} \theta \wedge \bar{\theta}+C_{3} \omega^{1} \wedge \omega^{2} \\
B_{4} \theta \wedge \bar{\theta}+C_{4} \omega^{1} \wedge \omega^{2}
\end{array}\right]
\end{gathered}
$$

for some functions $A_{i}, B_{i}, C_{i}$ and 1 -forms $\alpha_{i}, \beta_{i}$ on $\mathcal{P}$. These equations are chosen so that the matrix in (2.3) takes values in the Lie algebra $\mathfrak{g}$ of $G$; this is a standard step in the method of equivalence. (See [5] for details.)

The 1 -forms $\alpha_{i}, \beta_{i}$ are linearly independent from each other and from $\theta, \bar{\theta}, \omega^{i}$; they are called pseudoconnection forms, or more concisely (but imprecisely) connection forms on $\mathcal{P}$. They are well-defined only up to transformations of the form

$$
\begin{array}{rlrl}
\alpha_{1} & \mapsto \alpha_{1}+r_{1} \omega^{1}+r_{2} \omega^{2}, & & \beta_{1} \mapsto \beta_{1}+s_{1} \omega^{3}+s_{2} \omega^{4}, \\
\alpha_{2} \mapsto \alpha_{2}+r_{2} \omega^{1}+r_{3} \omega^{2}, & \beta_{2} \mapsto \beta_{2}+s_{2} \omega^{3}+s_{3} \omega^{4}, \\
\alpha_{3} \mapsto \alpha_{3}+r_{4} \omega^{1}-r_{1} \omega^{2}, & \beta_{3} \mapsto \beta_{3}+s_{4} \omega^{3}-s_{1} \omega^{4}, \\
\alpha_{4} \mapsto \alpha_{4}-r_{1} \omega^{1}-r_{2} \omega^{2}, & \beta_{4} \mapsto \beta_{4}-s_{1} \omega^{3}-s_{2} \omega^{4} .
\end{array}
$$

Remark. The coefficients $A_{i}, B_{i}, C_{i}$ are called torsion functions. They may be interpreted as the components of well-defined tensors associated to the Bäcklund transformation, as follows.

A hyperbolic Monge-Ampère system naturally equips the underlying manifold $\mathcal{M}^{5}$ with a line bundle $L \subset T^{*} \mathcal{M}$ and two rank 3 characteristic bundles $K_{1}, K_{2} \subset T^{*} \mathcal{M}$ whose intersection is $L$. (The generator 1 -form $\theta$ is a section of $L$, and the factors of the decomposable generator 2 -forms $\Omega_{1}$ and $\Omega_{2}$ span a complement of $L$ within $K_{1}$ and $K_{2}$, respectively.) The $G$-structure for the Bäcklund transformation shows that $\mathcal{B}^{6}$ is equipped with a well-defined splitting of its cotangent bundle:

$$
T^{*} \mathcal{B}=L \oplus \bar{L} \oplus W_{1} \oplus W_{2},
$$


where $L$ and $\bar{L}$ are the pullbacks of the Monge-Ampère line bundles from $\mathcal{M}$ and $\overline{\mathcal{M}}$ respectively, and $W_{1}, W_{2}$ are spanned by $\omega^{1}, \omega^{2}$ and $\omega^{3}, \omega^{4}$ respectively. The normal Bäcklund condition implies that

$$
\pi^{*} K_{i}=L \oplus W_{i}, \quad \bar{\pi}^{*} \bar{K}_{i}=\bar{L} \oplus W_{i}, \quad i=1,2 .
$$

The splitting (2.5) induces a corresponding splitting of $\Lambda^{2} T^{*} \mathcal{B}$, whose summands include the line bundles $L \otimes \bar{L}, \Lambda^{2} W_{1}$ and $\Lambda^{2} W_{2}$. We may then define a natural map from sections of $L$ to sections of $\Lambda^{2} W_{1}$, given by

$$
\delta_{0}: \theta \mapsto \text { projection of } d \theta \text { into } \Lambda^{2} W_{1} .
$$

But this map is linear under multiplication by functions, and so gives a well-defined map between the corresponding vector bundles. The structure equations (2.3) show that $A_{1} \omega^{1} \wedge \omega^{2}$ is the value of $\delta_{0}$ applied to the first member $\theta$ of the coframe. Hence, $A_{1}$ is the component, with respect to the given coframe, of a well-defined tensor in $L^{*} \otimes \Lambda^{2} W_{1}$. Similarly, $A_{2}$ is the component of a well-defined tensor in $\bar{L}^{*} \otimes \Lambda^{2} W_{2}$.

We may similarly define a map on sections of $W_{1}$ by

$$
\delta_{1}: \omega \mapsto \text { projection of } d \omega \text { into } L_{1} \otimes L_{2},
$$

which again is linear under multiplication by functions. The structure equations show that $B_{1} \theta \wedge \bar{\theta}$ and $B_{2} \theta \wedge \bar{\theta}$ give the value of $\delta_{1}$ on the basis sections $\omega^{1}, \omega^{2}$ of $W_{1}$, respectively. Thus, the vector $\left[\begin{array}{ll}B_{1} & B_{2}\end{array}\right]$ gives the components, with respect to the coframe, of a well-defined tensor in $W_{1}^{*} \otimes L_{1} \otimes L_{2}$. In a similar way, we see that $\left[\begin{array}{ll}B_{3} & B_{4}\end{array}\right]$ are the components of a tensor in $W_{2}^{*} \otimes L_{1} \otimes L_{2},\left[\begin{array}{ll}C_{1} & C_{2}\end{array}\right]$ are the components of a tensor in $W_{1}^{*} \otimes \Lambda^{2} W_{2}$, and $\left[\begin{array}{ll}C_{3} & C_{4}\end{array}\right]$ are the components of a tensor in $W_{2}^{*} \otimes \Lambda^{2} W_{1}$, all defined by taking the exterior derivative of a section and projecting into the appropriate summand of $\Lambda^{2} T^{*} \mathcal{B}$.

The following results are proved in [3]:

Proposition 2.1. The vectors $\left[\begin{array}{ll}B_{1} & B_{2}\end{array}\right],\left[\begin{array}{ll}B_{3} & B_{4}\end{array}\right],\left[\begin{array}{ll}C_{1} & C_{2}\end{array}\right],\left[\begin{array}{ll}C_{3} & C_{4}\end{array}\right]$ are relative invariants: given any point $m \in \mathcal{B}$, they are each either zero for every adapted coframing at $m$, or nonzero for every adapted coframing at $m$.

Proposition 2.2. If $\left[\begin{array}{ll}C_{1} & C_{2}\end{array}\right]=\left[\begin{array}{ll}C_{3} & C_{4}\end{array}\right]=\left[\begin{array}{ll}0 & 0\end{array}\right]$, then $\left[\begin{array}{ll}B_{1} & B_{2}\end{array}\right]=\left[\begin{array}{ll}B_{3} & B_{4}\end{array}\right]=$ $\left[\begin{array}{ll}0 & 0\end{array}\right]$ as well, and $(\mathcal{M}, \mathcal{I})$ and $(\overline{\mathcal{M}}, \overline{\mathcal{I}})$ are each contact equivalent to the Monge-Ampère system representing the standard wave equation.

Proposition 2.3. If $\left[\begin{array}{ll}C_{1} & C_{2}\end{array}\right]=\left[\begin{array}{ll}0 & 0\end{array}\right]$ (resp., $\left[\begin{array}{ll}C_{3} & C_{4}\end{array}\right]=\left[\begin{array}{ll}0 & 0\end{array}\right]$ ), then $\left[\begin{array}{ll}B_{1} & B_{2}\end{array}\right]=$ $\left[\begin{array}{ll}0 & 0\end{array}\right]$ (resp., $\left.\left[\begin{array}{ll}B_{3} & B_{4}\end{array}\right]=\left[\begin{array}{ll}0 & 0\end{array}\right]\right)$ as well, and $(\mathcal{M}, \mathcal{I})$ and $(\overline{\mathcal{M}}, \overline{\mathcal{I}})$ are each Mongeintegrable.

Proposition 2.4. Suppose that the vectors $\left[\begin{array}{ll}C_{1} & C_{2}\end{array}\right]$ and $\left[\begin{array}{ll}C_{3} & C_{4}\end{array}\right]$ are both nonzero. Then the vectors $\left[\begin{array}{ll}B_{1} & B_{2}\end{array}\right]$ and $\left[\begin{array}{ll}B_{3} & B_{4}\end{array}\right]$ are either both zero or both nonzero.

If $\left[\begin{array}{ll}B_{1} & B_{2}\end{array}\right]=\left[\begin{array}{ll}B_{3} & B_{4}\end{array}\right]=\left[\begin{array}{ll}0 & 0\end{array}\right]$, then the differential ideal generated by $\left\{\omega^{1}, \omega^{2}, \omega^{3}, \omega^{4}\right\}$ is a Frobenius system. (The converse is true as well.) It follows that locally, there exists a 4 -manifold $\mathcal{V}$ which is a quotient of $\mathcal{B}$ and for which the 1 forms $\omega^{1}, \omega^{2}, \omega^{3}, \omega^{4}$ are semi-basic for the projection $\rho: \mathcal{B} \rightarrow \mathcal{V}$. (Here "locally" refers to the fact that any point in $\mathcal{B}$ has a neighborhood which possesses such a quotient, 
and "semi-basic" means that the restrictions of the $\omega^{i}$ to the fibers of the projection vanish identically. See [8] for details.) In fact, this quotient factors through each of the quotients $\pi: \mathcal{B} \rightarrow \mathcal{M}$ and $\bar{\pi}: \mathcal{B} \rightarrow \overline{\mathcal{M}}$, as shown by the following commutative diagram.

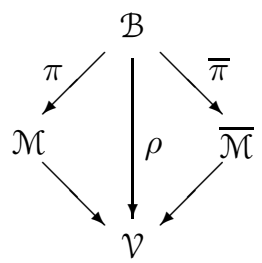

The vanishing of the vector $\left[\begin{array}{ll}B_{1} & B_{2}\end{array}\right]$ implies that the span of $\left\{\omega^{1}, \omega^{2}\right\}$ is unchanged along the fibers of $\rho$, and is thus the pullback via $\rho$ of a well-defined rank 2 sub-bundle of $T^{*} \mathcal{V}$. When the vector $\left[B_{3} \quad B_{4}\right]$ also vanishes, the ideal $\left\{\omega^{1} \wedge \omega^{2}, \omega^{3} \wedge \omega^{4}\right\}$ is the pullback via $\rho$ of a well-defined hyperbolic system $\mathcal{H}$ of class 0 on $\mathcal{V}$, and $\mathcal{I}, \overline{\mathcal{I}}$ are both integrable extensions of $\mathcal{H}$.

Bäcklund transformations of this type are called holonomic. One can test whether a Bäcklund transformation is holonomic by checking whether the Pfaffian system on $\mathcal{B}$ spanned by the intersection of the basic forms for $\pi$ with the basic forms for $\bar{\pi}$ is Frobenius. Note that the basic forms for $\pi$ are spanned by the Cartan system ${ }^{1}$ of $\mathcal{I}$, and the basic forms for $\bar{\pi}$ are spanned by the Cartan system of $\overline{\mathcal{I}}$.

Holonomic Bäcklund transformations are generally considered less interesting than non-holonomic Bäcklund transformations because of their limited capacity to generate new solutions, which we now explain. Given an integral surface $\mathcal{N}$ of $(\mathcal{M}, \mathcal{I})$, solving the Frobenius system $\mathcal{J}$ on $\pi^{-1}(\mathcal{N})$ produces a 1-parameter family of integral surfaces $\overline{\mathcal{N}}_{\lambda}$ of $(\overline{\mathcal{M}}, \overline{\mathcal{I}})$. Reversing the process, beginning with any one of the integral manifolds $\overline{\mathcal{N}}_{\lambda}$, in turn produces a 1-parameter family of integral surfaces $\mathcal{N}_{\lambda, \mu}$ of $(\mathcal{M}, \mathcal{I})$. In general, this results in a 2-parameter family of integral surfaces of $(\mathcal{M}, \mathcal{I})$, and iterating the process produces an ever-increasing family of new integral surfaces for both Monge-Ampère systems.

For example, consider the system (1.2). If we substitute the trivial solution $z(x, y)=0$ of the wave equation into (1.2), the resulting overdetermined PDE system for $u$ yields the 1-parameter family of solutions

$$
u(x, y)=-2 \ln \left(-x-\frac{1}{2} y-c_{1}\right)
$$

to Liouville's equation. Reversing the process, substituting (2.6) into (1.2) produces a PDE system for $z$ which has a 2-parameter family of solutions:

$$
z(x, y)=2 \ln \left(-y-c_{2}\right)-2 \ln \left(2 x+2 c_{1}-c_{2}\right) .
$$

Finally, substituting (2.7) into (1.2) produces a PDE system for $u$ which has a 3parameter family of solutions:

$$
u(x, y)=-2 \ln \left(c_{3} x y+\left(c_{2} c_{3}-1\right) x+\left(c_{1} c_{1}-\frac{1}{2} c_{2} c_{3}-\frac{1}{2}\right) y+\left(c_{1} c_{2} c_{3}-\frac{1}{2} c_{2}^{2} c_{3}-c_{1}\right)\right) .
$$

It is clear that the solutions (2.6) form a proper subset of the solutions (2.8), since the argument of the latter contains an $x y$ term.

For the system (1.2), and for non-holonomic Bäcklund transformations in general, successive iterations of this process continue to produce new solutions. However, if the

\footnotetext{
${ }^{1}$ The Cartan system for a given $\operatorname{EDS} \mathcal{I}$ is the smallest Frobenius system that contains $\mathcal{I}$.
} 
Bäcklund transformation is holonomic, then all integral surfaces of $(\mathcal{N}, \mathcal{I})$ and $(\overline{\mathcal{M}}, \overline{\mathcal{I}})$ produced by this process must lie in the inverse image of a single integral surface of $(\mathcal{V}, \mathcal{H})$. It follows that successive iterations can produce no more than a 1-parameter family of integral surfaces for each Monge-Ampère system.

3. Proof that Bäcklund implies Darboux. Now suppose that we have a Bäcklund transformation as in $\S 2$, and that the Monge-Ampère system $\left(\mathcal{M}^{5}, \mathcal{I}\right)$ is contact equivalent to the standard wave equation $Z_{X Y}=0$. We can choose local coordinates $(X, Y, Z, P, Q)$ on $\mathcal{M}$ such that $\mathcal{I}$ is generated by the forms

$$
\theta=d Z-P d X-Q d Y, \quad \Omega_{1}=d X \wedge d P, \quad \Omega_{2}=d Y \wedge d Q .
$$

There is a unique local section $\sigma=\left(\theta, \bar{\theta}, \omega^{1}, \omega^{2}, \omega^{3}, \omega^{4}\right): \mathcal{B} \rightarrow \mathcal{P}$ satisfying

$$
\begin{aligned}
\theta & =d Z-P d X-Q d Y, \\
\omega^{1} & =d X+C_{1} \theta, \\
\omega^{2} & =d P+C_{2} \theta, \\
\omega^{3} & =d Y+C_{3} \theta, \\
\omega^{4} & =d Q+C_{4} \theta
\end{aligned}
$$

for some functions $C_{i}$ on $\mathcal{B}$. (Note that, because specifying this portion of the coframing determines a unique local section of $\mathcal{P}$, the 1 -form $\bar{\theta}$ is also uniquely determined.) The functions $C_{i}$ are the pullbacks under $\sigma$ of the corresponding torsion functions, and this coframing has $A_{1} \equiv 1$. When the structure equations (2.3) are pulled back to $\mathcal{B}$ via $\sigma$, the 1 -forms $\alpha_{i}, \beta_{i}$-which were linearly independent from the 1 -forms $\theta, \bar{\theta}, \omega^{i}$ on $\mathcal{P}$-must pull back to some linear combinations of these 1 -forms.

Now we embark on the process of comparing the structure equations (2.3) to those for the explicit coframing above. First, note that

$$
0=d(d X)=d\left(\omega^{1}-C_{1} \theta\right) \equiv-\left(\alpha_{1} \wedge \omega^{1}+\alpha_{2} \wedge \omega^{2}+C_{1} \omega^{1} \wedge \omega^{2}\right) \bmod \theta .
$$

Therefore, by choosing $r_{2}, r_{3}$ appropriately in (2.4), we may assume that

$$
\alpha_{1}=a_{10} \theta+a_{11} \omega^{1}+\frac{1}{2} C_{1} \omega^{2}, \quad \alpha_{2}=a_{20} \theta-\frac{1}{2} C_{1} \omega^{1}
$$

for some functions $a_{10}, a_{11}, a_{20}$ on $\mathcal{B}$. Similar considerations of $d(d P), d(d Y), d(d Q)$ modulo $\theta$ yield similar expressions for the remaining connection forms:

$$
\begin{array}{lll}
\alpha_{1}=a_{10} \theta+a_{11} \omega^{1}+\frac{1}{2} C_{1} \omega^{2}, & \beta_{1}=b_{10} \theta+b_{13} \omega^{3}+\frac{1}{2} C_{3} \omega^{4}, \\
\alpha_{2}=a_{20} \theta-\frac{1}{2} C_{1} \omega^{1}, & \beta_{2}=b_{20} \theta-\frac{1}{2} C_{3} \omega^{3}, \\
\alpha_{3}=a_{30} \theta+\frac{1}{2} C_{2} \omega^{2}, & \beta_{3}=b_{30} \theta+\frac{1}{2} C_{4} \omega^{4}, \\
\alpha_{4}=a_{40} \theta-\frac{1}{2} C_{2} \omega^{1}+a_{42} \omega^{2}, & \beta_{4}=b_{40} \theta-\frac{1}{2} C_{4} \omega^{3}+b_{44} \omega^{4} .
\end{array}
$$

Next, a straightforward computation shows that for the coframing (3.1),

$$
d \theta=\theta \wedge\left(C_{2} \omega^{1}-C_{1} \omega^{2}+C_{4} \omega^{3}-C_{3} \omega^{4}\right)+\omega^{1} \wedge \omega^{2}+\omega^{3} \wedge \omega^{4} .
$$

Comparing this with the first structure equation in (2.3) yields

$$
b_{13}=\frac{3}{2} C_{4}, \quad b_{44}=-\frac{3}{2} C_{3} .
$$


In order to continue this comparison, we need to introduce derivatives of the functions $A_{2}, C_{i}$. So, set

$$
\begin{aligned}
& d A_{2}=A_{2,0} \theta+A_{2, \overline{0}} \bar{\theta}+A_{2,1} \omega^{1}+A_{2,2} \omega^{2}+A_{2,3} \omega^{3}+A_{2,4} \omega^{4}, \\
& d C_{1}=C_{1,0} \theta+C_{1, \overline{0}} \bar{\theta}+C_{1,1} \omega^{1}+C_{1,2} \omega^{2}+C_{1,3} \omega^{3}+C_{1,4} \omega^{4} \\
& d C_{2}=C_{2,0} \theta+C_{2, \overline{0}} \bar{\theta}+C_{2,1} \omega^{1}+C_{2,2} \omega^{2}+C_{2,3} \omega^{3}+C_{2,4} \omega^{4} \\
& d C_{3}=C_{3,0} \theta+C_{3, \overline{0}} \bar{\theta}+C_{3,1} \omega^{1}+C_{3,2} \omega^{2}+C_{3,3} \omega^{3}+C_{3,4} \omega^{4} \\
& d C_{4}=C_{4,0} \theta+C_{4, \overline{0}} \bar{\theta}+C_{4,1} \omega^{1}+C_{4,2} \omega^{2}+C_{4,3} \omega^{3}+C_{4,4} \omega^{4}
\end{aligned}
$$

Comparing the structure equations (2.3) for $d \omega^{i}$ with the derivatives of the explicit forms $\omega^{i}$ in (3.1) yields

$$
\begin{array}{ll}
a_{10}=C_{1,1}-C_{1} C_{2}, & b_{10}=C_{3,3}-C_{3} C_{4} \\
a_{20}=C_{1,2}+C_{1}^{2}, & b_{20}=C_{3,4}+C_{3}^{2}, \\
a_{30}=C_{2,1}-C_{2}^{2}, & b_{30}=C_{4,3}-C_{4}^{2}, \\
a_{40}=C_{2,2}+C_{1} C_{2}, & b_{40}=C_{4,4}+C_{3} C_{4},
\end{array}
$$

in addition to the following relations among the torsion and its derivatives:

$$
\begin{array}{lll}
B_{1}=-C_{1, \overline{0}}, & C_{1,3}=C_{1} C_{4}, & C_{3,1}=C_{2} C_{3}, \\
B_{2}=-C_{2, \overline{0}}, & C_{1,4}=-C_{1} C_{3}, & C_{3,2}=-C_{1} C_{3}, \\
B_{3}=-C_{3, \overline{0}}, & C_{2,3}=C_{2} C_{4}, & C_{4,1}=C_{2} C_{4}, \\
B_{4}=-C_{4, \overline{0}}, & C_{2,4}=-C_{2} C_{3}, & C_{4,2}=-C_{1} C_{4} .
\end{array}
$$

While we don't have an explicit coordinate representation for $\bar{\theta}$, we can still explore the consequences of $d(d \bar{\theta})=0$. Computing $d(d \bar{\theta}) \equiv 0$ modulo $\bar{\theta}$ yields

$$
\begin{gathered}
a_{11}=\frac{-2 A_{2,1}+C_{2}\left(A_{2}+2\right)}{2 A_{2}}, \quad a_{42}=\frac{-2 A_{2,2}-C_{1}\left(A_{2}+2\right)}{2 A_{2}}, \\
A_{2,0}=A_{2}\left(C_{3,3}+C_{4,4}-C_{1,1}-C_{2,2}\right) .
\end{gathered}
$$

Then computing $d(d \bar{\theta}) \equiv 0$ modulo $\theta, \omega^{1}, \omega^{2}$ yields

$$
A_{2, \overline{0}}=C_{1,2}+C_{2,2}-A_{2}\left(C_{3,3}+C_{4,4}\right)-\frac{C_{1}}{A_{2}} A_{2,1}-\frac{C_{2}}{A_{2}} A_{2,2}-C_{3} A_{2,3}-C_{4} A_{2,4} .
$$

(Note that the fact that $\bar{\theta}$ is a contact form implies that $A_{2}$ cannot be zero.)

At this point, all coefficients in the structure equations (2.3) have been expressed in terms of the functions $A_{2}, C_{1}, C_{2}, C_{3}, C_{4}$ and their first derivatives. In addition, we have relations among the derivatives that amount to an overdetermined PDE system for these five functions on $\mathcal{B}$. Necessary compatibility conditions for this system may be found by computing $d\left(d A_{2}\right)=d\left(d C_{i}\right)=0$. In particular, computing

$$
\begin{aligned}
& d\left(d C_{1}\right) \equiv d\left(d C_{2}\right) \equiv 0 \quad \bmod \theta, \bar{\theta}, \omega^{1}, \omega^{2}, \\
& d\left(d C_{3}\right) \equiv d\left(d C_{4}\right) \equiv 0 \quad \bmod \theta, \bar{\theta}, \omega^{3}, \omega^{4}
\end{aligned}
$$


yields

$$
\begin{aligned}
& C_{1,0}=-A_{2} C_{1, \overline{0}}-C_{2} C_{1,2}+C_{1}\left(-C_{1,1}+C_{3,3}+C_{4,4}\right), \\
& C_{2,0}=-A_{2} C_{2, \overline{0}}-C_{1} C_{2,1}+C_{2}\left(-C_{2,2}+C_{3,3}+C_{4,4}\right), \\
& C_{3,0}=-C_{3, \overline{0}}-C_{4} C_{3,4}+C_{3}\left(C_{1,1}+C_{2,2}-C_{3,3}\right), \\
& C_{4,0}=-C_{4, \overline{0}}-C_{3} C_{4,3}+C_{4}\left(C_{1,1}+C_{2,2}-C_{4,4}\right) .
\end{aligned}
$$

At this point, we have derived all the relations among the torsion functions on $\mathcal{B}$ and their derivatives that will be needed in order to prove that $(\overline{\mathcal{M}}, \overline{\mathcal{I}})$ is Darbouxintegrable after at most one prolongation. The proof of Darboux-integrability is divided into two main cases. In $\S 3.1$, we prove Darboux-integrability under the assumption that the vectors $\left[\begin{array}{ll}C_{1} & C_{2}\end{array}\right],\left[\begin{array}{ll}C_{3} & C_{4}\end{array}\right]$ are both nonzero. This case is further divided into three subcases, depending on the ranks of certain Frobenius systems that arise during the proof. Precise statements of the results are contained in Propositions $3.1,3.2$, and 3.3. In $\S 3.2$, we prove Darboux-integrability under the assumption that exactly one of the vectors $\left[\begin{array}{ll}C_{1} & C_{2}\end{array}\right],\left[\begin{array}{ll}C_{3} & C_{4}\end{array}\right]$ vanishes; the precise result is contained in Proposition 3.4. As noted in $\S 2$, it is not necessary to consider the case where $\left[\begin{array}{ll}C_{1} & C_{2}\end{array}\right],\left[\begin{array}{ll}C_{3} & C_{4}\end{array}\right]$ both vanish, since in that case both Monge-Ampère systems are contact equivalent to the standard wave equation.

3.1. Case 1: $\left[\begin{array}{ll}C_{1} & C_{2}\end{array}\right],\left[\begin{array}{ll}C_{3} & C_{4}\end{array}\right]$ are both nonzero. Without loss of generality, we may assume that $C_{2}$ and $C_{4}$ are nonzero. Consider the exterior derivatives of the ratios $\frac{C_{1}}{C_{2}}$ and $\frac{C_{3}}{C_{4}}$. A straightforward computation shows that

$$
d\left(\frac{C_{1}}{C_{2}}\right) \equiv 0 \quad \bmod \theta, \bar{\theta}, \omega^{1}, \omega^{2}
$$

therefore, $d\left(\frac{C_{1}}{C_{2}}\right)$ must lie in the last derived system of $\mathcal{K}_{1}=\left\{\theta, \bar{\theta}, \omega^{1}, \omega^{2}\right\}$-i.e., the largest integrable subsystem of $\mathcal{K}_{1}$, denoted by $\mathcal{K}_{1}^{(\infty)}$. Similarly,

$$
d\left(\frac{C_{3}}{C_{4}}\right) \equiv 0 \quad \bmod \theta, \bar{\theta}, \omega^{3}, \omega^{4},
$$

so $d\left(\frac{C_{3}}{C_{4}}\right)$ must lie in the last derived system of $\mathcal{K}_{2}=\left\{\theta, \bar{\theta}, \omega^{3}, \omega^{4}\right\}$.

First, consider the system $\mathcal{K}_{1}$. In order to compute its first derived system $\mathcal{K}_{1}^{(1)}$, we must find those 1 -forms in $\mathcal{K}_{1}$ whose exterior derivatives are zero modulo the linear span of the 1-forms in $\mathcal{K}_{1}$. To this end, we compute:

$$
\left.\begin{array}{rl}
d \theta & \equiv \omega^{3} \wedge \omega^{4} \\
d \bar{\theta} & \equiv A_{2} \omega^{3} \wedge \omega^{4} \\
d \omega^{1} & \equiv C_{1} \omega^{3} \wedge \omega^{4} \\
d \omega^{2} & \equiv C_{2} \omega^{3} \wedge \omega^{4}
\end{array}\right\} \quad \bmod \mathcal{K}_{1}
$$

Therefore, $\mathcal{K}_{1}^{(1)}=\left\{\bar{\theta}-A_{2} \theta, \omega^{1}-C_{1} \theta, \omega^{2}-C_{2} \theta\right\}$. Observe that

$$
\omega^{1}-C_{1} \theta=d X, \quad \omega^{2}-C_{2} \theta=d P .
$$


Therefore, the rank 2 subsystem $\left\{\omega^{1}-C_{1} \theta, \omega^{2}-C_{2} \theta\right\}=\{d X, d P\}$ of $\mathcal{K}_{1}^{(1)}$ is integrable, and the next derived system $\mathcal{K}_{1}^{(2)}$ (i.e., the first derived system of $\mathcal{K}_{1}^{(1)}$ ) contains this rank 2 system. The only question is whether, in fact, $\mathcal{K}_{1}^{(2)}=\mathcal{K}_{1}^{(1)}$-i.e., whether $\mathcal{K}_{1}^{(1)}$ itself is integrable. In either case, we will have $\mathcal{K}_{1}^{(2)}=\mathcal{K}_{1}^{(\infty)}$. A computation shows that

$d\left(\bar{\theta}-A_{2} \theta\right) \equiv \theta \wedge\left[\left(A_{2,3}+A_{2} C_{4}\left(A_{2}-1\right)\right) \omega^{3}+\left(A_{2,4}-A_{2} C_{3}\left(A_{2}-1\right)\right) \omega^{4}\right] \quad \bmod \mathcal{K}_{1}^{(1)}$,

so the rank of $\mathcal{K}_{1}^{(\infty)}$ is either 3 or 2 , depending on whether or not the 1 -form in brackets vanishes.

Similarly, we can compute that

$$
\mathcal{K}_{2}^{(1)}=\left\{\bar{\theta}-\theta, \omega^{3}-C_{3} \theta, \omega^{4}-C_{4} \theta\right\}=\{\bar{\theta}-\theta, d Y, d Q\} .
$$

So $\mathcal{K}_{2}^{(\infty)}$ contains the rank 2 subsystem $\left\{\omega^{3}-C_{3} \theta, \omega^{4}-C_{4} \theta\right\}=\{d Y, d Q\}$, and a computation shows that

$$
d(\bar{\theta}-\theta) \equiv-\theta \wedge\left[\frac{\left(A_{2,1}+C_{2}\left(A_{2}-1\right)\right)}{A_{2}} \omega^{1}+\frac{\left(A_{2,2}-C_{1}\left(A_{2}-1\right)\right)}{A_{2}} \omega^{2}\right] \quad \bmod \mathcal{K}_{2}^{(1)}
$$

So the rank of $\mathcal{K}_{2}^{(\infty)}$ is either 3 or 2 , depending on whether or not the 1 -form in brackets vanishes.

Now we must divide into cases depending on the ranks of these derived systems.

3.1.1. Case 1.1: $\mathcal{K}_{1}^{(\infty)}$ and $\mathcal{K}_{2}^{(\infty)}$ both have rank 3. In this case, we have the following result:

Proposition 3.1. If $\left[\begin{array}{ll}C_{1} & C_{2}\end{array}\right],\left[\begin{array}{ll}C_{3} & C_{4}\end{array}\right]$ are both nonzero and $\mathcal{K}_{1}^{(\infty)}$ and $\mathcal{K}_{2}^{(\infty)}$ both have rank 3 , then the system $(\overline{\mathcal{M}}, \overline{\mathcal{I}})$ is contact equivalent to the standard wave equation.

Proof. By Theorem 2.1 of [2], it suffices to show that $(\overline{\mathcal{M}}, \overline{\mathcal{I}})$ is Darboux-integrable, i.e., that each of the characteristic systems $\left\{\bar{\theta}, \omega^{1}, \omega^{2}\right\}$ and $\left\{\bar{\theta}, \omega^{3}, \omega^{4}\right\}$-which are well-defined on $\overline{\mathcal{M}}$ even though the 1 -forms $\omega^{i}$ are not - contains a rank 2 integrable subsystem.

The hypothesis that $\mathcal{K}_{1}^{(\infty)}$ and $\mathcal{K}_{2}^{(\infty)}$ both have rank 3 implies that the expressions (3.5) and (3.6) must both vanish identically; therefore,

$$
\begin{array}{ll}
A_{2,1}=-C_{2}\left(A_{2}-1\right), & A_{2,2}=C_{1}\left(A_{2}-1\right), \\
A_{2,3}=-A_{2} C_{4}\left(A_{2}-1\right), & A_{2,4}=A_{2} C_{3}\left(A_{2}-1\right) .
\end{array}
$$

Using these conditions, a straightforward computation shows that

$$
\begin{aligned}
& \left\{\bar{\theta}, \omega^{1}, \omega^{2}\right\}^{(1)}=\left\{A_{2} \omega^{1}-C_{1} \bar{\theta}, A_{2} \omega^{2}-C_{2} \bar{\theta}\right\}, \\
& \left\{\bar{\theta}, \omega^{3}, \omega^{4}\right\}^{(1)}=\left\{A_{2} \omega^{3}-C_{3} \bar{\theta}, A_{2} \omega^{4}-C_{4} \bar{\theta}\right\},
\end{aligned}
$$

and that each of these derived systems is integrable.

3.1.2. Case 1.2: Exactly one of $\mathcal{K}_{1}^{(\infty)}$ and $\mathcal{K}_{2}^{(\infty)}$ has rank 3. Without loss of generality, we may assume that $\mathcal{K}_{2}^{(\infty)}$ has rank 2 and is equal to $\{d Y, d Q\}$, and that $\mathcal{K}_{1}^{(\infty)}$ has rank 3. It follows that (3.5) vanishes identically and that (3.6) does 
not. Since all our results are local, we will assume that we are working on an open set where (3.6) is nonzero. The vanishing of (3.5) implies that

$$
A_{2,3}=-A_{2} C_{4}\left(A_{2}-1\right), \quad A_{2,4}=A_{2} C_{3}\left(A_{2}-1\right) .
$$

Recall that the function $\frac{C_{3}}{C_{4}}$ satisfies

$$
d\left(\frac{C_{3}}{C_{4}}\right) \in \mathcal{K}_{2}^{(\infty)}=\{d Y, d Q\} .
$$

It follows that $\frac{C_{3}}{C_{4}}$ is a function of $Y$ and $Q$ alone. Now consider the 1-form

$$
\widetilde{\omega}^{3}=\omega^{3}-\frac{C_{3}}{C_{4}} \omega^{4}=d Y-\frac{C_{3}}{C_{4}} d Q .
$$

This 1-form is contained in the span of $\omega^{3}, \omega^{4}$, and we have

$$
d \widetilde{\omega}^{3} \equiv 0 \quad \bmod \widetilde{\omega}^{3}
$$

so $\widetilde{\omega}^{3}$ is a multiple of an exact 1 -form, say $\widetilde{\omega}^{3}=\lambda d \widetilde{Y}$. Moreover, because $\widetilde{\omega}^{3}$ is expressed solely in terms of $Y$ and $Q, \lambda$ and $\tilde{Y}$ may be chosen to be functions depending only on $Y$ and $Q$, and which are therefore well-defined on $\mathcal{M}$. The crucial point here is that there exists an exact 1-form in the span of $\left\{\omega^{3}, \omega^{4}\right\}$ which is well-defined on $\mathcal{M}$. Then we have

$$
d Y \wedge d Q=\widetilde{\omega}^{3} \wedge d Q=\lambda d \widetilde{Y} \wedge d Q=d \widetilde{Y} \wedge d \widetilde{Q}
$$

where

$$
\widetilde{Q}(\widetilde{Y}, Q)=\int_{0}^{Q} \lambda(\widetilde{Y}, t) d t
$$

Since

$$
d \theta=d X \wedge d P+d \widetilde{Y} \wedge d \widetilde{Q},
$$

Pfaff's Theorem (see Ch. 1 of [8]) implies that there exists a function $\widetilde{Z}$ on $\mathcal{M}$ such that

$$
\theta=d \widetilde{Z}-P d X-\widetilde{Q} d \widetilde{Y}
$$

We can now repeat all our constructions starting with the coordinate system $(X, \widetilde{Y}, \widetilde{Z}, P, \widetilde{Q})$, but now our adapted coframing $\sigma$ will have the additional property that $\omega^{3}=d \widetilde{Y}$ and $C_{3}=0$. Thus we will drop the tildes and assume that $C_{3}=0$ for the remainder of this subsection.

Proposition 3.2. If $\left[\begin{array}{ll}C_{1} & C_{2}\end{array}\right],\left[\begin{array}{ll}C_{3} & C_{4}\end{array}\right]$ are both nonzero, $\mathcal{K}_{1}^{(\infty)}$ has rank 3, and $\mathcal{K}_{2}^{(\infty)}$ has rank 2, then the system $(\overline{\mathcal{M}}, \overline{\mathcal{I}})$ is Monge-integrable, and it becomes Darboux-integrable after one prolongation.

Proof. The same argument as that given in Case 1.1 shows that the characteristic system $\left\{\bar{\theta}, \omega^{1}, \omega^{2}\right\}$ on $\overline{\mathcal{M}}$ contains a rank 2 integrable subsystem; therefore, $(\overline{\mathcal{M}}, \overline{\mathcal{I}})$ is Monge-integrable. 
In order to prove the second half of the Proposition, we will need to make use of relations among the second derivatives of the functions $A_{2}, C_{1}, C_{2}, C_{4}$. These will be denoted as, e.g.,

$$
d A_{2,0}=A_{2,00} \theta+A_{2,00} \bar{\theta}+A_{2,01} \omega^{1}+A_{2,02} \omega^{2}+A_{2,03} \omega^{3}+A_{2,04} \omega^{4} .
$$

Note that, although (for example) the $A_{2, i j}$ are second derivatives of $A_{2}$, because we are working in a coframing rather than in coordinates, we cannot assume that $A_{2, i j}=A_{2, j i}$.

Computing $d\left(d A_{2}\right) \equiv 0 \bmod \left\{\bar{\theta}, \omega^{3}, \omega^{4}\right\}$ shows that

$$
\begin{array}{r}
A_{2,10}=A_{2}\left(C_{4,41}-C_{1,11}-C_{2,21}+C_{2}\left(C_{1,1}+C_{2,2}-C_{4,4}\right)\right) \\
\quad+A_{2,1}\left(C_{4,4}-C_{2,2}-C_{1} C_{2}\right)+A_{2,2}\left(C_{2,1}-C_{2}^{2}\right), \\
A_{2,20}=A_{2}\left(C_{4,42}-C_{1,12}-C_{2,22}+C_{1}\left(C_{4,4}-C_{1,1}-C_{2,2}\right)\right) \\
\quad+A_{2,1}\left(C_{1,2}+C_{1}^{2}\right)+A_{2,2}\left(C_{4,4}-C_{1,1}+C_{1} C_{2}\right), \\
A_{2,21}=A_{2,12}+\left(A_{2}-1\right)\left(C_{1,1}+C_{2,2}-\frac{A_{2,1}}{A_{2}} C_{1}-\frac{A_{2,2}}{A_{2}} C_{2}\right) .
\end{array}
$$

Next, computing $d\left(d C_{4}\right) \equiv 0 \bmod \{\theta, \bar{\theta}\}$ shows that

$$
\begin{aligned}
& C_{4,34}=C_{4,43}+\left(A_{2}-1\right) C_{4, \overline{0}}+C_{4}\left(C_{1,1}+C_{2,2}\right), \\
& C_{4,31}=C_{2}\left(C_{4,3}+C_{4}^{2}\right), \\
& C_{4,32}=-C_{1}\left(C_{4,3}+C_{4}^{2}\right), \\
& C_{4,41}=C_{2} C_{4,4} \\
& C_{4,42}=-C_{1} C_{4,4} .
\end{aligned}
$$

Now computing $d\left(d C_{4}\right) \equiv 0 \bmod \theta$ shows that

$$
\begin{aligned}
& C_{4, \overline{0} 1}=C_{4} C_{2, \overline{0}}+\frac{\left(\left(A_{2}+1\right) C_{1}-A_{2,1}\right)}{A_{2}} C_{4, \overline{0}}, \\
& C_{4, \overline{0} 2}=-C_{4} C_{1, \overline{0}}-\frac{\left(\left(A_{2}+1\right) C_{2}+A_{2,2}\right)}{A_{2}} C_{4, \overline{0}}, \\
& C_{4,3 \overline{0}}=C_{4, \overline{0} 3}-A_{2} C_{4} C_{4, \overline{0}}, \\
& C_{4,4 \overline{0}}=C_{4, \overline{0} 4},
\end{aligned}
$$

and then computing $d\left(d C_{4}\right) \equiv 0 \bmod \bar{\theta}$ shows that

$$
\begin{aligned}
& C_{4,30}=C_{4}\left(C_{1,13}+C_{2,23}-C_{4,43}+C_{4, \overline{0}}\right)+\left(C_{4,3}-C_{4}^{2}\right)\left(C_{1,1}+C_{2,2}\right)-C_{4, \overline{0} 3}, \\
& C_{4,40}=C_{4}\left(C_{1,14}+C_{2,24}-C_{4,44}\right)+C_{4,4}\left(C_{1,1}+C_{2,2}\right)-C_{4, \overline{0} 4}, \\
& C_{1,12}=-C_{2,22}+\left(A_{2}-1\right) C_{1, \overline{0}}-C_{1} C_{4,4}+\frac{\left(\left(A_{2}-1\right) C_{1}-A_{2,2}\right)}{A_{2} C_{4}} C_{4, \overline{0}} \\
& C_{2,21}=-C_{1,11}-\left(A_{2}-1\right) C_{2, \overline{0}}+C_{2} C_{4,4}-\frac{\left(\left(A_{2}-1\right) C_{2}+A_{2,1}\right)}{A_{2} C_{4}} C_{4, \overline{0}} .
\end{aligned}
$$


Next, computing $d\left(d C_{1}\right) \equiv 0 \bmod \{\theta, \bar{\theta}\}$ yields

$$
\begin{aligned}
& C_{1,13}=C_{4}\left(C_{1,1}+C_{1} C_{2}\right), \\
& C_{1,14}=0 \\
& C_{1,23}=C_{4}\left(C_{1,2}-C_{1}^{2}\right), \\
& C_{1,24}=0 \\
& C_{1,21}=-C_{2,22}-2 C_{1} C_{4,4}+2\left(A_{2}-1\right) C_{1, \overline{0}}+\frac{\left(\left(A_{2}-1\right) C_{1}-A_{2,2}\right)}{A_{2} C_{4}} C_{4, \overline{0}} .
\end{aligned}
$$

Similarly, computing $d\left(d C_{2}\right) \equiv 0 \bmod \{\theta, \bar{\theta}\}$ yields

$$
\begin{aligned}
& C_{2,13}=C_{4}\left(C_{42,1}+C_{2}^{2}\right), \\
& C_{2,14}=0 \\
& C_{2,23}=C_{4}\left(C_{2,2}-C_{1} C_{2}\right), \\
& C_{2,24}=0 \\
& C_{2,12}=-C_{1,11}+2 C_{2} C_{4,4}-2\left(A_{2}-1\right) C_{2, \overline{0}}-\frac{\left(\left(A_{2}-1\right) C_{2}+A_{2,1}\right)}{A_{2} C_{4}} C_{4, \overline{0}} .
\end{aligned}
$$

Computing $d\left(d C_{1}\right) \equiv 0 \bmod \left\{\omega^{2}, \omega^{3}, \omega^{4}\right\}$ yields

$$
\begin{aligned}
C_{1,10}=C_{2} C_{2,22}-C_{1} C_{1,11}-A_{2} C_{1, \overline{0} 1}+\left(\left(2-A_{2}\right) C_{2}-A_{2,1}\right) C_{1, \overline{0}}+C_{4,4}\left(C_{1,1}+2 C_{1} C_{2}\right) & \\
& -\frac{\left(\left(A_{2}-1\right) C_{1}-A_{2,2}\right)}{A_{2} C_{4}} C_{2} C_{4, \overline{0}}, \\
C_{1,1 \overline{0}}=C_{1, \overline{0} 1}+ & \frac{\left(A_{2,1}-C_{2}\right)}{A_{2}} C_{1, \overline{0}},
\end{aligned}
$$

and computing $d\left(d C_{2}\right) \equiv 0 \bmod \left\{\omega^{1}, \omega^{3}, \omega^{4}\right\}$ yields

$$
\begin{aligned}
C_{2,20}= & C_{1} C_{1,11}-C_{2} C_{2,22}-A_{2} C_{2, \overline{0} 2}+\left(\left(A_{2}-2\right) C_{1}-A_{2,2}\right) C_{2, \overline{0}}+C_{4,4}\left(C_{2,2}-2 C_{1} C_{2}\right) \\
& +\frac{\left(\left(A_{2}-1\right) C_{2}+A_{2,1}\right)}{A_{2} C_{4}} C_{1} C_{4, \overline{0}}, \\
C_{2,2 \overline{0}}=C_{2, \overline{0} 2}+ & \frac{\left(A_{2,2}+C_{1}\right)}{A_{2}} C_{2, \overline{0}},
\end{aligned}
$$

Now computing $d\left(d A_{2}\right) \equiv 0 \bmod \omega^{3}$ yields

$$
\begin{aligned}
A_{2,1 \overline{0}}=\frac{1}{A_{2}}[ & \left.-C_{1} A_{2,11}-C_{2} A_{2,12}+A_{2,1}\left(C_{1} C_{2}+C_{2,2}\right)+A_{2,2}\left(-C_{2,1}+C_{2}^{2}\right)\right] \\
& -\left(A_{2}-1\right) C_{2, \overline{0}}-C_{2}\left(C_{1,1}+C_{2,2}\right)-\left(\left(A_{2}-2\right) C_{2}+2 A_{2,1}\right) C_{4,4} \\
& -\frac{\left(\left(A_{2}-1\right) C_{2}+A_{2,1}\right)}{A_{2} C_{4}} C_{4, \overline{0}} \\
A_{2,2 \overline{0}}=\frac{1}{A_{2}}[- & \left.C_{1} A_{2,12}-C_{2} A_{2,22}-A_{2,1}\left(C_{1,2}+\frac{C_{1}^{2}}{A_{2}}\right)+A_{2,2}\left(C_{1,1}-\frac{C_{1} C_{2}}{A_{2}}\right)\right] \\
& +\left(A_{2}-1\right) C_{1, \overline{0}}+\frac{C_{1}}{A_{2}}\left(C_{1,1}+C_{2,2}\right)+\left(\left(A_{2}-2\right) C_{1}-2 A_{2,2}\right) C_{4,4} \\
& +\frac{\left(\left(A_{2}-1\right) C_{1}-A_{2,2}\right)}{A_{2} C_{4}} C_{4, \overline{0}}
\end{aligned}
$$


and

$$
A_{2,14}=A_{2,24}=C_{4,44}=0 .
$$

Finally, we need two additional relations which do not become apparent until we differentiate the equations for $d C_{4,3}$ and $d C_{4,4}$. Computing $d\left(d C_{4,3}\right) \equiv 0 \bmod \{\bar{\theta}-$ $\left.\theta, \omega^{3}, \omega^{4}-C_{4} \theta\right\}$ yields

$$
\left(C_{4} C_{4, \overline{0} 3}-\left(\left(A_{2}+1\right) C_{4}^{2}+C_{4,3}\right) C_{4, \overline{0}}\right) \theta \wedge\left[\left(A_{2,1}+C_{2}\left(A_{2}-1\right)\right) \omega^{1}+\left(A_{2,2}-C_{1}\left(A_{2}-1\right)\right) \omega^{2}\right] .
$$

Note that the right-hand factor is precisely (3.6), which we have assumed is nonzero. Therefore,

$$
C_{4, \overline{0} 3}=\frac{C_{4, \overline{0}}\left(C_{4,3}+\left(A_{2}+1\right) C_{4}^{2}\right)}{C_{4}}
$$

Precisely the same argument applied to $d\left(d C_{4,4}\right)$ shows that

$$
C_{4, \overline{0} 4}=\frac{C_{4, \overline{0}} C_{4,4}}{C_{4}}
$$

With these relations in hand, consider the characteristic system $\overline{\mathcal{K}}=\left\{\bar{\theta}, \omega^{3}, \omega^{4}\right\}$ of $\overline{\mathcal{I}}$ - which is well-defined on $\overline{\mathcal{M}}$, even though $\omega^{4}$ is not. We need to show that after one prolongation, the corresponding characteristic system $\overline{\mathcal{K}}^{\prime}$ of the prolongation contains a rank 2 integrable subsystem. In order to perform this computation, we need to construct a basis for $\overline{\mathcal{K}}$ consisting of 1 -forms which are well-defined on $\overline{\mathcal{M}}$. Fortunately, $\bar{\theta}$ and $\omega^{3}$ are already well-defined on $\overline{\mathcal{M}}$. For the remaining 1-form, it will be convenient to choose a 1 -form which is contained in the first derived system $\overline{\mathcal{K}}^{(1)}=\left\{\omega^{4}-C_{4} \bar{\theta}, \omega^{3}\right\}$. To this end, introduce functions $\tau, g$ on $\mathcal{B}$ such that the 1 -form

$$
\psi=e^{\tau}\left(\omega^{4}-C_{4} \bar{\theta}-g \omega^{3}\right)
$$

is well-defined on $\overline{\mathcal{M}}$. (The fact that $\overline{\mathcal{K}}^{(1)}$ is well-defined on $\overline{\mathcal{M}}$ guarantees the existence of such functions.) As before, we denote the derivatives of these functions by

$$
\begin{aligned}
& d \tau=\tau_{0} \theta+\tau_{\overline{0}} \bar{\theta}+\tau_{1} \omega^{1}+\tau_{2} \omega^{2}+\tau_{3} \omega^{3}+\tau_{4} \omega^{4}, \\
& d g=g_{0} \theta+g_{\overline{0}} \bar{\theta}+g_{1} \omega^{1}+g_{2} \omega^{2}+g_{3} \omega^{3}+g_{4} \omega^{4},
\end{aligned}
$$

and similarly for second derivatives.

Because $\psi$ is well-defined on $\overline{\mathcal{M}}, d \psi$ contains no terms involving $\theta$. This, in turn, determines the partial derivatives $\tau_{0}, g_{0}$ :

$$
\begin{aligned}
\tau_{0} & =C_{4,4}, \\
g_{0} & =C_{4}^{2}-C_{4,3}-g C_{4,4} .
\end{aligned}
$$

We will also need to make use of relations among the second derivatives of $\tau, g$. These are determined by computing $d(d \tau)=d(d g)=0$; this is a straightforward computation, which we omit here for the sake of brevity.

We can define a partial prolongation $\overline{\mathcal{I}}^{\prime}$ of $\overline{\mathcal{I}}$ on $\overline{\mathcal{M}} \times \mathbb{R}$ as follows. (Note that Darboux-integrability of the partial prolongation implies Darboux-integrability of the 
full prolongation.) Let $t$ be a new coordinate on the $\mathbb{R}$ factor; then the partial prolongation $\overline{\mathcal{I}}^{\prime}$ is generated by the 1 -forms $\bar{\theta}, \bar{\theta}^{\prime}=\psi-t \omega^{3}$, and the 2 -form $\omega^{1} \wedge \omega^{2}$. Again, this system is well-defined on $\overline{\mathcal{M}} \times \mathbb{R}$, even though $\omega^{1}, \omega^{2}$ are not.

A straightforward computation shows that

$$
d \bar{\theta}^{\prime} \equiv-\pi_{1} \wedge \omega^{3} \bmod \left\{\bar{\theta}, \bar{\theta}^{\prime}\right\}
$$

where

$$
\pi_{1}=d t-\left(t \tau_{1}-e^{\tau} g_{1}\right) \omega^{1}-\left(t \tau_{2}-e^{\tau} g_{2}\right) \omega^{2} .
$$

The corresponding characteristic system of $\overline{\mathcal{I}}^{\prime}$ is

$$
\overline{\mathcal{K}}^{\prime}=\left\{\bar{\theta}, \bar{\theta}^{\prime}, \pi_{1}, \omega^{3}\right\} .
$$

We will now compute the derived systems of $\overline{\mathcal{K}}^{\prime}$ and show that the second derived system $\overline{\mathcal{K}}^{\prime(2)}$ is a Frobenius system of rank 2; this will complete the proof of the Proposition. In order to compute the first derived system, we compute:

$$
\left.\begin{array}{rl}
d \bar{\theta} & \equiv \omega^{1} \wedge \omega^{2} \\
d \bar{\theta}^{\prime} & \equiv 0 \\
d \pi_{1} & \equiv E \omega^{1} \wedge \omega^{2} \\
d \omega^{3} & \equiv 0
\end{array}\right\} \quad \bmod \left\{\bar{\theta}, \bar{\theta}^{\prime}, \pi_{1}, \omega^{3}\right\}
$$

(the last line following from $C_{3}=0$ ), where

$$
E=e^{\tau} C_{4}^{2}+\left(e^{\tau} g_{4}-t \tau_{4}\right) C_{4}-e^{\tau} C_{4,3}-\left(e^{\tau} g+t\right) C_{4,4}+\left(e^{\tau} g_{\overline{0}}-t \tau_{\overline{0}}\right) .
$$

Let $\pi_{2}=\pi_{1}-E \bar{\theta}$, so that

$$
\overline{\mathcal{K}}^{\prime(1)}=\left\{\bar{\theta}^{\prime}, \pi_{2}, \omega^{3}\right\} .
$$

Next we compute the derived system of $\overline{\mathcal{K}}^{\prime(1)}$ :

$$
\left.\begin{array}{rl}
d \bar{\theta}^{\prime} & \equiv \frac{C_{4}}{A_{2}} \bar{\theta} \wedge\left[\left(A_{2,1}+C_{2}\left(A_{2}-1\right)\right) \omega^{1}+\left(A_{2,2}-C_{1}\left(A_{2}-1\right)\right) \omega^{2}\right] \\
d \pi_{2} & \equiv \frac{F}{A_{2}} \bar{\theta} \wedge\left[\left(A_{2,1}+C_{2}\left(A_{2}-1\right)\right) \omega^{1}+\left(A_{2,2}-C_{1}\left(A_{2}-1\right)\right) \omega^{2}\right] \\
d \omega^{3} & \equiv 0
\end{array}\right\} \bmod \left\{\bar{\theta}^{\prime}, \pi_{2}, \omega^{3}\right\},
$$

where

$$
F=e^{\tau} C_{4}^{2}+\left(e^{\tau} g_{4}-t \tau_{4}\right) C_{4}-e^{\tau} C_{4,3}-\left(e^{\tau} g+t\right) C_{4,4} .
$$

Once again, we see the bracketed 1-form in (3.6) appearing. Since this 1-form is assumed to be nonzero, the derived system $\overline{\mathcal{K}}^{(2)}$ has rank 2 and is spanned by the forms $\omega^{3}$ and

$$
\pi_{3}=C_{4} \pi_{2}-F \bar{\theta}
$$


Finally, another computation shows that

$$
\left.\begin{array}{l}
d \pi_{3} \equiv 0 \\
d \omega^{3} \equiv 0
\end{array}\right\} \quad \bmod \left\{\pi_{3}, \omega^{3}\right\}
$$

therefore, $\overline{\mathcal{K}}^{\prime(2)}$ is integrable.

3.1.3. Case 1.3: $\mathcal{K}_{1}^{(\infty)}$ and $\mathcal{K}_{2}^{(\infty)}$ both have rank 2. Now we assume that the bracketed 1-forms in both (3.5) and (3.6) are nonzero. By the same argument as that given in the previous case, we may assume that $C_{1}=C_{3}=0$, with $\omega^{1}=d X, \omega^{3}=d Y$.

Proposition 3.3. If $\left[\begin{array}{ll}C_{1} & C_{2}\end{array}\right],\left[\begin{array}{ll}C_{3} & C_{4}\end{array}\right]$ are both nonzero and $\mathcal{K}_{1}^{(\infty)}$ and $\mathcal{K}_{2}^{(\infty)}$ both have rank 2, then the system $(\overline{\mathcal{M}}, \overline{\mathcal{I}})$ becomes Darboux-integrable after one prolongation.

Proof. The proof is very similar to that of Proposition 3.2. We must now consider both characteristic systems

$$
\overline{\mathcal{K}}_{1}=\left\{\bar{\theta}, \omega^{1}, \omega^{2}\right\}, \quad \overline{\mathcal{K}}_{2}=\left\{\bar{\theta}, \omega^{3}, \omega^{4}\right\}
$$

of $\overline{\mathcal{I}}$. As before, these systems are both well-defined on $\overline{\mathcal{M}}$, even though $\omega^{2}$ and $\omega^{4}$ are not. We introduce functions $\rho, \tau, f, g$ on $\mathcal{B}$ such that the 1 -forms

$$
\eta=e^{\rho}\left(A_{2} \omega^{2}-C_{2} \bar{\theta}-f \omega^{1}\right), \quad \psi=e^{\tau}\left(\omega^{4}-C_{4} \bar{\theta}-g \omega^{3}\right)
$$

are well-defined on $\overline{\mathcal{M}}$. These forms have the property that

$$
\overline{\mathcal{K}}_{1}^{(1)}=\left\{\eta, \omega^{1}\right\}, \quad \overline{\mathcal{K}}_{2}^{(1)}=\left\{\psi, \omega^{3}\right\}
$$

We construct the prolongation $\overline{\mathcal{I}}^{\prime}$ of $\overline{\mathcal{I}}$ on $\overline{\mathcal{M}} \times \mathbb{R}^{2}$ as follows. Let $r, t$ be new coordinates on the $\mathbb{R}^{2}$ factor; then the prolongation $\overline{\mathcal{I}}^{\prime}$ is generated by the 1 -forms $\bar{\theta}, \bar{\theta}_{1}=\eta-r \omega^{1}, \bar{\theta}_{2}=\psi-t \omega^{3}$, and their exterior derivatives.

The remainder of the proof consists of applying the argument of Proposition 3.2 to each of the characteristic systems $\overline{\mathcal{K}}_{1}^{\prime}, \overline{\mathcal{K}}_{2}^{\prime}$ of the prolongation $\overline{\mathcal{I}}^{\prime}$. The argument varies only in the details of the calculations, and so we omit it for the sake of brevity.

3.2. Case 2: One of the $C$-vectors vanishes. Without loss of generality, assume that $\left[\begin{array}{ll}C_{1} & C_{2}\end{array}\right]=\left[\begin{array}{ll}0 & 0\end{array}\right]$, and that $C_{4} \neq 0$. By Proposition 2.3, it follows that $\left[\begin{array}{ll}B_{1} & B_{2}\end{array}\right]=\left[\begin{array}{ll}0 & 0\end{array}\right]$ as well.

Proposition 3.4. If $\left[\begin{array}{ll}C_{1} & C_{2}\end{array}\right]=\left[\begin{array}{ll}0 & 0\end{array}\right]$, then the system $(\overline{\mathcal{M}}, \overline{\mathcal{I}})$ is Mongeintegrable, and it becomes Darboux-integrable after at most one prolongation. Furthermore, the Bäcklund transformation $\mathcal{B} \subset \mathcal{M} \times \overline{\mathcal{M}}$ is holonomic.

Proof. It follows from Proposition 2.3 that $(\overline{\mathcal{M}}, \overline{\mathcal{I}})$ is Monge-integrable; in fact, the characteristic system $\left\{\bar{\theta}, \omega^{1}, \omega^{2}\right\}$ contains $\left\{\omega^{1}, \omega^{2}\right\}=\{d X, d P\}$ as a rank 2 integrable subsystem.

Now consider the other characteristic system $\mathcal{K}=\left\{\bar{\theta}, \omega^{3}, \omega^{4}\right\}$ of $\overline{\mathcal{I}}$. One easily computes that the first derived system of $\mathcal{K}$ is

$$
\mathcal{K}^{(1)}=\left\{\omega^{3}-C_{3} \bar{\theta}, \omega^{4}-C_{4} \bar{\theta}\right\}
$$


In order to find the second derived system, we compute:

$$
\left.\begin{array}{rl}
d\left(\omega^{3}-C_{3} \bar{\theta}\right) & \equiv \frac{C_{3}}{A_{2}} \bar{\theta} \wedge\left(A_{2,1} \omega^{1}+A_{2,2} \omega^{2}\right) \\
d\left(\omega^{4}-C_{4} \bar{\theta}\right) & \equiv \frac{C_{4}}{A_{2}} \bar{\theta} \wedge\left(A_{2,1} \omega^{1}+A_{2,2} \omega^{2}\right)
\end{array}\right\} \quad \bmod \mathcal{K}^{(1)} .
$$

If $A_{2,1}=A_{2,2}=0$, then $\mathcal{K}^{(1)}$ is integrable; in this case, $\overline{\mathcal{I}}$ is Darboux-integrable and hence contact equivalent to the standard wave equation. Therefore, we assume that $A_{2,1}$ and $A_{2,2}$ are not both zero.

In order to prove the second statement, we will construct a partial prolongation of $\overline{\mathcal{I}}$ and proceed as in $\S 3.1 .2$. But first we need to derive relations among the derivatives of the torsion functions.

Picking up where we left off at (3.4), consider $d\left(d C_{3}\right), d\left(d C_{4}\right)$. Computing $d\left(d C_{3}\right) \equiv d\left(d C_{4}\right) \equiv 0 \bmod \left\{\theta-\bar{\theta}, \omega^{3}-C_{3} \bar{\theta}, \omega^{4}-C_{4} \bar{\theta}\right\}$ yields

$$
C_{3, \overline{0}}=C_{4, \overline{0}}=0
$$

From (3.3), it follows that $B_{3}=B_{4}=0$; therefore, the Bäcklund transformation is holonomic, as claimed.

We now have

$$
\begin{aligned}
& d C_{3}=C_{3,3}\left(\omega^{3}-C_{3} \theta\right)+C_{3,4}\left(\omega^{4}-C_{4} \theta\right)=C_{3,3} d Y+C_{3,4} d Q, \\
& d C_{4}=C_{4,3}\left(\omega^{3}-C_{3} \theta\right)+C_{4,4}\left(\omega^{4}-C_{4} \theta\right)=C_{4,3} d Y+C_{4,4} d Q .
\end{aligned}
$$

It follows that $C_{3}, C_{4}$ are functions of $Y$ and $Q$ alone. Now the same argument as that given in $\S 3.1 .2$ shows that we may assume $C_{3}=0$; moreover, $C_{4}$ remains a function of $Y$ and $Q$ alone when we do so. Computing $d\left(d C_{4}\right)=0$ yields the following relations among the second derivatives of $C_{4}$ :

$$
\begin{gathered}
C_{4,30}=-C_{4} C_{4,34}, \quad C_{4,40}=-C_{4} C_{4,44}, \quad C_{4,43}=C_{4,34}, \\
C_{4,3 \overline{0}}=C_{4,4 \overline{0}}=C_{4,31}=C_{4,32}=C_{4,41}=C_{4,42}=0 .
\end{gathered}
$$

Now consider the characteristic system $\mathcal{K}=\left\{\bar{\theta}, \omega^{3}, \omega^{4}\right\}$. As we computed above (recalling that $C_{3}=0$ ), its first derived system is

$$
\mathcal{K}^{(1)}=\left\{\omega^{3}, \omega^{4}-C_{4} \bar{\theta}\right\} .
$$

As in $\S 3.1 .2$, choose functions $g, \tau$ so that the 1 -form

$$
\psi=e^{\tau}\left(\omega^{4}-C_{4} \bar{\theta}-g \omega^{3}\right)
$$

is well-defined on $\overline{\mathcal{M}}$, and construct the partial prolongation $\overline{\mathcal{I}}^{\prime}$ of $\overline{\mathcal{I}}$ and the 1-form $\bar{\theta}^{\prime}$ as we did there. Similar calculations to those of $\S 3.1 .2$ show that the corresponding characteristic system $\overline{\mathcal{K}}^{\prime}$ of $\overline{\mathcal{I}}^{\prime}$ has a rank 2 integrable subsystem. This completes the proof. $\mathrm{Q}$ 


\section{Proof that Darboux implies Bäcklund.}

4.1. The non-Monge-integrable case. In this subsection $(\mathcal{M}, \mathcal{I})$ is assumed to be a hyperbolic Monge-Ampère system which is Darboux-integrable after one prolongation, but not Monge-integrable. We will construct a canonical coframing associated to the prolongation. We will then use this coframing to construct an integrable extension $\mathcal{J}$ of $(\mathcal{M}, \mathcal{I})$ in such a way that $\mathcal{J}$ defines a Bäcklund transformation between $(\mathcal{M}, \mathcal{I})$ and the standard wave equation $Z_{X Y}=0$.

LEMma 4.1. Near any point of $\mathcal{M}$, there exists a coframing $\left(\theta, \pi_{1}, \pi_{2}, \eta^{1}, \eta^{2}\right)$ such that $\theta$ spans the 1-forms of $\mathcal{I}$, and the characteristic systems $\mathcal{C}_{1}, \mathcal{C}_{2}$ of $\mathcal{I}$ have derived flags

$$
\begin{aligned}
& \mathcal{C}_{1}=\left\{\theta, \pi_{1}, \eta^{1}\right\} \supset\left\{\pi_{1}, \eta^{1}\right\} \supset\left\{\eta^{1}\right\}=\mathcal{C}_{1}^{(\infty)} \\
& \mathcal{C}_{2}=\left\{\theta, \pi_{2}, \eta^{2}\right\} \supset\left\{\pi_{2}, \eta^{2}\right\} \supset\left\{\eta^{2}\right\}=\mathcal{C}_{2}^{(\infty)}
\end{aligned}
$$

Proof. By a result of Juráš [10], $(\mathcal{M}, \mathcal{I})$ is locally contact equivalent to a system encoding a PDE of the form

$$
u_{x y}=F(x, y, u, p, q) .
$$

Thus, there are local coordinates $x, y, u, p, q$ near the given point of $\mathcal{N}$ such that $\mathcal{I}$ is generated by the 1-form $\theta=d u-p d x-q d y$ and the 2 -forms $(d p-F d y) \wedge d x$ and $(d q-F d x) \wedge d y$. It is easy to verify that the coframing given by $\theta, \eta^{1}=d x, \eta^{2}=d y$, $\pi_{1}=d p-F d y-F_{q} \theta$, and $\pi_{2}=d q-F d x-F_{p} \theta$ has the properties claimed.

In terms of the local coframing on $\mathcal{M}$ given by the lemma, the prolongation $\left(\mathcal{M}^{\prime}, \mathcal{I}^{\prime}\right)$ is defined as follows: let $\mathcal{M}^{\prime}=\mathcal{M} \times \mathbb{R}^{2}$, with coordinates $r, t$ on the $\mathbb{R}^{2}$ factor, and let $\mathcal{I}^{\prime}$ be the Pfaffian system on $\mathcal{M}^{\prime}$ generated by $\theta$ and the forms

$$
\theta_{1}=\pi_{1}-r \eta^{1}, \quad \theta_{2}=\pi_{2}-t \eta^{2} .
$$

Lemma 4.2. Near any point of $\mathcal{M}^{\prime}$ there exists a coframing $\left(\theta, \theta_{1}, \theta_{2}, \eta^{1}, \eta^{2}, \pi_{3}, \pi_{4}\right)$ such that $\mathcal{I}^{\prime}$ is generated by $\theta, \theta_{1}, \theta_{2}$, satisfying

$$
\begin{aligned}
d \theta & =-\theta_{1} \wedge \eta^{1}-\theta_{2} \wedge \eta^{2} \quad \bmod \theta \\
d \theta_{1} & =-\pi_{3} \wedge \eta^{1} \quad \bmod \theta, \theta_{1} \\
d \theta_{2} & =-\pi_{4} \wedge \eta^{2} \quad \bmod \theta, \theta_{2},
\end{aligned}
$$

with the derived flags of the characteristic systems of $\mathcal{I}^{\prime}$ given by

$$
\begin{aligned}
& \mathcal{C}_{1}^{\prime}=\left\{\theta, \theta_{1}, \theta_{2}, \eta^{1}, \pi_{3}\right\} \supset\left\{\theta, \theta_{1}, \eta^{1}, \pi_{3}\right\} \supset\left\{\theta_{1}, \eta^{1}, \pi_{3}\right\} \supset\left\{\eta^{1}, \pi_{3}\right\}=\mathcal{C}_{1}^{\prime(\infty)}, \\
& \mathcal{C}_{2}^{\prime}=\left\{\theta, \theta_{1}, \theta_{2}, \eta^{2}, \pi_{4}\right\} \supset\left\{\theta, \theta_{2}, \eta^{2}, \pi_{4}\right\} \supset\left\{\theta_{2}, \eta^{2}, \pi_{4}\right\} \supset\left\{\eta^{2}, \pi_{4}\right\}=\mathcal{C}_{2}^{\prime(\infty)} .
\end{aligned}
$$

Proof. Let $\theta, \eta^{1}=d x, \eta^{2}=d y$ be part of the local coframing on $\mathcal{M}$ (pulled back to $\left.\mathcal{M}^{\prime}\right)$ constructed in the proof of Lemma 4.1, and let $\theta_{1}, \theta_{2}$ be defined as in (4.1). Then

$$
\begin{array}{ll}
d \theta_{1} \equiv-\left(d r-\left(D_{x} F\right) d y\right) \wedge d x & \bmod \theta, \theta_{1}, \\
d \theta_{2} \equiv-\left(d t-\left(D_{y} F\right) d x\right) \wedge d y & \bmod \theta, \theta_{2},
\end{array}
$$


where

$$
\begin{aligned}
& D_{x} F=F_{x}+F_{u} p+F_{p} r+F_{q} F, \\
& D_{y} F=F_{y}+F_{u} q+F_{p} F+F_{q} t .
\end{aligned}
$$

For the moment, let $\pi_{3}=d r-\left(D_{x} F\right) d y$. Because $d \pi_{3} \equiv 0$ modulo $d x, \pi_{3}, \theta, \theta_{1}, \theta_{2}$, it follows that $\pi_{3}$ lies in $\mathcal{C}_{1}^{(1)}$. Moreover, we may subtract a multiple of $\theta$ from $\pi_{3}$ to ensure that $\pi_{3}$ lies in $\mathcal{C}_{1}^{\prime(2)}$.

Next, we prove that the last derived system of $\mathcal{C}_{1}^{\prime}$ has rank 2 , rather than rank 3. (A similar argument applies to $\mathcal{C}_{2}^{\prime}$.) Suppose that $\mathcal{C}_{1}^{\prime(2)}=\left\{\theta_{1}, \eta^{1}, \pi_{3}\right\}$ is integrable. From (4.1), it is clear that this is equivalent to the statement that $\left\{\pi_{1}, \eta^{1}, \pi_{3}\right\}$ is integrable -i.e., that $d \pi_{1} \equiv d \eta^{1} \equiv d \pi_{3} \equiv 0$ modulo $\pi_{1}, \eta^{1}, \pi_{3}$. But $\pi_{1}$ and $\eta^{1}$ are both well-defined on $\mathcal{M}$, so their exterior derivatives do not involve $\pi_{3}$. It follows that $d \pi_{1} \equiv d \eta^{1} \equiv 0$ modulo $\pi_{1}, \eta^{1}$, and $\mathcal{C}_{1}^{(1)}=\left\{\pi_{1}, \eta^{1}\right\}$ is integrable, contrary to the hypothesis that $(\mathcal{M}, \mathcal{I})$ is not Monge-integrable.

The conditions in Lemma 4.2 are preserved by changes of coframing of the form

$$
\left[\begin{array}{c}
\tilde{\theta} \\
\tilde{\theta}_{1} \\
\tilde{\theta}_{2} \\
\tilde{\eta}^{1} \\
\tilde{\eta}^{2} \\
\tilde{\pi}_{3} \\
\tilde{\pi}_{4}
\end{array}\right]=\left[\begin{array}{ccccccc}
c & 0 & 0 & 0 & 0 & 0 & 0 \\
0 & a_{1} c & 0 & 0 & 0 & 0 & 0 \\
0 & 0 & a_{2} c & 0 & 0 & 0 & 0 \\
0 & 0 & 0 & a_{1}^{-1} & 0 & 0 & 0 \\
0 & 0 & 0 & 0 & a_{2}^{-1} & 0 & 0 \\
0 & 0 & 0 & b_{1} & 0 & a_{1}^{2} c & 0 \\
0 & 0 & 0 & 0 & b_{2} & 0 & a_{2}^{2} c
\end{array}\right]^{-1}\left[\begin{array}{c}
\theta \\
\theta_{1} \\
\theta_{2} \\
\eta^{1} \\
\eta^{2} \\
\pi_{3} \\
\pi_{4}
\end{array}\right],
$$

with $a_{1}, a_{2}, c \neq 0$. Let $G \subset G L(7, \mathbb{R})$ be the group of such transformations, and let $\mathcal{P}$ be the $G$-structure on $\mathcal{M}^{\prime}$ of which the coframing of Lemma 4.2 is a section.

After absorbing as much torsion as possible and differentiating to uncover relations among the torsion, $\mathcal{P}$ has structure equations 
$\left[\begin{array}{l}d \theta \\ d \theta_{1} \\ d \theta_{2} \\ d \eta^{1} \\ d \eta^{2} \\ d \pi_{3} \\ d \pi_{4}\end{array}\right]=-\left[\begin{array}{ccccccc}\gamma & 0 & 0 & 0 & 0 & 0 & 0 \\ 0 & \gamma+\alpha_{1} & 0 & 0 & 0 & 0 & 0 \\ 0 & 0 & \gamma+\alpha_{2} & 0 & 0 & 0 & 0 \\ 0 & 0 & 0 & -\alpha_{1} & 0 & 0 & 0 \\ 0 & 0 & 0 & 0 & -\alpha_{2} & 0 & 0 \\ 0 & 0 & 0 & \beta_{1} & 0 & \gamma+2 \alpha_{1} & 0 \\ 0 & 0 & 0 & 0 & \beta_{2} & 0 & \gamma+2 \alpha_{2}\end{array}\right] \wedge\left[\begin{array}{c}\theta \\ \theta_{1} \\ \theta_{2} \\ \eta^{1} \\ \eta^{2} \\ \pi_{3} \\ \pi_{4}\end{array}\right]$

$$
-\left[\begin{array}{c}
\theta_{1} \wedge \eta^{1}+\theta_{2} \wedge \eta^{2} \\
\pi_{3} \wedge \eta^{1}+\left(A_{2} \theta_{2}+B_{2} \eta^{2}\right) \wedge \theta \\
\pi_{4} \wedge \eta^{2}+\left(A_{1} \theta_{1}+B_{1} \eta^{1}\right) \wedge \theta \\
0 \\
0 \\
2 C_{1} \theta_{1} \wedge \pi_{3} \\
2 C_{2} \theta_{2} \wedge \pi_{4}
\end{array}\right]
$$

Because of the dimensions of the derived flags of the characteristic systems (given in Lemma 4.2), $A_{1}, B_{1}$ are not both zero, and $A_{2}, B_{2}$ are not both zero. Furthermore, we can choose a local section $\sigma: \mathcal{M}^{\prime} \rightarrow \mathcal{P}$ satisfying the conditions that $\eta^{1}=d x$, $\eta^{2}=d y$, and the forms $\pi_{3}, \pi_{4}$ are integrable; i.e.,

$$
d \pi_{3} \equiv 0 \quad \bmod \pi_{3}, \quad d \pi_{4} \equiv 0 \quad \bmod \pi_{4}
$$

To see why, note that $\left\{\eta^{1}, \pi_{3}\right\}$ is a Frobenius system, and so it is spanned locally by two exact 1 -forms. Thus we can adjust $\pi_{3}$ by adding multiples of $\eta^{1}$ in order to make it a multiple of an exact form. Similarly, we can add multiples of $\eta^{2}$ to $\pi_{4}$ in order to make $\pi_{4}$ a multiple of an exact form. However, we cannot independently scale $\pi_{3}$ and $\pi_{4}$ to make both of them exact.

This choice of section is not unique; it is determined up to a transformation of the form

$$
\left[\begin{array}{c}
\tilde{\theta} \\
\tilde{\theta}_{1} \\
\tilde{\theta}_{2} \\
\tilde{\eta}^{1} \\
\tilde{\eta}^{2} \\
\tilde{\pi}_{3} \\
\tilde{\pi}_{4}
\end{array}\right]=\left[\begin{array}{lllllll}
c & 0 & 0 & 0 & 0 & 0 & 0 \\
0 & c & 0 & 0 & 0 & 0 & 0 \\
0 & 0 & c & 0 & 0 & 0 & 0 \\
0 & 0 & 0 & 1 & 0 & 0 & 0 \\
0 & 0 & 0 & 0 & 1 & 0 & 0 \\
0 & 0 & 0 & 0 & 0 & c & 0 \\
0 & 0 & 0 & 0 & 0 & 0 & c
\end{array}\right]^{-1}\left[\begin{array}{c}
\theta \\
\theta_{1} \\
\theta_{2} \\
\eta^{1} \\
\eta^{2} \\
\pi_{3} \\
\pi_{4}
\end{array}\right]
$$


with $c \neq 0$. However, we can make the choice of $\sigma$ unique (albeit slightly noncanonical) as follows: since $\pi_{3}, \pi_{4}$ are integrable 1 -forms, we must have

$$
\pi_{3}=e^{g} d \xi_{1}, \quad \pi_{4}=e^{h} d \xi_{2}
$$

for some functions $\xi_{1}, \xi_{2}, f, g$ on $\mathcal{M}^{\prime}$. Using the remaining scaling freedom, we can arrange that $h=-g$; the resulting coframing $\sigma: \mathcal{P} \rightarrow \mathcal{M}$ is uniquely determined.

When we pull back the structure equations via $\sigma$, the pseudoconnection forms $\alpha_{1}$, $\alpha_{2}, \beta_{1}, \beta_{2}, \gamma$ become semi-basic. By making use of the remaining ambiguity in these forms and the conditions imposed thus far on the coframing, we can assume that

$$
\begin{aligned}
\alpha_{1} & =\left(D_{1}+E_{1}\right) \eta^{1} \\
\alpha_{2} & =\left(D_{2}+E_{2}\right) \eta^{2} \\
\beta_{1} & =2 D_{1} \pi_{3} \\
\beta_{2} & =2 D_{2} \pi_{4} \\
\gamma & =-C_{1} \theta_{1}-C_{2} \theta_{2}-E_{1} \eta^{1}-E_{2} \eta^{2}+F_{1} \pi_{3}+F_{2} \pi_{4}
\end{aligned}
$$

for some functions $C_{i}, D_{i}, E_{i}, F_{i}$. Then the structure equations for this coframing become:

$$
\begin{aligned}
d \theta & =\theta \wedge\left(-C_{1} \theta_{1}-C_{2} \theta_{2}-E_{1} \eta^{1}-E_{2} \eta^{2}+F_{1} \pi_{3}+F_{2} \pi_{4}\right)-\theta_{1} \wedge \eta^{1}-\theta_{2} \wedge \eta^{2} \\
d \theta_{1} & =\theta_{1} \wedge\left(-C_{2} \theta_{2}-E_{2} \eta^{2}+F_{1} \pi_{3}+F_{2} \pi_{4}\right)+D_{1} \theta_{1} \wedge \eta^{1}-\pi_{3} \wedge \eta^{1}+\theta \wedge\left(A_{2} \theta_{2}+B_{2} \eta^{2}\right) \\
d \theta_{2} & =\theta_{2} \wedge\left(-C_{1} \theta_{1}-E_{1} \eta^{1}+F_{1} \pi_{3}+F_{2} \pi_{4}\right)+D_{2} \theta_{2} \wedge \eta^{2}-\pi_{4} \wedge \eta^{2}+\theta \wedge\left(A_{1} \theta_{1}+B_{1} \eta^{1}\right) \\
d \eta^{1} & =0 \\
d \eta^{2} & =0 \\
d \pi_{3} & =\pi_{3} \wedge\left(C_{1} \theta_{1}-C_{2} \theta_{2}+E_{1} \eta^{1}-E_{2} \eta^{2}+F_{2} \pi_{4}\right) \\
d \pi_{4} & =\pi_{4} \wedge\left(-C_{1} \theta_{1}+C_{2} \theta_{2}-E_{1} \eta^{1}+E_{2} \eta^{2}+F_{1} \pi_{3}\right) .
\end{aligned}
$$

(Note that these torsion functions are completely unrelated to those in $\S 2$ and $\S 3$.)

As in $\S 3$, we will need to compute relations among the derivatives of the torsion functions in order to show that $(\mathcal{M}, \mathcal{I})$ has a Bäcklund transformation to the wave equation. We begin by differentiating the structure equations (4.6). Using notation similar to that in $\S 3$, we denote derivatives as, e.g.,

$$
d A_{1}=A_{1,0} \theta+A_{1,1} \theta_{1}+A_{1,2} \theta_{2}+A_{1,3} \eta^{1}+A_{1,4} \eta^{2}+A_{1,5} \pi_{3}+A_{1,6} \pi_{4} .
$$

(Note that since this coframing is defined on a different manifold from that in $\S 3$, the indexing of the derivatives is different as well.)

Computing $d(d \theta)=d\left(d \theta_{1}\right)=d\left(d \theta_{2}\right)=d\left(d \pi_{3}\right)=d\left(d \pi_{4}\right)=0$ yields the following 
equations for the derivatives of the torsion functions:

$$
\begin{aligned}
& d A_{1}=A_{1,0} \theta+A_{1,1} \theta_{1}-3 A_{1} C_{2} \theta_{2}+A_{1,3} \eta^{1}-A_{1}\left(D_{2}+2 E_{2}\right) \eta^{2}+A_{1} F_{3} \pi_{3}+A_{1} F_{2} \pi_{4} \\
& d A_{2}=A_{2,0} \theta-3 A_{2} C_{1} \theta_{1}+A_{2,2} \theta_{2}-A_{2}\left(D_{1}+2 E_{1}\right) \eta^{1}+A_{2,4} \eta^{2}+A_{2} F_{3} \pi_{3}+A_{2} F_{2} \pi_{4} \\
& d B_{1}=B_{1,0} \theta+\left(A_{1,3}-A_{1} D_{1}\right) \theta_{1}-2 B_{1} C_{2} \theta_{2}+B_{1,3} \eta^{1}-B_{1}\left(D_{2}+E_{2}\right) \eta^{2}+A_{1} \pi_{3} \\
& d B_{2}=B_{2,0} \theta-2 B_{2} C_{1} \theta_{1}+\left(A_{2,4}-A_{2} D_{2}\right) \theta_{2}-B_{2}\left(D_{1}+E_{1}\right) \eta^{1}+B_{2,4} \eta^{2}+A_{2} \pi_{4} \\
& d C_{1}=A_{1} C_{2} \theta+C_{1,1} \theta_{1}-C_{1} C_{2} \theta_{2}+C_{1,3} \eta^{1}-\left(\frac{1}{2} A_{1}+C_{1} E_{2}\right) \eta^{2}+C_{1,5} \pi_{3}+C_{1} F_{2} \pi_{4} \\
& d C_{2}=A_{2} C_{1} \theta-C_{1} C_{2} \theta_{1}+C_{2,2} \theta_{2}-\left(\frac{1}{2} A_{2}+C_{2} E_{1}\right) \eta^{1}+C_{2,4} \eta^{2}+C_{2} F_{1} \pi_{3}+C_{2,6} \pi_{4} \\
& d D_{1}=B_{1} C_{2} \theta+D_{1,1} \theta_{1}+\frac{3}{2} A_{2} \theta_{2}+D_{1,3} \eta^{1}+\frac{1}{2}\left(3 B_{2}-B_{1}\right) \eta^{2}+\left(2 C_{1}-E_{1,5}\right) \pi_{3} \\
& d D_{2}=B_{2} C_{1} \theta+\frac{3}{2} A_{1} \theta_{1}+D_{2,2} \theta_{2}+\frac{1}{2}\left(3 B_{1}-B_{2}\right) \eta^{1}+D_{2,4} \eta^{2}+\left(2 C_{2}-E_{2,6}\right) \pi_{4} \\
& d E_{1}=B_{1} C_{2} \theta+\left(C_{1,3}-C_{1} D_{1}\right) \theta_{1}+\frac{1}{2} A_{2} \theta_{2}+E_{1,3} \eta^{1}+\frac{1}{2}\left(B_{2}-B_{1}\right) \eta^{2}+E_{1,5} \pi_{3} \\
& d E_{2}=B_{2} C_{1} \theta+\frac{1}{2} A_{1} \theta_{1}+\left(C_{2,4}-C_{2} D_{2}\right) \theta_{2}+\frac{1}{2}\left(B_{1}-B_{2}\right) \eta^{1}+E_{2,4} \eta^{2}+E_{2,6} \pi_{4} \\
& d F_{1}=\left(2 C_{1} F_{1}-C_{1,5}\right) \theta_{1}-C_{2} F_{1} \theta_{2}+\left(C_{1}+E_{1} F_{1}-E_{1,5}\right) \eta^{1}-E_{2} F_{1} \eta^{2}+F_{1,5} \pi_{3}+F_{1,6} \pi_{4} \\
& d F_{2}=-C_{1} F_{2} \theta_{1}+\left(2 C_{2} F_{2}-C_{2,6}\right) \theta_{2}-E_{1} F_{2} \eta^{1}+\left(C_{2}+E_{2} F_{2}-E_{2,6}\right) \eta^{2}+F_{1,6} \pi_{3}+F_{2,6} \pi_{4} .
\end{aligned}
$$

Because $A_{1}$ appears as a derivative of $B_{1}$, and $A_{1}, B_{1}$ cannot vanish simultaneously, $B_{1}$ cannot vanish on any open set in $\mathcal{M}^{\prime}$. In fact, $B_{1}$ cannot vanish identically on any fiber of the projection $\mathcal{M}^{\prime} \rightarrow \mathcal{M}$, and the same is true of $B_{2}$. Henceforth we restrict to the dense open set in $\mathcal{M}^{\prime}$ where $B_{1}, B_{2}$ are both nonzero, and note that this set surjects onto $\mathcal{M}$.

We may obtain further relations among the derivatives of the torsion functions by differentiating equations (4.7). Computing $d\left(d A_{1}\right) \equiv d\left(d B_{1}\right) \equiv 0$ modulo $\theta, \theta_{1}, \eta^{1}, \pi_{4}$ yields

$$
A_{1,0}=A_{1}\left(C_{2,4}-D_{2,2}-C_{2} D_{2}\right), \quad B_{1,0}=B_{1}\left(C_{2,4}-D_{2,2}-C_{2} D_{2}\right) .
$$

Then computing $d\left(d B_{1}\right) \equiv 0$ modulo $\theta, \theta_{1}, \eta^{1}$ yields $C_{2,6}=C_{2} F_{2}$. Similar considerations of $d\left(d A_{2}\right)$ and $d\left(d B_{2}\right)$ show that

$A_{2,0}=A_{2}\left(C_{1,3}-D_{1,1}-C_{1} D_{1}\right), \quad B_{2,0}=B_{2}\left(C_{1,3}-D_{1,1}-C_{1} D_{1}\right), \quad C_{1,5}=C_{1} F_{1}$.

It will now be convenient to derive several equations and solve them simultaneously. First, $d\left(d C_{1}\right) \equiv 0$ modulo $\theta_{1}, \eta^{1}$ implies that

$$
\begin{gathered}
A_{1}\left(C_{2,2}-C_{2}^{2}\right)=A_{2}\left(C_{1,1}-C_{1}^{2}\right) \\
A_{1}\left(3 C_{2,4}-D_{2,2}-3 C_{2} D_{2}\right)=2 B_{2}\left(C_{1,1}-C_{1}^{2}\right) .
\end{gathered}
$$

Additionally, $d\left(d C_{2}\right) \equiv 0$ modulo $\theta_{2}, \eta^{2}$ implies that

$$
A_{2}\left(3 C_{1,3}-D_{1,1}-3 C_{1} D_{1}\right)=2 B_{1}\left(C_{2,2}-C_{2}^{2}\right) .
$$

Finally, $d\left(d D_{1}\right) \equiv 0$ modulo $\theta_{1}, \eta^{1}, \pi_{3}$ implies that

$$
B_{1}\left(3 C_{2,4}-D_{2,2}-3 C_{2} D_{2}\right)=B_{2}\left(3 C_{1,3}-D_{1,1}-3 C_{1} D_{1}\right) .
$$

The general solution to equations (4.8)-(4.11) is most easily expressed in terms of a new torsion function $H$, such that

$$
\begin{array}{ll}
C_{1,1}=C_{1}^{2}-A_{1} H, & D_{1,1}=3\left(C_{1,3}-C_{1} D_{1}\right)+2 B_{1} H, \\
C_{2,2}=C_{2}^{2}-A_{2} H, & D_{2,2}=3\left(C_{2,4}-C_{2} D_{2}\right)+2 B_{2} H .
\end{array}
$$


Next, we need information about the derivatives of $C_{1,3}$ and $C_{2,4}$. Computing $d\left(d C_{1}\right) \equiv 0$ modulo $\theta_{1}$ yields

$$
\begin{gathered}
d C_{1,3}=\left(C_{2} A_{1,3}-\frac{1}{2} A_{1} A_{2}+B_{1} C_{1} C_{2}\right) \theta+C_{1,31} \theta_{1}+\left(\frac{1}{2} A_{2} C_{1}-C_{2} C_{1,3}\right) \theta_{2} \\
+C_{1,33} \eta^{1}-\frac{1}{2}\left(A_{1,3}+2 E_{2} C_{1,3}+C_{1}\left(B_{1}-B_{2}\right)\right) \eta^{2} \\
+\left(F_{1} C_{1,3}-C_{1} E_{1,5}+2 C_{1}^{2}-A_{1} H\right) \pi_{3}+F_{2} C_{1,3} \pi_{4}
\end{gathered}
$$

and computing $d\left(d C_{2}\right) \equiv 0$ modulo $\theta_{2}$ yields

$$
\begin{gathered}
d C_{2,4}=\left(C_{1} A_{2,4}-\frac{1}{2} A_{1} A_{2}+B_{2} C_{1} C_{2}\right) \theta+\left(\frac{1}{2} A_{1} C_{2}-C_{1} C_{2,4}\right) \theta_{1}+C_{2,42} \theta_{2} \\
-\frac{1}{2}\left(A_{2,4}+2 E_{1} C_{2,4}+C_{2}\left(B_{2}-B_{1}\right)\right) \eta^{1}+C_{2,44} \eta^{2} \\
+F_{1} C_{2,4} \pi_{3}+\left(F_{2} C_{2,4}-C_{2} E_{2,4}+2 C_{2}^{2}-A_{2} H\right) \pi_{4} .
\end{gathered}
$$

Now, computing $d\left(d C_{1}\right)=d\left(d C_{2}\right)=0, d\left(d D_{1}\right) \equiv 0$ modulo $\eta^{1}, \pi_{3}$, and $d\left(d D_{2}\right) \equiv$ 0 modulo $\eta^{2}, \pi_{4}$ yields four different expressions for $d H$. Taking linear combinations of these expressions shows that

$$
\begin{aligned}
& A_{1}\left(A_{1,3}-A_{1} D_{1}\right)=B_{1}\left(A_{1,1}-A_{1} C_{1}\right) \\
& A_{2}\left(A_{2,4}-A_{2} D_{2}\right)=B_{2}\left(A_{2,2}-A_{2} C_{2}\right) .
\end{aligned}
$$

Equations (4.12) and (4.13) may be solved by introducing new torsion functions $J_{1}, J_{2}$, such that

$$
\begin{array}{ll}
A_{1,1}=A_{1} C_{1}+A_{1} J_{1} & A_{2,2}=A_{2} C_{2}+A_{2} J_{2} \\
A_{1,3}=A_{1} D_{1}+B_{1} J_{1} & A_{2,4}=A_{2} D_{2}+B_{2} J_{2} .
\end{array}
$$

Then the various expressions for $d H$ may be combined to show that

$$
\begin{aligned}
& C_{1,31}=2 C_{1} C_{1,3}-C_{1}^{2} D_{1}+A_{1} C_{2}-\frac{1}{2} A_{1} J_{2}-\left(A_{1} D_{1}+B_{1} J_{1}\right) H \\
& C_{2,42}=2 C_{2} C_{2,4}-C_{2}^{2} D_{2}+A_{2} C_{1}-\frac{1}{2} A_{2} J_{1}-\left(A_{2} D_{2}+B_{2} J_{2}\right) H,
\end{aligned}
$$

and

$$
\begin{aligned}
d H= & \left(H\left(C_{1,3}-C_{1} D_{1}+B_{1} H+C_{2,4}-C_{2} D_{2}+B_{2} H\right)-\frac{1}{2} C_{1} J_{2}+C_{2} J_{1}\right) \theta \\
& +C_{1} H \theta_{1}+C_{2} H \theta_{2}+\left(D_{1} H+\frac{1}{2} J_{2}\right) \eta^{1}+\left(D_{2} H+\frac{1}{2} J_{1}\right) \eta^{2}+F_{1} H \pi_{3}+F_{2} H \pi_{4} .
\end{aligned}
$$

They also imply the relation

$$
H\left(C_{1,3}-C_{1} D_{1}+B_{1} H\right)-\frac{1}{2} C_{1} J_{2}=H\left(C_{2,4}-C_{2} D_{2}+B_{2} H\right)-\frac{1}{2} C_{2} J_{1} .
$$

The equations for $d A_{1}, d A_{2}$ now take the form:

$$
\begin{gathered}
d A_{1}=-2 A_{1}\left(C_{2,4}-C_{2} D_{2}+B_{2} H\right) \theta+A_{1}\left(C_{1}+J_{1}\right) \theta_{1}-3 A_{1} C_{2} \theta_{2} \\
+\left(A_{1} D_{1}+B_{1} J_{1}\right) \eta^{1}-A_{1}\left(D_{2}+2 E_{2}\right) \eta^{2}+A_{1} F_{3} \pi_{3}+A_{1} F_{2} \pi_{4} \\
d A_{2}=-2 A_{2}\left(C_{1,3}-C_{1} D_{1}+B_{1} H\right) \theta-3 A_{2} C_{1} \theta_{1}+A_{2}\left(C_{2}+J_{2}\right) \theta_{2} \\
-A_{2}\left(D_{1}+2 E_{1}\right) \eta^{1}+\left(A_{2} D_{2}+B_{2} J_{2}\right) \eta^{2}+A_{2} F_{3} \pi_{3}+A_{2} F_{2} \pi_{4} .
\end{gathered}
$$


Now, computing $d\left(d B_{1}\right) \equiv 0$ modulo $\theta_{1}, \eta^{1}$ yields

$C_{2,44}=\left(D_{2}-E_{2}\right) C_{2,4}+C_{2} D_{2,4}-H B_{2,4}-\left(D_{2}+E_{2}\right) B_{2} H+C_{2} D_{2} E_{2}+B_{2}\left(C_{1}-J_{1}\right)$.

Similarly, computing $d\left(d B_{2}\right) \equiv 0$ modulo $\theta_{2}, \eta^{2}$ yields

$C_{1,33}=\left(D_{1}-E_{1}\right) C_{1,3}+C_{1} D_{1,3}-H B_{1,3}-\left(D_{1}+E_{1}\right) B_{1} H+C_{1} D_{1} E_{1}+B_{1}\left(C_{2}-J_{2}\right)$.

Next, computing $d\left(d A_{1}\right)=0$ shows that

$$
\begin{aligned}
d J_{1}=4 A_{1} C_{2} \theta+\left(C_{1} J_{1}+A_{1} K_{1}\right) \theta_{1}-C_{2} J_{1} \theta_{2} & \\
+ & \left(2 C_{1,3}-2 C_{1} D_{1}+2 B_{1} H+2 C_{2,4}-2 C_{2} D_{2}+2 B_{2} H+D_{1} J_{1}+B_{1} K_{1}\right) \eta^{1} \\
& \quad-\left(2 A_{1}+E_{2} J_{1}\right) \eta^{2}+F_{1} J_{1} \pi_{3}+F_{2} J_{1} \pi_{4}
\end{aligned}
$$

for some function $K_{1}$. Similarly, computing $d\left(d A_{2}\right)=0$ shows that

$$
\begin{aligned}
d J_{2}= & 4 A_{2} C_{1} \theta-C_{1} J_{2} \theta_{1}+\left(C_{2} J_{2}+A_{2} K_{2}\right) \theta_{2}-\left(2 A_{2}+E_{1} J_{2}\right) \eta^{1} \\
& +\left(2 C_{1,3}-2 C_{1} D_{1}+2 B_{1} H+2 C_{2,4}-2 C_{2} D_{2}+2 B_{2} H+D_{2} J_{2}+B_{2} K_{2}\right) \eta^{2} \\
& \quad+F_{1} J_{2} \pi_{3}+F_{2} J_{2} \pi_{4}
\end{aligned}
$$

for some function $K_{2}$. But now computing $d(d H) \equiv 0$ modulo $\theta, \theta_{1}, \theta_{2}$ yields

$$
B_{1}\left(K_{1}+4 H\right)=B_{2}\left(K_{2}+4 H\right) .
$$

It follows that

$$
\begin{aligned}
& K_{1}=-4 H+B_{2} M \\
& K_{2}=-4 H+B_{1} M
\end{aligned}
$$

for some function $M$. Computing $d(d H) \equiv 0$ modulo $\theta_{2}, \eta^{1}$ and $d(d H) \equiv 0$ modulo $\theta_{1}, \eta^{2}$ shows that

$$
A_{1} M=A_{2} M=0
$$

Claim. $M=0$.

Proof. Suppose not. Then by (4.17), $A_{1}=A_{2}=0$. Therefore, equations (4.15) and (4.16) reduce to

$$
\begin{gathered}
0=d A_{1}=B_{1} J_{1} \eta^{1} \\
0=d A_{2}=B_{2} J_{2} \eta^{2} .
\end{gathered}
$$

Since $B_{1}, B_{2}$ are nonzero, it follows that $J_{1}=J_{2}=0$. Then

$$
\begin{aligned}
& 0=d J_{1}=\left(2 C_{1,3}-2 C_{1} D_{1}-2 B_{1} H+2 C_{2,4}-2 C_{2} D_{2}+2 B_{2} H+B_{1} B_{2} M\right) \eta^{1} \\
& 0=d J_{2}=\left(2 C_{1,3}-2 C_{1} D_{1}+2 B_{1} H+2 C_{2,4}-2 C_{2} D_{2}-2 B_{2} H+B_{1} B_{2} M\right) \eta^{2} .
\end{aligned}
$$

Subtracting the two coefficients above yields

$$
4\left(B_{2}-B_{1}\right) H=0
$$

so either $B_{1}=B_{2}$ or $H=0$. 
First suppose that $B_{1}=B_{2}$. Then

$$
0=d\left(B_{2}-B_{1}\right) \equiv-2 B_{1}\left(C_{1} \theta_{1}-C_{2} \theta_{2}\right) \bmod \theta, \eta^{1}, \eta^{2},
$$

so $C_{1}=C_{2}=0$. It follows that $C_{1,3}=C_{2,4}=0$ as well. But now

$$
0=d J_{1}=B_{1}^{2} M
$$

so $M=0$, as desired.

Now suppose that $H=0$. Computing $d\left(d C_{1,3}\right)-C_{1} d\left(d D_{1}\right) \equiv 0$ modulo $\theta$ yields

$$
B_{1}^{2} B_{2} M=0
$$

so $M=0$ in this case as well.

Finally, computing $d(d H)=0$, keeping the relation (4.14) in mind, yields two additional relations:

$$
\begin{aligned}
& 2 C_{2}\left(2 C_{1,3}-2 C_{1} D_{1}+2 B_{1} H\right) \\
& +\left(2 C_{2}-J_{2}\right)\left(2 C_{2,4}-2 C_{2} D_{2}+2 B_{2} H\right)+A_{2}\left(4 C_{1}-J_{1}\right)=0 \\
& \left(2 C_{1}-J_{1}\right)\left(2 C_{1,3}-2 C_{1} D_{1}+2 B_{1} H\right) \\
& \quad+2 C_{1}\left(2 C_{2,4}-2 C_{2} D_{2}+2 B_{2} H\right)+A_{1}\left(4 C_{2}-J_{2}\right)=0 .
\end{aligned}
$$

We now have all the relations that will be needed for the involutivity calculation below.

Now suppose that $\overline{\mathcal{M}}=\mathbb{R}^{5}$ carries a Monge-Ampère system $\overline{\mathcal{I}}$ representing the wave equation $Z_{X Y}=0$, generated algebraically by the contact form

$$
\bar{\theta}=d Z-P d X-Q d Y
$$

and the 2-forms $d P \wedge d X$ and $d Q \wedge d Y$. If there were a Bäcklund transformation $\mathcal{B} \subset \mathcal{M} \times \overline{\mathcal{M}}$, then $Z$ would be a local coordinate on the fibers of $\mathcal{B} \rightarrow \mathcal{M}$ and the functions $X, Y, P, Q$ on $\mathcal{B}$ would satisfy the Bäcklund condition

$$
\{d P \wedge d X, d Q \wedge d Y\} \equiv\left\{\pi_{1} \wedge \eta^{1}, \pi_{2} \wedge \eta^{2}\right\} \bmod \theta, \bar{\theta}
$$

(see the definition at the beginning of $\S 2$ ).

Accordingly, we let $\mathcal{B}=\mathcal{M} \times \mathbb{R}$, with coordinate $Z$ on the second factor. We will show that, on an open neighborhood of any point of $\mathcal{B}$, there exist functions $X, Y, P, Q$ such that the ideal $\mathcal{J}=\mathcal{I} \cup\{\bar{\theta}\}$ on $\mathcal{B}$ (where $\bar{\theta}$ is defined as in (4.20)) gives a Bäcklund transformation between $(\mathcal{M}, \mathcal{I})$ and $(\overline{\mathcal{M}}, \overline{\mathcal{I}})$. We will do this by setting up an EDS whose integral manifolds correspond to functions satisfying these conditions; once we know that this EDS is involutive, an application of the Cartan-Kähler Theorem will prove the existence of the desired Bäcklund transformations.

Let $\mathcal{B}^{\prime}=\mathcal{M}^{\prime} \times \mathbb{R}$, again with $Z$ as the coordinate on the second factor; we extend the projection $\mathcal{M}^{\prime} \rightarrow \mathcal{M}$ to a projection $\mathcal{B}^{\prime} \rightarrow \mathcal{B}$ by the identity on the second factor. It will be convenient to set up our EDS in terms of the coframing $\left(\theta, \bar{\theta}, \theta_{1}, \theta_{2}, \eta^{1}, \eta^{2}, \pi_{3}, \pi_{4}\right)$ on $\mathcal{B}^{\prime}$. Thus, we will regard $X, Y, P, Q$ as functions on $\mathcal{B}^{\prime}$, but require that

$$
d X, d Y, d P, d Q \in\left\{\theta, \bar{\theta}, \theta_{1}, \theta_{2}, \eta^{1}, \eta^{2}\right\}
$$


so that they are in fact well-defined on $\mathcal{B}$. In order to satisfy the Bäcklund condition (4.21), we will furthermore require that

$$
\{d X, d P\} \subset\left\{\theta, \bar{\theta}, \theta_{1}, \eta^{1}\right\}, \quad\{d Y, d Q\} \subset\left\{\theta, \bar{\theta}, \theta_{2}, \eta^{2}\right\} .
$$

From this, and the structure equations (4.6), it follows that $\{d X, d P\}$ (resp., $\{d Y, d Q\}$ ) is the largest integrable subsystem of $\left\{\theta, \bar{\theta}, \theta_{1}, \eta^{1}\right\}$ (resp., $\left\{\theta, \bar{\theta}, \theta_{2}, \eta^{2}\right\}$ ). Therefore,

$$
\eta^{1}=d x \in\{d X, d P\}, \quad \eta^{2}=d y \in\{d Y, d Q\},
$$

and by a contact transformation on $\overline{\mathcal{M}}$, we may assume that $X=x, Y=y$. Thus, we will set

$$
\bar{\theta}=d Z-P d x-Q d y
$$

and condition (4.22) becomes

$$
d P \in\left\{\theta, \bar{\theta}, \theta_{1}, \eta^{1}\right\}, \quad d Q \in\left\{\theta, \bar{\theta}, \theta_{2}, \eta^{2}\right\} .
$$

Suppose that

$$
\begin{aligned}
& d P=P_{0} \theta+P_{\overline{0}} \bar{\theta}+P_{1} \theta_{1}+P_{3} \eta^{1} \\
& d Q=Q_{0} \theta+Q_{\overline{0}} \bar{\theta}+Q_{2} \theta_{2}+Q_{4} \eta^{2} .
\end{aligned}
$$

Observe that normality of the Bäcklund transformation requires that $P_{1}, Q_{2} \neq 0$ and $P_{1} \neq Q_{2}$.

REMARK. Equations (4.23)-(4.24) give an overdetermined system of first-order partial differential equations for functions $P$ and $Q$. The process of generating compatibility conditions for such systems can be carried out systematically by computing the exterior derivatives of the 1-form equations, and using the fact that the repeated exterior derivative of a function is zero. Moreover, applying Cartan's Test for involutivity (see [8], Chapter 7) to the resulting EDS will tell us when we can stop differentiating: if the system is involutive then no further compatibility conditions arise through differentiation, and solutions exist that may be constructed by applying the Cartan-Kähler Theorem.

Differentiating (4.23) modulo $\theta, \bar{\theta}, \theta_{1}, \eta^{1}$ yields

$$
\left(P_{0}+P_{\overline{0}} Q_{2}\right) \theta_{2} \wedge \eta^{2}=0
$$

and differentiating (4.24) modulo $\theta, \bar{\theta}, \theta_{2}, \eta^{2}$ yields

$$
\left(Q_{0}+Q_{\overline{0}} P_{1}\right) \theta_{1} \wedge \eta^{1}=0 .
$$

Therefore, because the 1 -forms $\theta, \bar{\theta}, \theta_{1}, \theta_{2}, \eta^{1}, \eta^{2}$ are linearly independent on $\mathcal{B}$, we have $P_{0}=-P_{\overline{0}} Q_{2}, Q_{0}=-Q_{\overline{0}} P_{1}$, and we may write

$$
\begin{aligned}
& d P=P_{\overline{0}}\left(\bar{\theta}-Q_{2} \theta\right)+P_{1} \theta_{1}+P_{3} \eta^{1} \\
& d Q=Q_{\overline{0}}\left(\bar{\theta}-P_{1} \theta\right)+Q_{2} \theta_{2}+Q_{4} \eta^{2} .
\end{aligned}
$$

Note that neither $P_{\overline{0}}$ nor $Q_{\overline{0}}$ can vanish identically: for, if $P_{\overline{0}}=0$, then differentiating (4.25) shows that $P_{1}=0$ as well, which contradicts the hypothesis of normality. (A similar argument applies to $Q_{\overline{0}}$.) 
Differentiating equations (4.25) modulo various combinations of 1-forms leads to the following expressions for the exterior derivatives of $P_{\overline{0}}, P_{1}, P_{3}, Q_{\overline{0}}, Q_{2}, Q_{4}$ :

$$
\begin{aligned}
d P_{\overline{0}}= & -P_{\overline{0}} Q_{\overline{0} 2} \theta+P_{\overline{00}}\left(\bar{\theta}-Q_{2} \theta\right)+P_{\overline{0} 1} \theta_{1}+P_{\overline{0} 3} \eta^{1} \\
d P_{1}= & P_{\overline{0} 1}\left(\bar{\theta}-Q_{2} \theta\right)-\frac{\left(A_{1} Q_{2}+C_{1} Q_{\overline{0}} P_{1}\right)}{Q_{\overline{0}}} \theta_{1}-C_{2} P_{1} \theta_{2} \\
& \quad-\frac{\left(B_{1} Q_{2}+Q_{\overline{0}}\left(E_{1} P_{1}+P_{\overline{0}}\left(Q_{2}-P_{1}\right)\right)\right)}{Q_{\overline{0}}} \eta^{1}-E_{2} P_{1} \eta^{2}+F_{1} P_{1} \pi_{3}+F_{2} P_{1} \pi_{4} \\
d P_{3}= & \left(P_{\overline{0} 3}+P_{\overline{0}}^{2}\right)\left(\bar{\theta}-Q_{2} \theta\right)-\frac{\left(B_{1} Q_{2}+Q_{\overline{0}}\left(\left(D_{1}+E_{1}\right) P_{1}+2 P_{\overline{0}}\left(Q_{2}-P_{1}\right)\right)\right)}{Q_{\overline{0}}} \theta_{1} \\
& \quad+P_{33} \eta^{1}+P_{1} \pi_{3} \\
d Q_{\overline{0}}= & -Q_{\overline{0}} P_{\overline{0} 1} \theta+Q_{\overline{00}}\left(\bar{\theta}-P_{1} \theta\right)+Q_{\overline{0} 2} \theta_{2}+Q_{\overline{0} 4} \eta^{2} \\
d Q_{2}= & Q_{\overline{0} 2}\left(\bar{\theta}-P_{1} \theta\right)-C_{1} Q_{2} \theta_{1}-\frac{\left(A_{2} P_{1}+C_{2} P_{\overline{0}} Q_{2}\right)}{P_{\overline{0}}} \theta_{2} \\
& \quad-E_{1} Q_{2} \eta^{2}-\frac{\left(B_{2} P_{1}+P_{\overline{0}}\left(E_{2} Q_{2}+Q_{\overline{0}}\left(P_{1}-Q_{2}\right)\right)\right)}{P_{\overline{0}}} \eta^{2}+F_{1} Q_{2} \pi_{3}+F_{2} Q_{2} \pi_{4} \\
d Q_{4}= & \left(Q_{\overline{0} 4}+Q_{\overline{0}}^{2}\right)\left(\bar{\theta}-P_{1} \theta\right)-\frac{\left(B_{2} P_{1}+P_{\overline{0}}\left(\left(D_{2}+E_{2}\right) Q_{2}+2 Q_{\overline{0}}\left(P_{1}-Q_{2}\right)\right)\right)}{P_{\overline{0}}} \theta_{2} \\
& +Q_{44} \eta^{2}+Q_{2} \pi_{4} .
\end{aligned}
$$

Now computing $d\left(d P_{1}\right) \equiv 0$ modulo $\bar{\theta}-Q_{2} \theta$ and $d\left(d Q_{2}\right) \equiv 0$ modulo $\bar{\theta}-P_{1} \theta$ yields

$$
\begin{aligned}
P_{\overline{00}}= & \frac{1}{Q_{\overline{0}} P_{1}^{2} Q_{2}\left(Q_{2}-P_{1}\right)}\left(2 C_{1}\left(P_{1}-2 Q_{2}\right) P_{1} Q_{2} P_{\overline{0}}^{2} Q_{\overline{0}}+2 C_{2} P_{1}^{2} Q_{2} P_{\overline{0}} Q_{\overline{0}}^{2}\right. \\
& \left.+2\left(C_{1,3}-C_{1} D_{1}+B_{1} H\right) P_{1}^{2} Q_{2} P_{\overline{0}} Q_{\overline{0}}+A_{1}\left(P_{1}-Q_{2}\right) Q_{2}^{2} P_{\overline{0}}^{2}+A_{2} P_{1}^{3} Q_{\overline{0}}^{2}\right) \\
Q_{\overline{00}}= & \frac{1}{P_{\overline{0}} P_{1} Q_{2}^{2}\left(P_{1}-Q_{2}\right)}\left(2 C_{1} P_{1} Q_{2}^{2} P_{\overline{0}}^{2} Q_{\overline{0}}+2 C_{2}\left(Q_{2}-2 P_{1}\right) P_{1} Q_{2} P_{\overline{0}} Q_{\overline{0}}^{2}\right. \\
& \left.+2\left(C_{2,4}-C_{2} D_{2}+B_{2} H\right) P_{1} Q_{2}^{2} P_{\overline{0}} Q_{\overline{0}}+A_{1} Q_{2}^{3} P_{\overline{0}}^{2}+A_{2}\left(Q_{2}-P_{1}\right) P_{1}^{2} Q_{\overline{0}}^{2}\right) \\
P_{\overline{0} 1}=- & \frac{P_{\overline{0}}\left(A_{1} Q_{2}+2 C_{1} Q_{\overline{0}} P_{1}\right)}{Q_{\overline{0}} P_{1}} \\
Q_{\overline{0} 2}=- & \frac{Q_{\overline{0}}\left(A_{2} P_{1}+2 C_{2} P_{\overline{0}} Q_{2}\right)}{P_{\overline{0}} Q_{2}} \\
P_{\overline{0} 3}=- & \frac{P_{\overline{0}}\left(B_{1} Q_{2}+\left(D_{1}+E_{1}\right) P_{1} Q_{\overline{0}}+\left(2 Q_{2}-P_{1}\right) P_{\overline{0}} Q_{\overline{0}}\right)}{P_{1} Q_{\overline{0}}} \\
Q_{\overline{0} 4}=- & \frac{Q_{\overline{0}}\left(B_{2} P_{1}+\left(D_{2}+E_{2}\right) Q_{2} P_{\overline{0}}+\left(2 P_{1}-Q_{2}\right) P_{\overline{0}} Q_{\overline{0}}\right)}{Q_{2} P_{\overline{0}}} .
\end{aligned}
$$

This leaves only $P_{33}$ and $Q_{44}$ as undetermined second derivatives of $P$ and $Q$.

We are now ready to set up our exterior differential system. Let $\widehat{\mathcal{B}}=\mathcal{B} \times \mathbb{R}^{10}$, with coordinates $P, Q, P_{\overline{0}}, P_{1}, P_{3}, Q_{\overline{0}}, Q_{2}, Q_{4}, P_{33}, Q_{44}$ on the $\mathbb{R}^{10}$ factor. Let $\mathcal{W}$ be the rank 8 Pfaffian EDS on $\widehat{\mathcal{B}}$ generated by the 1 -forms

$$
\begin{aligned}
& \Theta_{1}=d P-P_{\overline{0}}\left(\bar{\theta}-Q_{2} \theta\right)-P_{1} \theta_{1}-P_{3} \eta^{1}, \\
& \Theta_{2}=d Q-Q_{\overline{0}}\left(\bar{\theta}-P_{1} \theta\right)-Q_{2} \theta_{2}-Q_{4} \eta^{2},
\end{aligned}
$$


and similar forms $\Theta_{3}, \ldots, \Theta_{8}$ prescribing conditions (4.26) for $d P_{\overline{0}}, d P_{1}, d P_{3}, d Q_{\overline{0}}$, $d Q_{2}$, and $d Q_{4}$, substituting the values (4.27) for $P_{\overline{00}}, P_{\overline{0} 1}, P_{\overline{0} 3}, Q_{\overline{00}}, Q_{\overline{0} 2}, Q_{\overline{0} 4}$. Integral manifolds of $\mathcal{W}$ satisfying the independence condition $\theta \wedge \bar{\theta} \wedge \theta_{1} \wedge \theta_{2} \wedge \eta^{1} \wedge \eta^{2} \wedge \pi_{3} \wedge \pi_{4} \neq 0$ are in one-to-one correspondence with the desired functions $P, Q$ defining a Bäcklund transformation.

The structure equations for this EDS have the form:

$$
\left[\begin{array}{c}
d \Theta_{1} \\
d \Theta_{2} \\
d \Theta_{3} \\
d \Theta_{4} \\
d \Theta_{5} \\
d \Theta_{6} \\
d \Theta_{7} \\
d \Theta_{8}
\end{array}\right] \equiv-\left[\begin{array}{llllllll}
0 & 0 & 0 & 0 & 0 & 0 & 0 & 0 \\
0 & 0 & 0 & 0 & 0 & 0 & 0 & 0 \\
0 & 0 & 0 & 0 & 0 & 0 & 0 & 0 \\
0 & 0 & 0 & 0 & 0 & 0 & 0 & 0 \\
0 & 0 & 0 & 0 & \Pi_{1} & 0 & 0 & 0 \\
0 & 0 & 0 & 0 & 0 & 0 & 0 & 0 \\
0 & 0 & 0 & 0 & 0 & 0 & 0 & 0 \\
0 & 0 & 0 & 0 & 0 & \Pi_{2} & 0 & 0
\end{array}\right] \wedge\left[\begin{array}{c}
\theta \\
\bar{\theta} \\
\theta_{1} \\
\theta_{2} \\
\eta^{1} \\
\eta^{2} \\
\pi_{3} \\
\pi_{4}
\end{array}\right]+\left[\begin{array}{c}
\Psi_{1} \\
\Psi_{2} \\
\Psi_{3} \\
\Psi_{4} \\
\Psi_{5} \\
\Psi_{6} \\
\Psi_{7} \\
\Psi_{8}
\end{array}\right] \quad \bmod \Theta_{1}, \ldots, \Theta_{8}
$$

where

$$
\left.\begin{array}{l}
\Pi_{1} \equiv d P_{33} \\
\Pi_{2} \equiv d Q_{44}
\end{array}\right\} \quad \bmod \theta, \bar{\theta}, \theta_{1}, \theta_{2}, \eta^{1}, \eta^{2}, \pi_{3}, \pi_{4},
$$

and $\Psi_{1}, \ldots, \Psi_{8}$ are 2 -forms which are quadratic in the forms $\theta, \bar{\theta}, \theta_{1}, \theta_{2}, \eta^{1}, \eta^{2}, \pi_{3}, \pi_{4}$, with coefficients which are polynomial functions of the two quantities

$$
\begin{gathered}
\left(2 H P_{1}\left(Q_{2}-P_{1}\right) Q_{\overline{0}}^{2}+Q_{2}\left(2 C_{1} Q_{2}+J_{1}\left(P_{1}-Q_{2}\right)\right) Q_{\overline{0}}+A_{1} Q_{2}^{2}\right) P_{\overline{0}} \\
-2 C_{2} P_{1}^{2} Q_{\overline{0}}^{2}+2\left(C_{2,4}-C_{2} D_{2}+B_{2} H\right) Q_{\overline{0}} P_{1} Q_{2}, \\
\left(2 H Q_{2}\left(P_{1}-Q_{2}\right) P_{\overline{0}}^{2}+P_{1}\left(2 C_{2} P_{1}+J_{2}\left(Q_{2}-P_{1}\right)\right) P_{\overline{0}}+A_{2} P_{1}^{2}\right) Q_{\overline{0}} \\
-2 C_{1} Q_{2}^{2} P_{\overline{0}}^{2}+2\left(C_{1,3}-C_{1} D_{1}+B_{1} H\right) P_{\overline{0}} P_{1} Q_{2} .
\end{gathered}
$$

In order to find integral manifolds, we must restrict $\mathcal{W}$ to the locus $Z \subset \widehat{\mathcal{B}}$ defined by the simultaneous vanishing of (4.29) and (4.30). (These relations could also be found more directly, by computing $d\left(d P_{1}\right)=d\left(d Q_{2}\right)=0$.) While relations of this sort could easily lead to hopeless incompatibility for the PDE system given by (4.25) and (4.26), it turns out that differentiating these quantities yields no new relations.

A case-by-case analysis, based on the vanishing or non-vanishing of various torsion coefficients of $(\mathcal{M}, \mathcal{I})$, shows that the functions $P_{\overline{0}}, Q_{\overline{0}}, P_{1}, Q_{2}$ are all nonzero on an open subset $Z^{0} \subset \mathcal{Z}$ which is surjective for the projection $\mathcal{Z} \rightarrow \mathcal{B}$. Since normality requires that these functions be generically nonzero, we further restrict $\mathcal{W}$ to this open subset.

Let $\overline{\mathcal{W}}$ denote the pullback of $\mathcal{W}$ to $z^{0} ; \overline{\mathcal{W}}$ is a rank 6 Pfaffian EDS on $z^{0}$. Because differentiating the equations defining $z$ yields no new relations, $\overline{\mathcal{W}}$ is torsionfree; moreover, it is straightforward to check that $\overline{\mathcal{W}}$ is involutive with last nonzero Cartan character $s_{1}=2$. (See [8] for a discussion of Cartan's test and involutivity.) 
Therefore, it follows from the Cartan-Kähler Theorem that local integral manifolds exist and are parametrized by 2 functions of one variable.

We summarize this result as:

Proposition 4.3. Let $(\mathcal{M}, \mathcal{I})$ be a hyperbolic Monge-Ampère system which is not Monge-integrable, and which is Darboux-integrable after one prolongation. Then near any point $p \in \mathcal{M}$ there is an open set $U \subset \mathcal{M}$ around $p$ such that the restriction of $\mathcal{I}$ to $U$ is linked to the wave equation by a normal Bäcklund transformation; moreover, the set of all such Bäcklund transformations is parametrized by 2 functions of one variable. Up to contact transformations, all such Bäcklund transformations preserve the space of independent variables $x, y$.

4.2. The Monge-integrable case. In this subsection $(\mathcal{M}, \mathcal{I})$ is assumed to be a hyperbolic Monge-Ampère system which is Monge-integrable and Darboux-integrable after one prolongation. As explained below, we will construct a canonical coframing associated to the partial prolongation of $\mathcal{I}$, and then proceed as in $\S 4.1$.

A similar argument to that of Lemma 4.1 can be used to prove:

Lemma 4.4. Near any point of $\mathcal{N}$, there exists a coframing $\left(\theta, \pi_{1}, \pi_{2}, \eta^{1}, \eta^{2}\right)$ such that $\theta$ spans the 1 -forms of $\mathcal{I}$, and the characteristic systems $\mathcal{C}_{1}, \mathcal{C}_{2}$ of $\mathcal{I}$ have derived flags

$$
\mathcal{C}_{1}=\left\{\theta, \pi_{1}, \eta^{1}\right\} \supset\left\{\pi_{1}, \eta^{1}\right\} \supset\left\{\eta^{1}\right\}=\mathcal{C}_{1}^{(\infty)}, \quad \mathcal{C}_{2}=\left\{\theta, \pi_{2}, \eta^{2}\right\} \supset\left\{\pi_{2}, \eta^{2}\right\}=\mathcal{C}_{2}^{(\infty)} .
$$

Indeed, the same coframing as that given in the proof of Lemma 4.1 satisfies the conditions of Lemma 4.4. Note that this lemma only assumes the Monge-integrability of $(\mathcal{M}, \mathcal{I})$.

In terms of the local coframing on $\mathcal{M}$ given by the lemma, the partial prolongation $\left(\mathcal{M}^{\prime}, \mathcal{I}^{\prime}\right)$ is defined as follows: let $\mathcal{M}^{\prime}=\mathcal{M} \times \mathbb{R}$, with coordinate $r$ on the $\mathbb{R}$ factor, and let $\mathcal{I}^{\prime}$ be the Pfaffian system on $\mathcal{M}^{\prime}$ generated by $\theta$, the 1 -form $\theta_{1}=\pi_{1}-r \eta^{1}$, and the 2 -form $\pi_{2} \wedge \eta^{2}$.

Lemma 4.5. Near any point of $\mathcal{M}^{\prime}$ there exists a coframing $\left(\theta, \theta_{1}, \pi_{2}, \eta^{1}, \eta^{2}, \pi_{3}\right)$ such that $\mathcal{I}^{\prime}$ is generated by $\theta, \theta_{1}$, and $\pi_{2} \wedge \eta^{2}$, satisfying

$$
\begin{aligned}
d \theta & =-\theta_{1} \wedge \eta^{1}-\theta_{2} \wedge \eta^{2} \quad \bmod \theta \\
d \theta_{1} & =-\pi_{3} \wedge \eta^{1} \bmod \theta, \theta_{1},
\end{aligned}
$$

with the derived flags of the characteristic systems of $\mathcal{I}^{\prime}$ given by

$$
\begin{aligned}
& \mathcal{C}_{1}^{\prime}=\left\{\theta, \theta_{1}, \eta^{1}, \pi_{3}\right\} \supset\left\{\theta_{1}, \eta^{1}, \pi_{3}\right\} \supset\left\{\eta^{1}, \pi_{3}\right\}=\mathcal{C}_{1}^{\prime(\infty)}, \\
& \mathcal{C}_{2}^{\prime}=\left\{\theta, \theta_{1}, \pi_{2}, \eta^{2}\right\} \supset\left\{\theta, \pi_{2}, \eta^{2}\right\} \supset\left\{\pi_{2}, \eta^{2}\right\}=\mathcal{C}_{2}^{\prime(\infty)} .
\end{aligned}
$$

Proof. As in the proof of Lemma 4.2, the usual or "full" prolongation of $\mathcal{I}$ on $\mathcal{M} \times \mathbb{R}^{2}$ is generated by $\theta, \theta_{1}$ and $\theta_{2}=\pi_{2}-t \eta^{2}$. We may construct the coframing $\left(\theta, \theta_{1}, \theta_{2}, \eta^{1}, \eta^{2}, \pi_{3}, \pi_{4}\right)$ on $\mathcal{M} \times \mathbb{R}^{2}$ precisely as in Lemma 4.2 , and this coframing satisfies the structure equations (4.2).

The hypothesis of Darboux-integrability implies that the characteristic system

$$
\mathcal{K}_{1}=\left\{\theta, \theta_{1}, \theta_{2}, \eta_{1}, \pi_{3}\right\}
$$


of the prolongation contains a rank 2 Frobenius system. As in Lemma 4.2, it follows from the structure equations and the construction of $\pi_{3}$ that

$$
\mathcal{K}_{1}^{(1)}=\left\{\theta, \theta_{1}, \eta_{1}, \pi_{3}\right\}
$$

However, this system is well-defined on $\mathcal{M}^{\prime}=\mathcal{M} \times \mathbb{R}$, and it coincides with the characteristic system $\mathcal{C}_{1}^{\prime}$ given in the statement of the present Lemma. It follows that we may adjust $\pi_{3}$ so that it lies in the rank 2 Frobenius system $\mathcal{C}_{1}^{\prime(\infty)}$.

Note that the second characteristic system $\mathcal{C}_{2}^{\prime}$ is simply the sum of $\mathcal{I}^{\prime}$ and the pullback of the characteristic system $\mathcal{C}_{2}$ of $\mathcal{I}$, and the structure of its derived flag follows from that of $\mathcal{C}_{2}$. $\square$

The conditions in Lemma 4.5 are preserved by changes of coframing of the form

$$
\left[\begin{array}{c}
\tilde{\theta} \\
\tilde{\theta}_{1} \\
\tilde{\pi}_{2} \\
\tilde{\eta}^{1} \\
\tilde{\eta}^{2} \\
\tilde{\pi}_{3}
\end{array}\right]=\left[\begin{array}{cccccc}
c & 0 & 0 & 0 & 0 & 0 \\
0 & a_{1} c & 0 & 0 & 0 & 0 \\
0 & 0 & a_{2} c & 0 & b_{1} & 0 \\
0 & 0 & 0 & a_{1}^{-1} & 0 & 0 \\
0 & 0 & b_{2} & 0 & a_{2}^{-1} & 0 \\
0 & 0 & 0 & b_{3} & 0 & a_{1}^{2} c
\end{array}\right]^{-1}\left[\begin{array}{c}
\theta \\
\theta_{1} \\
\pi_{2} \\
\eta^{1} \\
\eta^{2} \\
\pi_{3}
\end{array}\right]
$$

with $a_{1}, a_{2}, c \neq 0$. Let $G \subset G L(6, \mathbb{R})$ be the group of such transformations, and let $\mathcal{P}$ be the $G$-structure on $\mathcal{M}^{\prime}$ of which the coframing of Lemma 4.5 is a section.

After absorbing as much torsion as possible and differentiating to uncover relations among the torsion, $\mathcal{P}$ has structure equations

$$
\left[\begin{array}{l}
d \theta \\
d \theta_{1} \\
d \pi_{2} \\
d \eta^{1} \\
d \eta^{2} \\
d \pi_{3}
\end{array}\right]=-\left[\begin{array}{cccccc}
\gamma & 0 & 0 & 0 & 0 & 0 \\
0 & \gamma+\alpha_{1} & 0 & 0 & 0 & 0 \\
0 & 0 & \gamma+\alpha_{2} & 0 & \beta_{1} & 0 \\
0 & 0 & 0 & -\alpha_{1} & 0 & 0 \\
0 & 0 & \beta_{2} & 0 & -\alpha_{2} & 0 \\
0 & 0 & 0 & \beta_{3} & 0 & \gamma+2 \alpha_{1}
\end{array}\right] \wedge\left[\begin{array}{c}
\theta \\
\theta_{1} \\
\pi_{2} \\
\eta^{1} \\
\eta^{2} \\
\pi_{3}
\end{array}\right]
$$

$$
-\left[\begin{array}{c}
\theta_{1} \wedge \eta^{1}+\pi_{2} \wedge \eta^{2} \\
\pi_{3} \wedge \eta^{1}+\left(A \pi_{2}+B \eta^{2}\right) \wedge \theta \\
0 \\
0 \\
0 \\
C \pi_{3} \wedge \theta_{1}
\end{array}\right]
$$

Because of the dimensions of the derived flags of the characteristic systems (given in Lemma 4.5), $A, B$ are not both zero. Furthermore, we can choose a local section 
$\sigma: \mathcal{N}^{\prime} \rightarrow \mathcal{P}$ satisfying the conditions that $\eta^{1}=d x, \eta^{2}=d y, \pi_{2}$ is exact, and $\pi_{3}$ is integrable. The resulting coframing is uniquely determined.

When we pull back the structure equations via $\sigma$, the pseudoconnection forms $\alpha_{1}, \alpha_{2}, \beta_{1}, \beta_{2}, \beta_{3}, \gamma$ become semi-basic. By making use of the remaining ambiguity in these forms and the conditions imposed thus far on the coframing, we can assume that

$$
\alpha_{1}=E \eta^{1}, \quad \alpha_{2}=0, \quad \beta_{1}=G \pi_{2}, \quad \beta_{2}=0, \quad \beta_{3}=(-H+2 E) \pi_{3}, \quad \gamma=F \pi_{2}+G \eta^{2}
$$

for some functions $E, F, G, H$. Then the structure equations for this coframing become:

$$
\begin{aligned}
d \theta & =\theta \wedge\left(F \pi_{2}+G \eta^{2}\right)-\theta_{1} \wedge \eta^{1}-\pi_{2} \wedge \eta^{2} \\
d \theta_{1} & =\theta_{1} \wedge\left(F \pi_{2}+E \eta^{1}+G \eta^{2}\right)-\pi_{3} \wedge \eta^{1}+\theta \wedge\left(A \pi_{2}+B \eta^{2}\right) \\
d \pi_{2} & =0 \\
d \eta^{1} & =0 \\
d \eta^{2} & =0 \\
d \pi_{3} & =\pi_{3} \wedge\left(-C \theta_{1}+F \pi_{2}+H \eta^{1}+G \eta^{2}\right) .
\end{aligned}
$$

Once again, we will need to compute relations among the derivatives of the torsion functions in order to show that $(\mathcal{M}, \mathcal{I})$ has a Bäcklund transformation to the wave equation. We begin by differentiating the structure equations (4.34). We denote derivatives as, e.g.,

$$
d A=A_{0} \theta+A_{1} \theta_{1}+A_{2} \pi_{2}+A_{3} \eta^{1}+A_{4} \eta^{2}+A_{5} \pi_{3} .
$$

Computing $d(d \theta)=d\left(d \theta_{1}\right)=d\left(d \pi_{3}\right)=0$ yields the following equations for the derivatives of the torsion functions:

$$
\begin{aligned}
& d A=A_{0} \theta+A C \theta_{1}+A_{2} \pi_{2}-A E \eta^{1}+A_{4} \eta^{2} \\
& d B=B_{0} \theta+B C \theta_{1}+A_{4} \pi_{2}-B E \eta^{1}+B_{4} \eta^{2} \\
& d C=C_{1} \theta_{1}+C F \pi_{2}+C_{3} \eta^{1}+C G \eta^{2}+C_{5} \pi_{3} \\
& d E=E_{1} \theta_{1}+2 A \pi_{2}+E_{3} \eta^{1}+2 B \eta^{2}-C \pi_{3} \\
& d F=A C \theta+F_{2} \pi_{2}+A \eta^{1}+F_{4} \eta^{2} \\
& d G=B C \theta+F_{4} \pi_{2}+B \eta^{1}+G_{4} \eta^{2} \\
& d H=\left(C E-C_{3}\right) \theta_{1}+A \pi_{2}+H_{3} \eta^{1}+B \eta^{2}+H_{5} \pi_{3} .
\end{aligned}
$$

We may obtain further relations among the derivatives of the torsion functions by differentiating equations (4.35). Computing $d(d F) \equiv d(d G) \equiv 0$ modulo $\pi_{2}, \eta^{2}$ and recalling that $A, B$ cannot vanish simultaneously yields

$$
A_{0}=A\left(C_{3}-C E\right), \quad B_{0}=B\left(C_{3}-C E\right), \quad C_{1}=-C^{2}, \quad C_{5}=0 .
$$

Then computing $d(d A) \equiv d(d B) \equiv 0$ modulo $\theta, \pi_{2}, \eta^{2}$ yields

$$
E_{1}=2\left(C E-C_{3}\right)
$$

and $d(d C)=0$ implies that

$$
d C_{3}=\left(C^{2} E-2 C C_{3}\right) \theta_{1}+\left(A C+F C_{3}\right) \pi_{2}+C_{33} \eta^{1}+\left(B C+G C_{3}\right) \eta^{2}-C^{2} \pi_{3} .
$$


Finally, computing $d(d A) \equiv d(d B) \equiv 0$ modulo $\pi_{2}, \eta^{2}$ yields

$$
C_{33}=C E_{3}+E C_{3} .
$$

We now have all the relations that will be needed for the involutivity calculation below.

Now suppose that $\overline{\mathcal{M}}=\mathbb{R}^{5}$ carries a Monge-Ampère system $\overline{\mathcal{I}}$ representing the wave equation $Z_{X Y}=0$, generated algebraically by the contact form

$$
\bar{\theta}=d Z-P d X-Q d Y
$$

and the 2-forms $d P \wedge d X$ and $d Q \wedge d Y$. As in $\S 4.1$, if there were a Bäcklund transformation $\mathcal{B} \subset \mathcal{M} \times \overline{\mathcal{M}}$, then $Z$ would be a local coordinate on the fibers of $\mathcal{B} \rightarrow \mathcal{M}$ and the functions $X, Y, P, Q$ on $\mathcal{B}$ would satisfy the Bäcklund condition

$$
\{d P \wedge d X, d Q \wedge d Y\} \equiv\left\{\pi_{1} \wedge \eta^{1}, \pi_{2} \wedge \eta^{2}\right\} \bmod \theta, \bar{\theta}
$$

As in $\S 4.1$, let $\mathcal{B}^{\prime}=\mathcal{M}^{\prime} \times \mathbb{R}$, again with $Z$ as the coordinate on the second factor; we extend the projection $\mathcal{M}^{\prime} \rightarrow \mathcal{M}$ to a projection $\mathcal{B}^{\prime} \rightarrow \mathcal{B}$ by the identity on the second factor. We will regard $X, Y, P, Q$ as functions on $\mathcal{B}^{\prime}$, but require that

$$
d X, d Y, d P, d Q \in\left\{\theta, \bar{\theta}, \theta_{1}, \pi_{2}, \eta^{1}, \eta^{2}\right\}
$$

so that they are in fact well-defined on $\mathcal{B}$. In order to satisfy the Bäcklund condition (4.37), we will furthermore require that

$$
\{d X, d P\} \subset\left\{\theta, \bar{\theta}, \theta_{1}, \eta^{1}\right\}, \quad\{d Y, d Q\} \subset\left\{\theta, \bar{\theta}, \pi_{2}, \eta^{2}\right\} .
$$

The same argument as that given in $\S 4.1$ shows that by a contact transformation on $\overline{\mathcal{M}}$, we may assume that $X=x$. However, the same is not true for $Y$ : the system $\left\{\theta, \bar{\theta}, \pi_{2}, \eta^{2}\right\}$ on $\mathcal{B}$ contains a rank 3 integrable subsystem, so we cannot necessarily arrange to have $\eta^{2} \in\{d Y, d Q\}$. There are three different, geometrically natural conditions that we could impose on the intersection of the rank 2 Pfaffian systems $\{d Y, d Q\}$ and $\left\{\pi_{2}, \eta^{2}\right\}$, each of them potentially leading to a different type of Bäcklund transformation:

1. $\{d Y, d Q\} \cap\left\{\pi_{2}, \eta^{2}\right\}$ has rank 1 and is spanned by a non-integrable 1-form.

2. $\{d Y, d Q\} \cap\left\{\pi_{2}, \eta^{2}\right\}$ has rank 1 and is spanned by an integrable 1 -form.

3. $\{d Y, d Q\} \cap\left\{\pi_{2}, \eta^{2}\right\}$ has rank 2 .

In cases (2) and (3) we can arrange that $Y=y$ via contact transformations on $\mathcal{M}$ and $\overline{\mathcal{M}}$, but in case (1) this is not possible.

4.2.1. Case (1). In this case we have

$$
\bar{\theta}=d Z-P d x-Q d Y,
$$

and condition (4.38) becomes

$$
d P \in\left\{\theta, \bar{\theta}, \theta_{1}, \eta^{1}\right\}, \quad d Y, d Q \in\left\{\theta, \bar{\theta}, \pi_{2}, \eta^{2}\right\} .
$$

Suppose that

$$
\begin{aligned}
& d P=P_{0} \theta+P_{\overline{0}} \bar{\theta}+P_{1} \theta_{1}+P_{3} \eta^{1} \\
& d Q=Q_{0} \theta+Q_{\overline{0}} \bar{\theta}+Q_{2} \pi_{2}+Q_{4} \eta^{2} \\
& d Y=Y_{0} \theta+Y_{\overline{0}} \bar{\theta}+Y_{2} \pi_{2}+Y_{4} \eta^{2} .
\end{aligned}
$$


Normality of the Bäcklund transformation requires that $P_{1} \neq 0, Q_{2} Y_{4}-Q_{4} Y_{2} \neq 0$, and $P_{1} \neq Q_{2} Y_{4}-Q_{4} Y_{2}$.

The argument proceeds in much the same fashion as that of $\S 4.1$ : differentiating equations (4.39) leads to relations among the derivatives of $P, Q, Y$. Eventually we are led to a Pfaffian exterior differential system $\mathcal{W}$ whose integral manifolds satisfying the independence condition $\theta \wedge \bar{\theta} \wedge \theta_{1} \wedge \pi_{2} \wedge \eta^{1} \wedge \eta^{2} \neq 0$ are in one-to-one correspondence with the desired functions $P, Q, Y$ defining a Bäcklund transformation. This EDS is involutive with last nonzero Cartan character $s_{3}=1$. Therefore, local integral manifolds exist and are parametrized by 1 function of three variables.

If we impose the additional condition that the Bäcklund transformation be holonomic, we find that the resulting EDS is involutive with last nonzero Cartan character $s_{2}=2$. Therefore, among the Bäcklund transformations of this type, there is a small, proper subset, parametrized by 2 functions of two variables, consisting of holonomic transformations.

4.2.2. Case (2). In this case, we can use contact transformations on $\mathcal{M}$ and $\overline{\mathcal{M}}$ to arrange that $\{d Y, d Q\} \cap\left\{\pi_{2}, \eta^{2}\right\}$ is spanned by $\eta^{2}=d y=d Y$. Then we have

$$
\bar{\theta}=d Z-P d x-Q d y,
$$

and condition (4.38) becomes

$$
d P \in\left\{\theta, \bar{\theta}, \theta_{1}, \eta^{1}\right\}, \quad d Q \in\left\{\theta, \bar{\theta}, \pi_{2}, \eta^{2}\right\} .
$$

Suppose that

$$
\begin{aligned}
& d P=P_{0} \theta+P_{\overline{0}} \bar{\theta}+P_{1} \theta_{1}+P_{3} \eta^{1}, \\
& d Q=Q_{0} \theta+Q_{\overline{0}} \bar{\theta}+Q_{2} \pi_{2}+Q_{4} \eta^{2} .
\end{aligned}
$$

Normality of the Bäcklund transformation requires that $P_{1}, Q_{2} \neq 0$ and $P_{1} \neq Q_{2}$.

Differentiating equations (4.40) leads to relations among the derivatives of $P, Q$, and to a Pfaffian exterior differential system $\mathcal{W}$ whose integral manifolds satisfying the independence condition $\theta \wedge \bar{\theta} \wedge \theta_{1} \wedge \pi_{2} \wedge \eta^{1} \wedge \eta^{2} \neq 0$ are in one-to-one correspondence with the desired functions $P, Q$ defining a Bäcklund transformation. This EDS is involutive with last nonzero Cartan character $s_{2}=1$. Therefore, local integral manifolds exist and are parametrized by 1 function of two variables.

If we impose the additional condition that the Bäcklund transformation be holonomic, we find that the resulting EDS is involutive with last nonzero Cartan character $s_{1}=3$. Therefore, among the Bäcklund transformations of this type, there is a small, proper subset, parametrized by 3 functions of one variable, consisting of holonomic transformations.

4.2.3. Case (3). In this case, we can use contact transformations on $\mathcal{M}$ and $\overline{\mathcal{M}}$ to arrange that $\eta^{2}=d y=d Y, \pi_{2}=d Q$. Then we have

$$
\bar{\theta}=d Z-P d x-Q d y,
$$

and condition (4.38) becomes

$$
d P \in\left\{\theta, \bar{\theta}, \theta_{1}, \eta^{1}\right\}
$$

Suppose that

$$
d P=P_{0} \theta+P_{\overline{0}} \bar{\theta}+P_{1} \theta_{1}+P_{3} \eta^{1} .
$$


Normality of the Bäcklund transformation requires that $P_{1} \neq 0$ and $P_{1} \neq 1$.

Differentiating equation (4.41) leads to relations among the derivatives of $P$, and to a Pfaffian exterior differential system $\mathcal{W}$ whose integral manifolds satisfying the independence condition $\theta \wedge \bar{\theta} \wedge \theta_{1} \wedge \pi_{2} \wedge \eta^{1} \wedge \eta^{2} \neq 0$ are in one-to-one correspondence with the desired functions $P$ defining a Bäcklund transformation.

The involutivity calculation in this case depends on the torsion functions in the structure equations (4.34). If

$$
A G-B F=A C+F\left(C E-C_{3}\right)=A F^{2}+F A_{2}-A F_{2}=B F^{2}+F A_{4}-A F_{4}=0,
$$

then $\mathcal{W}$ is involutive with last nonzero Cartan character $s_{2}=1$, and so local integral manifolds exist and are parametrized by 1 function of two variables. Otherwise, there are no solutions with $P_{1} \neq 0$, and hence no normal Bäcklund transformations of this type.

Observe that in this case, the $G$-structure on the Bäcklund transformation $\mathcal{B}$ (cf. $\S 2)$ will satisfy the condition that (omitting obvious pullback notations)

$$
\left\{\omega^{3}, \omega^{4}\right\}=\left\{\pi_{2}, \eta^{2}\right\}=\{d Y, d Q\} .
$$

Therefore, all transformations of this type satisfy the hypotheses of Proposition 3.4 and so are holonomic.

We summarize these results as:

Proposition 4.6. Let $(\mathcal{M}, \mathcal{I})$ be a hyperbolic Monge-Ampère system which is Monge-integrable, and Darboux-integrable after one prolongation. Then there exist Bäcklund transformations of types (1) and (2) above between $(\mathcal{M}, \mathcal{I})$ and the standard wave equation $(\overline{\mathcal{M}}, \overline{\mathcal{I}})$, and of type (3) if the torsion functions of $(\mathcal{M}, \mathcal{I})$ satisfy $(4.42)$. The generic Bäcklund transformation is of type (1) and does not preserve the space of independent variables. There are both holonomic and non-holonomic Bäcklund transformations of types (1) and (2), and all Bäcklund transformations of type (3) are holonomic.

5. Examples. In this section we review the classifications of second-order Darboux-integrable Monge-Ampère equations, due to Goursat and Vessiot, and discuss the connection between our results and the work of Zvyagin. We will also give examples of a method for explicitly solving for Bäcklund transformations linking these equations to the wave equation.

5.1. The Goursat-Vessiot List. Goursat [7] studied non-linear PDE of the form

$$
u_{x y}=F\left(x, y, u, u_{x}, u_{y}\right)
$$

which are Darboux-integrable at the 2-jet level, classifying them up to complex contact transformations that preserve the form (5.1). Using Lie-theoretic techniques, Vessiot [11] reproduced Goursat's classification, expanded to include linear equations, and showed that some of the equations on Goursat's list were equivalent under more general contact transformations. Recently, Biesecker [1] re-proved Vessiot's classification using Cartan's method of equivalence, with respect to real contact transformations. 
Retaining Goursat's numbering, the list is:

$$
\begin{aligned}
(x+y) u_{x y} & =2 \sqrt{u_{x} u_{y}} ; \\
u u_{x y} & =\sqrt{1+u_{x}^{2}} \sqrt{1+u_{y}^{2}} ; \\
(\sin u) u_{x y} & =\sqrt{1+u_{x}^{2}} \sqrt{1+u_{y}^{2}} ; \\
u u_{x y} & = \pm \phi\left(u_{x}\right) \psi\left(u_{y}\right),
\end{aligned}
$$

where $\phi(t), \psi(t)$ satisfy the ODE $d f / d t \pm t / f=K$ for some nonzero constant $K$;

$$
(x+y) u_{x y}=\gamma\left(u_{x}\right) \gamma\left(u_{y}\right),
$$

where $\gamma$ is implicitly defined by $\gamma(t)-1=\exp (t-\gamma(t))$;

$$
\begin{aligned}
u_{x}-u \frac{u_{x y}}{u_{y}} & =f\left(x, \frac{u_{x y}}{u_{y}}\right) ; \\
u_{x y} & =e^{u} \sqrt{1+\left(u_{x}\right)^{2}} ; \\
u_{x}-y u_{x y} & =f\left(x, u_{x y}\right) ; \\
u_{x y} & =e^{u} ; \\
u_{x y} & =u_{x} e^{u}, ; \\
u_{x y} & =\left(\frac{1}{u+x}+\frac{1}{u+y}\right) u_{x} u_{y} .
\end{aligned}
$$

(In (VI) and (VIII) the function $f$ is arbitrary.) To Goursat's original list, Vessiot added representatives of the two equivalence classes of Darboux-integrable linear equations:

$$
u_{x y}=a(x, y) u_{x}+b(x, y) u_{y}-a(x, y) b(x, y) u,
$$

where $h(x, y)=-a_{x}$ and $k(x, y)=-b_{y}$ must satisfy the system $(\ln h)_{x y}=2 h-k$, $(\ln k)_{x y}=2 k-h$ with $h \neq k$; and finally,

$$
u_{x y}=\frac{2 u}{(x+y)^{2}} \text {. }
$$

In the above list, we have replaced Goursat's original versions of (VII) and (XI) by simpler equations that Vessiot showed were equivalent to them by contact transformations; see [11], part 2, pages 5 and 6 , respectively. Vessiot also observed that (VI) is contact-equivalent to (X), and (VIII) may be reduced by a contact transformation to the special case

$$
u_{x y}=\frac{u_{x}}{x+y}
$$

Our Theorem 1, together with Goursat's classification, implies the following

Corollary 3. If a second-order Monge-Ampère PDE for one function of two variables is linked to the standard wave equation by a normal Bäcklund transformation with 1-dimensional fibers, then the PDE is either equivalent to the wave equation by a contact transformation, or equivalent to one of the equations (I)-(XIII) in the above list. 
5.2. Zvyagin's List. Zvyagin [14] investigated second-order Monge-Ampère equations linked to the standard wave equation by a Bäcklund transformation, and asserted that all such transformations that are non-holonomic are exhausted by a list of six examples in addition to Liouville's equation. Zvyagin did not publish a proof of this classification, and did not give explicit forms for the Monge-Ampère equations for some of the transformations on his list. He did give an explicit transformation for Goursat-Vessiot equation (I):

$$
\sqrt{p}-\sqrt{P}=\sqrt{\frac{Z-u}{x-y}}, \quad \sqrt{q}-\sqrt{Q}=-\sqrt{\frac{Z-u}{x-y}},
$$

where, as in $\S 4, Z$ is the solution to the wave equation, with $x$ - and $y$-derivatives $P$ and $Q$. (The $x$ - and $y$-coordinates are preserved by the transformation.)

Corollary 3 implies that every one of Zvyagin's transformations must be identifiable with an equation on the Goursat-Vessiot list. We have calculated explicit forms for certain transformations on Zvyagin's list, and we can identify the following transformations as belonging to equations (II), (III), and (VII), respectively:

$$
\begin{gathered}
Z P-u p=\sqrt{Z^{2}-u^{2}} \sqrt{1+P^{2}}, \quad Z Q-u q=\sqrt{Z^{2}-u^{2}} \sqrt{1+Q^{2}} ; \\
p=\frac{\left(\sinh Z+\frac{1}{2} e^{-w}\right) P+e^{(Z-w) / 2} \sqrt{P^{2}-1}}{-\sin u}, \\
q=\frac{\left(\sinh Z-\frac{1}{2} e^{-w}\right) Q+e^{-(w+Z) / 2} \sqrt{Q^{2}-1}}{-\sin u},
\end{gathered}
$$

where $w$ is related to $u$ and $Z$ by $\cos u=\cosh Z-\frac{1}{2} e^{-w}$;

$$
\begin{gathered}
p=\left(1-2 e^{u+Z}\right) P-2 e^{(u+Z) / 2} \sqrt{e^{u+Z}-1} \sqrt{P^{2}+1}, \\
q=-Q-e^{(u-Z) / 2} \sqrt{e^{u+Z}-1} .
\end{gathered}
$$

Each of the above transformations, which preserve the $x$ - and $y$-coordinates, may be verified as being non-holonomic. To see how this is done, suppose that the transformation equations, when solved for $p$ and $q$, take the form

$$
p=f(x, y, u, Z, P), \quad q=g(x, y, u, Z, Q) .
$$

The Cartan system for the Monge-Ampère equation is spanned by $d x, d y, d u, d p$ and $d q$, while the Cartan system for the wave equation is spanned by $d x, d y, d Z, d P, d Q$. Recall from $\S 2$ that a transformation is holonomic if the intersection of these systems is Frobenius. In light of the transformation equations (5.2), the intersection of these two systems is spanned by $d x, d y, d p-f_{u} d u$ and $d q-g_{u} d u$. The last two 1-forms are congruent modulo $d x$ and $d y$ to

$$
\xi_{1}=f_{P} d P+f_{Z} d Z, \quad \xi_{2}=g_{Q} d Q+g_{Z} d Z,
$$

respectively. To check that the system $\left\{d x, d y, \xi_{1}, \xi_{2}\right\}$ is not Frobenius, compute

$$
\begin{aligned}
& d \xi_{1} \wedge \xi_{1} \wedge \xi_{2} \equiv g_{Q}\left(f_{u Z} f_{P}-f_{P u} f_{Z}\right) d P \wedge d Q \wedge d u \wedge d Z, \quad \bmod d x, d y \\
& d \xi_{2} \wedge \xi_{1} \wedge \xi_{2} \equiv f_{P}\left(g_{u Z} g_{Q}-g_{Q u} g_{Z}\right) d P \wedge d Q \wedge d u \wedge d Z
\end{aligned}
$$

In each case, the coefficients on the right are nonzero, and we conclude that the transformation is non-holonomic. 
5.3. Solving for Bäcklund transformations. In this subsection, we will set up systems of PDE whose solutions are Bäcklund transformations to the wave equation for some examples on the Goursat-Vessiot list. Although the existence of these transformations follows from the arguments of $\S 4$, here we will be able to go further in writing down explicit formulas for the transformations. Because we will work with specific Monge-Ampère equations on the list, we can take advantage of explicit formulas for the characteristic invariants. (These invariants are computed, for example, in the dissertation of M. Biesecker [1].)

The general approach is as follows. We write a PDE on the list in the form

$$
s=F(x, y, u, p, q)
$$

This form always has $x$ and $y$ as characteristic invariants, and we assume these are the only functionally independent invariants up to first order for the equation (i.e., we assume that the equation is not Monge-integrable). The Bäcklund transformation must take these invariants to corresponding characteristic invariants for the wave equation. By employing a change of variables on the wave equation side, of the form $X \mapsto \phi(X), Y \mapsto \psi(Y)$, and interchanging $X$ and $Y$ if necessary, we may assume that the transformation has

$$
x=X, \quad y=Y .
$$

Now suppose that the remaining equations defining the Bäcklund transformation take the form

$$
p=f(x, y, u, Z, P, Q), \quad q=g(x, y, u, Z, P, Q) .
$$

The Monge-Ampère system on $\mathbb{R}^{5}$ encoding the PDE (5.4) is generated algebraically by the contact form $\theta=d u-p d x-q d y$ and the 2 -forms

$$
\Omega_{1}=(d p-F(x, y, u, p, q) d y) \wedge d x, \quad \Omega_{2}=(d q-F(x, y, u, p, q) d x) \wedge d y .
$$

The defining property of the Bäcklund transformation is that substituting (5.5) into $\Omega_{1}, \Omega_{2}$ must make them congruent to linear combinations of $d P \wedge d x$ and $d Q \wedge d y$ (the 2 -forms defining the Monge-Ampère system for the wave equation) modulo $\theta$ and the contact form on the wave equation side,

$$
\bar{\theta}=d Z-P d x-Q d y .
$$

In fact, $\Omega_{1}$ must become congruent to a multiple of $d P \wedge d x$ and $\Omega_{2}$ congruent to a multiple of $d Q \wedge d y$. Using (5.5), we compute

$$
\Omega_{1} \equiv\left(\left(f_{y}+f_{u} g+f_{Z} Q-F\right) d y+f_{P} d P+f_{Q} d Q\right) \wedge d x \quad \bmod \theta, \bar{\theta},
$$

and

$$
\Omega_{2} \equiv\left(\left(g_{x}+g_{u} f+g_{Z} P-F\right) d x+g_{P} d P+g_{Q} d Q\right) \wedge d y \quad \bmod \theta, \bar{\theta} .
$$

We immediately conclude that $f_{Q}=g_{P}=0$, so that the transformation is of the form

$$
p=f(x, y, u, Z, P), \quad q=g(x, y, u, Z, Q),
$$


and $f, g$ must satisfy two additional first-order PDEs,

$$
\begin{aligned}
& f_{y}=F(x, y, u, f, g)-f_{u} g-f_{Z} Q, \\
& g_{x}=F(x, y, u, f, g)-g_{u} f-g_{Z} P .
\end{aligned}
$$

We derive additional first- and second-order PDEs that $f$ and $g$ must satisfy by differentiating the conditions so far. Taking derivatives with respect to $Q$ in (5.7) and $P$ in (5.8) gives

$$
f_{Z}=\left(F_{q}-f_{u}\right) g_{Q}, \quad g_{Z}=\left(F_{p}-g_{u}\right) f_{P},
$$

where the partials $F_{p}=\partial F / \partial p$ and $F_{q}=\partial F / \partial q$ are taken and then evaluated with $p$ and $q$ given by (5.6). As we will see in specific cases below, this will sometimes imply that $f$ and $g$ must be linear in $P$ and $Q$.

In what follows, let $J_{1}$ and $J_{2}$ denote the second-order characteristic invariants for the given PDE (whose existence makes the equation Darboux-integrable), expressed in terms of $x, y, u, p, q$ and the second-order jet coordinates $r$ and $t$. (We make the convention that $J_{1}$ is invariant along the characteristic curves where $x$ is constant, and $J_{2}$ is invariant when $y$ is constant.) Then the Bäcklund transformation must take $J_{1}$ and $J_{2}$ to second-order characteristic invariants for the wave equation. In order to compute these additional constraints, we must take total $x$ - and $y$-derivatives in (5.6) to deduce how the second-order jet coordinates $r$ and $t$ transform in terms of those of the wave equation:

$$
r=f_{x}+f_{u} f+f_{Z} P+f_{P} R, \quad t=g_{y}+g_{u} g+g_{Z} Q+g_{Q} T .
$$

Requiring that, under these substitutions, $J_{1}$ transforms to be a function of only $x, P, R$, and $J_{2}$ transforms to be a function of only $y, Q, T$, will lead to additional second-order PDEs which $f$ and $g$ must satisfy.

We now turn to specific examples.

Equation IX (Liouville's equation). In this case, $F=e^{u}$, and the equations (5.7) through (5.9) become

$$
\begin{aligned}
& f_{y}=e^{u}+f_{u}\left(Q g_{Q}-g\right), \\
& g_{x}=e^{u}+g_{u}\left(P f_{P}-f\right), \\
& f_{Z}=-f_{u} g_{Q} \\
& g_{Z}=-g_{u} f_{P} .
\end{aligned}
$$

As mentioned in $\S 1$, the characteristic invariants are

$$
J_{1}=r-\frac{1}{2} p^{2}, \quad J_{2}=t-\frac{1}{2} q^{2} .
$$

Under (5.6), the first invariant transforms as

$$
r-\frac{1}{2} p^{2}=f_{x}+f_{u} f+f_{Z} P+f_{P} R-\frac{1}{2} f^{2} .
$$

Requiring that this be a function of $x, P, R$ only immediately implies that $f_{P}$ can depend on $x$ and $P$ only, and that the remaining terms have no dependence on $u, Z$ or $y$. This gives us 6 additional second-order PDEs for $f$ :

$$
f_{P u}=f_{P y}=f_{P Z}=0, \quad \partial_{u}, \partial_{y}, \partial_{Z}\left(f_{x}+f_{u} f+f_{Z} P-\frac{1}{2} f^{2}\right)=0 .
$$


Similarly, we also get

$$
g_{Q u}=g_{Q y}=g_{Q Z}=0, \quad \partial_{u}, \partial_{x}, \partial_{Z}\left(g_{y}+g_{u} g+g_{Z} Q-\frac{1}{2} g^{2}\right)=0 .
$$

Note that some of these second-order equations are redundant, in light of the derivatives of (5.13) and (5.14).

Next, we derive additional equations by differentiation. Note that (5.11) shows that $f_{u}$ cannot be identically zero; then, taking a $Q$ derivative of (5.13) shows that $g_{Q Q}=0$. Similarly, $f_{P P}=0$, so that $f$ and $g$ are linear in $P$ and $Q$. Thus, we may set

$f(x, y, u, P, Z)=f^{0}(x) P+f^{1}(x, y, u, Z), \quad g(x, y, u, Q, Z)=g^{0}(y) Q+g^{1}(x, y, u, Z)$.

In particular, taking the terms in (5.15), (5.16) that are linear in $P$ and $Q$ respectively gives

$$
\partial_{u}\left(\left(f^{0}-g^{0}\right) f_{u}^{1}-f^{0} f^{1}\right)=0, \quad \partial_{u}\left(\left(g^{0}-f^{0}\right) g_{u}^{1}-g^{0} g^{1}\right)=0 .
$$

Furthermore, equating the $Z$-derivative of (5.11) with the $y$-derivative of (5.13), and using the $u$-derivatives of these equations to determine $f_{y u}$ and $f_{Z u}$, gives the compatibility condition

$$
\left(g^{0}-f^{0}\right) f_{u}^{1} g_{u}^{1}=g_{y}^{0} f_{u}^{1}+e^{u} g^{0}
$$

we similarly derive

$$
\left(f^{0}-g^{0}\right) f_{u}^{1} g_{u}^{1}=f_{x}^{0} g_{u}^{1}+e^{u} f^{0} .
$$

Adding and differentiating with respect to $u$, and using the values for $f_{u u}^{1}$ and $g_{u u}^{1}$ given by (5.17), shows that $f^{0}=-g^{0}=k$ for some nonzero constant $k$, and $f_{u}^{1} g_{u}^{1}=$ $\frac{1}{2} e^{u}$. Integrating the remaining equations shows that the most general form for the transformation is

$$
\begin{aligned}
& p=k P+2 \exp \left(\frac{u+k Z+v(x)+w(y)}{2}\right)+v^{\prime}(x), \\
& q=-k Q+\exp \left(\frac{u-k Z-v(x)-w(y)}{2}\right)-w^{\prime}(y),
\end{aligned}
$$

where $v(x), w(y)$ are arbitrary functions.

Using the calculation (5.3), it is easy to verify that none of these transformations is holonomic.

In the next two examples, we will analyze the system of PDEs that $f$ and $g$ must satisfy using the techniques of exterior differential systems.

Equation XIII. This PDE,

$$
s=F(u, x, y):=\frac{2 u}{(x+y)^{2}},
$$

has second-order characteristic invariants

$$
J_{1}=r+\frac{2 p}{x+y}, \quad J_{2}=t+\frac{2 q}{x+y}
$$


in the $x$ - and $y$-directions respectively (see [1], Appendix A). Substituting for $p$ and $r$ from (5.6) and (5.10) yields

$$
J_{1}=\left(f_{x}+f_{u} f+f_{Z} P+\frac{2 f}{x+y}\right)+f_{P} R
$$

so that $f_{P}$ and the expression in parentheses must be functions of $x$ and $P$ only. Similarly, we have

$$
J_{2}=\left(g_{y}+g_{u} g+g_{Z} Q+\frac{2 g}{x+y}\right)+g_{Q} T
$$

hence $g_{Q}$ and the expression in parentheses must be functions of $y$ and $Q$ only.

In this case, (5.7) through (5.9) specialize to

$$
\begin{aligned}
f_{y} & =F-\left(g-Q g_{Q}\right) f_{u}, \\
g_{x} & =F-\left(f-P f_{P}\right) g_{u}, \\
f_{Z} & =-g_{Q} f_{u}, \\
g_{Z} & =-f_{P} g_{u} .
\end{aligned}
$$

If $f_{u}$ were identically zero, then $f_{Z}$ would also be identically zero, but then $f_{y}=F=$ $2 u /(x+y)^{2}$ would give a contradiction. So, we may assume that $f_{u}$ and (similarly) $g_{u}$ are nonzero on an open dense set. It then follows from (5.21) that $g_{Q Q}=0$ and from (5.22) that $f_{P P}=0$, i.e., $f$ and $g$ are again linear in $P$ and $Q$.

Differentiating (5.20),(5.22) with respect to $x$ and $Z$, and equating mixed partials, enables us to solve for $f_{P x}$ as

$$
f_{P x}=f_{u}\left(f_{P}-g_{Q}\right)-\frac{2 f_{P}}{g_{u}(x+y)^{2}},
$$

while from (5.19),(5.21) we similarly obtain

$$
g_{Q y}=g_{u}\left(g_{Q}-f_{P}\right)-\frac{2 g_{Q}}{f_{u}(x+y)^{2}} .
$$

To encode the PDEs that $f$ and $g$ must satisfy as an exterior differential system, we will use $x, y, u, Z, P, Q$ as independent variables, and use $f, f_{x}, f_{u}, f_{P}, r_{1}$ and $g, g_{y}, g_{u}, g_{Q}, t_{1}$ as dependent variables. (The role of $r_{1}$ and $t_{1}$ will be made clear below.) We will regard these variables as coordinates on $\mathbb{R}^{16}$. As stated above, we restrict to the open subset $\mathcal{U} \subset \mathbb{R}^{16}$ where $f_{P}, f_{u}, g_{Q}$ and $g_{u}$ are nonzero.

The generator 1 -forms are $\psi_{1}$ through $\psi_{6}$, where

$$
\begin{aligned}
& \psi_{1}=-d f+f_{x} d x+f_{u} d u+f_{P} d P+f_{Z} d Z+f_{y} d y, \\
& \psi_{2}=-d g+g_{y} d y+g_{u} d u+g_{Q} d Q+g_{Z} d Z+g_{x} d x, \\
& \psi_{3}=-d f_{P}+f_{P x} d x \\
& \psi_{4}=-d g_{Q}+g_{Q y} d y
\end{aligned}
$$

with $f_{y}, f_{Z}, g_{x}, g_{Z}$ given by equations (5.21) through (5.20) and $f_{P x}, g_{Q y}$ given by (5.23) and (5.24). The remaining generators $\psi_{5}, \psi_{6}$ encode the rest of the condition 
that the second-order characteristic invariants be preserved. Differentiating the first term in $J_{1}$ gives

$$
\begin{aligned}
d\left(f_{x}+f_{z} P+f_{u} f+\right. & \left.\frac{2 f}{x+y}\right) \equiv d\left(f_{x}\right)+P d\left(f_{Z}\right)+\left(d\left(f_{u}\right)-\frac{2 d y}{(x+y)^{2}}\right) f \\
& +\left(f_{u}+\frac{2}{x+y}\right)\left(f_{u} \theta-g_{Q} f_{u} \bar{\theta}+F d y\right) \quad \bmod \psi_{1}, d x, d P .
\end{aligned}
$$

Let $\eta_{1}$ be the 1-form on the right; then for any Bäcklund transformation $\eta_{1}$ must be a linear combination of $d x$ and $d P$. In fact, since only the first term in $\eta_{1}$ can contain $d P$, the coefficient of $d P$ in $\eta_{1}$ must be $f_{P x}$. Thus, our remaining generators are

$$
\psi_{5}=\eta_{1}-f_{P x} d P-r_{1} d x, \quad \psi_{6}=\eta_{2}-g_{Q y} d Q-t_{1} d y,
$$

where, based a similar calculation of $d J_{2}$, we set

$\eta_{2}=d\left(g_{y}\right)+Q d\left(g_{Z}\right)+\left(d\left(g_{u}\right)-\frac{2 d x}{(x+y)^{2}}\right) g+\left(g_{u}+\frac{2}{x+y}\right)\left(g_{u} \theta-f_{P} g_{u} \bar{\theta}+F d x\right)$.

We seek to construct integral manifolds of the given differential ideal, i.e., submanifolds of $\mathcal{U}$ to which all the forms in the ideal pull back to be zero. An integral element for an EDS is an infinitesimal version of an integral manifold, i.e., a subspace in the tangent space to $\mathcal{U}$ at some point, to which all the forms in the ideal restrict to be zero. Because we want integral manifolds which are graphs of functions of $x, y, u, P, Q, Z$, we will only consider integral elements which are 6-dimensional, and to which the differentials $d x, d y, d u, d P, d Q, d Z$ restrict to be linearly independent; we will call these admissible integral elements.

Applying Cartan's Test to the Pfaffian system generated by $\psi_{1}, \ldots, \psi_{6}$ shows that it has last nonzero Cartan character $s_{1}=4$, but is not involutive, as the space of admissible integral elements has 2-dimensional fiber at each point. However, the system becomes involutive after one prolongation, and this establishes the existence of the required Bäcklund transformations. The last nonzero Cartan character of the involutive prolongation is $s_{1}=2$. By the Cartan-Kähler Theorem (see [8], Chapter 7) we conclude that 6-dimensional integral submanifolds, satisfying the independence condition, exist through every point of $\mathcal{U}$, and that the construction of such submanifolds depends on a choice of 2 functions of one variable.

The additional 1-forms that generate the prolongation include

$$
\begin{aligned}
& \psi_{7}=d\left(f_{u}\right)+\left(f_{u}^{2}+2 \frac{f_{u}}{x+y}\right) d x+\left(f_{u} g_{u}-\frac{2}{(x+y)^{2}}\right) d y, \\
& \psi_{8}=d\left(g_{u}\right)+\left(g_{u}^{2}+2 \frac{g_{u}}{x+y}\right) d y+\left(f_{u} g_{u}-\frac{2}{(x+y)^{2}}\right) d x,
\end{aligned}
$$

which are actually defined on the original manifold $\mathbb{R}^{16}$. These forms vanish on all integral elements of the original system, and if they had been included in the ideal, it would have been involutive with $s_{1}=2$.

The vanishing of $\psi_{7}, \psi_{8}$ implies that $f_{u}$ and $g_{u}$ are functions of $x$ and $y$ only. Moreover, forms $\psi_{7}, \psi_{8}$ define a smaller Pfaffian system, involving only $f_{u}, g_{u}$ as functions of $x$ and $y$, which satisfies the Frobenius condition. This means that $f_{u}(x, y)$ and $g_{u}(x, y)$ can be determined by solving systems of ODE. Once these are determined, substituting the solutions into (5.23) and (5.24) gives a Frobenius system which may 
be solved for the functions $f_{P}(x)$ and $g_{Q}(y)$. Then $f$ and $g$ may be determined by integrating first-order PDE, with $f$ including an arbitrary function of $x$ and $g$ an arbitrary function of $y$.

For example, by observing that $f_{u}+g_{u}$ must satisfy a Riccati equation as a function of $x+y$, we are led to a solution

$$
f_{u}=\frac{y}{(x+y) x}, \quad g_{u}=\frac{x}{(x+y) y} .
$$

Substituting these into $(5.23),(5.24)$ leads to $f_{P}+g_{Q}=k(x+y) /(x y)$ for a constant $k$. It is simplest to choose $k=0$ with $f_{P}=1$ and $g_{Q}=-1$. Integrating then gives the solution

$$
f=P+\frac{y(u+Z)}{x(x+y)}, \quad g=-Q+\frac{x(u-Z)}{y(x+y)} .
$$

Proposition 5.1. All Bäcklund transformations between (XIII) and the wave equation are holonomic.

Proof. As noted in $\S 5.2$, the holonomic condition is equivalent to the Pfaffian system on $\mathcal{B}^{6}$ spanned by $d x, d y$ and

$$
d p-f_{u} d u \equiv\left(-g_{Q} f_{u}\right) d Z+f_{P} d P, \quad d q-g_{u} d u \equiv\left(-f_{P} g_{u}\right) d Z+g_{Q} d Q, \quad \bmod d x, d y
$$

being Frobenius. It is straightforward to check that $d\left(f_{P} d P-g_{Q} f_{u} d Z\right)$ and $d\left(g_{Q} d Q-\right.$ $f_{P} g_{u} d Z$ ) are zero modulo $d x, d y$ and the 1-forms of the above EDS. (For example, $d\left(f_{P}\right) \equiv 0$ modulo $d x, d y, \psi_{1}, \ldots, \psi_{8}$, and the same is true for $d\left(g_{Q}\right)$ and $d\left(f_{u}\right)$.)

Equation IV. This PDE has the form

$$
s=F(u, p, q):= \pm \frac{\alpha(p) \beta(q)}{u},
$$

where $\alpha$ and $\beta$ are arbitrary solutions of the ODE $d f / d t \pm t / f=K$ for some fixed $K \neq 0$. (We will take the plus sign in these equations, the computation for the other sign being completely analogous.) In this case, (5.9) takes the form

$$
\begin{aligned}
& f_{Z}=\left(\frac{\alpha(f)}{u}\left(K-\frac{g}{\beta(g)}\right)-f_{u}\right) g_{Q}, \\
& g_{Z}=\left(\frac{\beta(g)}{u}\left(K-\frac{f}{\alpha(f)}\right)-g_{u}\right) f_{P} .
\end{aligned}
$$

(From now on, instead of writing $\alpha(f)$ and $\beta(g), \alpha$ and $\beta$ will be understood to be composed with $f(x, y, u, Z, P)$ and $g(x, y, u, Z, Q)$ respectively.)

Unlike in previous examples, here it is not valid to conclude that $f$ and $g$ are linear in $P$ and $Q$. In fact, differentiating (5.25) with respect to $Q$ gives

$$
0=\left(u \beta f_{u}-(K \beta-g) \alpha\right) g_{Q Q}+\frac{\left(\beta^{2}-(K \beta-g) g\right)}{\beta^{2}} \alpha g_{Q}^{2},
$$

enabling us to determine $g_{Q Q}$. (If the coefficient in front were identically zero, then $\beta(q)$ would be identically equal to a constant times $q$, which contradicts $K \neq 0$.) Similarly, differentiating (5.26) yields

$$
0=\left(u \alpha g_{u}-(K \alpha-f) \beta\right) f_{P P}+\frac{\left(\alpha^{2}-(K \alpha-f) f\right)}{\beta} f_{P}^{2} .
$$


The characteristic invariants for (IV) are

$$
J_{1}=\frac{r}{\alpha}-\frac{\alpha}{u}, \quad J_{2}=\frac{t}{\beta}-\frac{\beta}{u} .
$$

Substituting for $p$ and $r$ from (5.6) and (5.10) gives

$$
J_{1}=\frac{f_{x}+f_{u} f+f_{Z} P+f_{P} R}{\alpha}-\frac{\alpha}{u},
$$

so that $f_{P} / \alpha$ must be a function of $x$ and $P$ only. Setting the derivatives of this with respect to $u, y$, and $Z$ equal to zero yields

$$
f_{P u}=\left(K-\frac{f}{\alpha}\right) \frac{f_{P} f_{u}}{\alpha}, \quad f_{P y}=\left(K-\frac{f}{\alpha}\right) \frac{f_{P} f_{y}}{\alpha}, \quad f_{P Z}=\left(K-\frac{f}{\alpha}\right) \frac{f_{P} f_{Z}}{\alpha},
$$

where $f_{y}$ is given by (5.7) and $f_{Z}$ is given by (5.25). Similarly, from the $T$ coefficient in $J_{2}$ we get that $g_{Q} / \beta$ must be a function of $y$ and $Q$ only, and hence

$$
g_{Q u}=\left(K-\frac{g}{\beta}\right) \frac{g_{Q} g_{u}}{\beta}, \quad g_{Q x}=\left(K-\frac{g}{\beta}\right) \frac{g_{Q} g_{x}}{\beta}, \quad g_{Q Z}=\left(K-\frac{g}{\beta}\right) \frac{g_{Q} g_{Z}}{\beta} .
$$

We may also differentiate (5.25) and (5.26) to obtain equations for $f_{P x}$ and $g_{Q y}$.

We encode the various first- and second-order partial differential equations for $f$ and $g$ derived so far into an exterior differential system generated by 1-forms $\psi_{1}, \ldots, \psi_{6}$, as we did for equation (XIII). Unlike the previous example, we do not need to prolong, but instead obtain integrability conditions which take the form

$$
\alpha g_{u}=\beta f_{u},
$$

and

$$
\left(u \beta f_{u}-(K \beta-g) \alpha\right) g_{Q}=\left(u \alpha g_{u}-(K \alpha-f) \beta\right) f_{P} .
$$

(Note that, by using (5.25), (5.26), this implies that $\alpha g_{Z}=\beta f_{Z}$.) With these conditions incorporated into the EDS, it becomes involutive with last nonzero character $s_{1}=2$.

Solutions of this system may be obtained by observing that the quantities

$$
\lambda=\frac{f_{u}}{\alpha}, \quad \mu=\frac{f_{Z}}{\alpha}, \quad \gamma=\alpha-(\lambda f+\mu P) u, \quad \delta=\beta-(\lambda g+\mu Q) u
$$

must be functions of $x, y, u$ and $Z$ only, and satisfy the following compatible system of first-order PDE:

$$
\begin{array}{llrl}
\frac{\partial \lambda}{\partial u}=-u \lambda^{3}+K \lambda^{2}-\frac{2}{u} \lambda, & \frac{\partial \mu}{\partial u}=-\frac{\left(u^{2} \lambda^{2}-K u \lambda+1\right)}{u} \mu, & \frac{1}{\gamma} \frac{\partial \gamma}{\partial u}=\frac{1}{\delta} \frac{\partial \delta}{\partial u}=(K-u \lambda) \lambda, \\
\frac{\partial \lambda}{\partial Z}=\frac{\partial \mu}{\partial u}, & \frac{\partial \mu}{\partial Z}=(K-u \lambda) \mu^{2}, & \frac{1}{\gamma} \frac{\partial \gamma}{\partial Z}=\frac{1}{\delta} \frac{\partial \delta}{\partial Z}=(K-u \lambda) \mu, \\
\frac{\partial \lambda}{\partial x}=-\frac{\left(u^{2} \lambda^{2}-K u \lambda+1\right)}{u^{2}} \gamma, & \frac{\partial \mu}{\partial x}=(K-u \lambda) \frac{\gamma \mu}{u}, & \frac{\partial \delta}{\partial x}=(K-u \lambda) \frac{\gamma \delta}{u}, \\
\frac{\partial \lambda}{\partial y}=-\frac{\left(u^{2} \lambda^{2}-K u \lambda+1\right)}{u^{2}} \delta, & \frac{\partial \mu}{\partial y}=(K-u \lambda) \frac{\delta \mu}{u}, & \frac{\partial \gamma}{\partial y}=\frac{\partial \delta}{\partial x} .
\end{array}
$$


A solution $(\gamma, \delta, \lambda, \mu)$ to this PDE system may be constructed by integrating successively in the $u$-direction, the $Z$-direction, the $x$-direction and the $y$-direction. (Note that the $x$-dependence of $\gamma$ and the $y$-dependence of $\delta$ are given by arbitrary functions.) Once $\gamma$ and $\delta$ are known, they implicitly determine $f$ and $g$.

Using (5.3), one can check that the resulting Bäcklund transformations are holonomic if and only if, in the above system, $u^{2} \lambda^{2}-K u \lambda+1=0$. Thus, holonomic transformations exist, and depend on fewer arbitrary constants but the same number of arbitrary functions. For example, if $K=2$, then a solution to the above system is given by

$$
\lambda=\frac{1}{u}, \quad \mu=\frac{-1}{Z-v(x)-w(y)}, \quad \gamma=\frac{v^{\prime}(x) u}{Z-v(x)-w(y)}, \quad \delta=\frac{w^{\prime}(y) u}{Z-v(x)-w(y)} .
$$

Then, using (5.34), a holonomic Bäcklund transformation is implicitly defined by

$$
\alpha(p)-p=\frac{\left(v^{\prime}(x)-P\right) u}{Z-v(x)-w(y)}, \quad \beta(q)-q=\frac{\left(w^{\prime}(y)-Q\right) u}{Z-v(x)-w(y)} .
$$

5.4. Summary. Besides equations (IV) and (XIII) discussed above, we have also investigated the exterior differential system for Bäcklund transformations to the wave equation for equations (V), (VII), (IX), (XI) and (XII). Even if explicit formulas are not available, in each case we use the Cartan-Kähler Theorem to determine (in terms of the last nonzero Cartan character) the size of the solution set, in both the holonomic and non-holonomic cases. The results are summarized in the table below.

\begin{tabular}{|c|c|c|c|}
\hline Equation & Monge-Integrable & Holonomic BTs & Non-holonomic BTs \\
\hline I & no & yes, $s_{1}=2$ & yes, $s_{1}=2$ \\
II & no & no & yes, $s_{1}=2$ \\
III & no & no & yes, $s_{1}=2$ \\
IV & no & yes, $s_{1}=2$ & yes, $s_{1}=2$ \\
V & no & yes, $s_{1}=2$ & yes, $s_{1}=2$ \\
VI & yes & yes, $s_{2}=2$ & yes, $s_{3}=1$ \\
VII & no & no & yes, $s_{1}=2$ \\
VIII & yes & yes, $s_{2}=2$ & yes, $s_{3}=1$ \\
IX & no & no & yes, $s_{1}=2$ \\
X & yes & yes, $s_{2}=2$ & yes, $s_{3}=1$ \\
XI & no & yes, $s_{1}=2$ & no \\
XII & no & yes, $s_{1}=2$ & no \\
XIII & no & yes, $s_{1}=2$ & no \\
\hline
\end{tabular}

Note that the approach described in $\S 5.3$ is not feasible for the Monge-integrable equations (VI, VIII and X), but for completeness we include them in the table, together with the results from the analysis in $\S 4.2$. The Cartan character for the system for holonomic Bäcklund transformations for such equations is variable, depending on whether one considers the cases (1), (2), or (3), as described in $\S 4.2$.

It is interesting to note that equations (I), (IV) and (V) have both holonomic and non-holonomic transformations, in roughly the same degree of generality. In fact, it is possible that these two kinds of Bäcklund transformations linking the same pair of equations may be closely related. In our previous paper [4], we pointed out that the transformation (Z.I) is a composition of two simpler transformations, a holonomic Bäcklund transformation to the wave equation, and a contact transformation from 
the wave equation to itself. It is possible that, more generally, the non-holonomic transformations for these equations are obtainable from holonomic transformations in this way.

6. Concluding Remarks. In this section, we will indicate some interesting directions in which the results in this paper might be extended, and some important questions about Bäcklund transformations to which the techniques in this paper may be relevant.

1. The set of equivalence classes (under contact transformations) of secondorder Monge-Ampère equations to which the results of $\S 3$ in this paper apply is relatively small, confined to the equations on the Goursat-Vessiot list. It would be interesting to see if the arguments in that section could be applied to hyperbolic systems of class $k>1$. In other words, given a hyperbolic system $\mathcal{I}$ of class $k$, linked to the standard wave equation by a Bäcklund transformation, can one prove that the prolongation of $\mathcal{I}$ is Darboux-integrable? Likewise, given a hyperbolic EDS $\mathcal{I}$ of class $k$, such that its prolongation is Darbouxintegrable, does there exist a Bäcklund transformation between $\mathcal{I}$ and the Monge-Ampère system for the standard wave equation? (The argument given at the end of Chapter 7 in [8] shows that there is a Bäcklund transformation between the wave equation and the prolongation of $\mathcal{I}$; however, for practical purposes it is desirable to have a Bäcklund transformation between systems of as low an order as possible, so that one has a smaller system of ODE to solve in order to construct solutions.) These hyperbolic systems would include, for example, the Monge-Ampère equations which are Darboux-integrable at third order, which have not been classified and are thought to comprise a much larger set.

2. It is a theorem of Sophus Lie that no Monge-Ampère equation of the form $u_{x y}=f(u)$ is Darboux-integrable (after arbitrary many prolongations) except when $f(u)=\exp (a u+b)$ for constants $a$ and $b$ (see [6], Chapter IX). Consequently, important equations like sine-Gordon cannot have a Bäcklund transformation to the wave equation. Instead, the Bäcklund transformation (1.1) for sine-Gordon produces solutions to the same PDE as we started with. This is known as an auto-Bäcklund transformation; ${ }^{2}$ such transformations play an important role in the theory of completely integrable PDE [9].

It is therefore of interest to try to identify those Monge-Ampère equations which have non-trivial auto-Bäcklund transformations. We remark that for such transformations, the Monge-Ampère systems $\mathcal{I}$ on $\mathcal{M}$ and $\overline{\mathcal{I}}$ on $\overline{\mathcal{M}}$ must be contact-equivalent, i.e., there must be a diffeomorphism $\Phi: \mathcal{M} \rightarrow \overline{\mathcal{M}}$ which pulls back $\overline{\mathcal{I}}$ to $\mathcal{I}$.

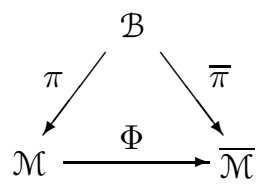

Necessary conditions for the existence of such a diffeomorphism may be derived from the fact that it is required to preserve the differential invariants of

\footnotetext{
${ }^{2}$ This terminology is not universally accepted; Hongyou Wu [12] has proposed that transformations between different PDEs be known as Miura transformations, and the term Bäcklund transformation be reserved for what we are calling auto-Bäcklund transformations.
} 
the Monge-Ampère systems. (See [2], §2.1, for a derivation of these invariants using the method of equivalence.)

3. Our previous paper [4] began the exploration of parametric Bäcklund transformations using the method of equivalence. Such transformations contain an arbitrary parameter in the Bäcklund system; for example, an arbitrary nonzero parameter $\lambda$ may be interpolated in the sine-Gordon auto-Bäcklund transformation (1.1) to give

$$
\begin{aligned}
& v_{x}-u_{x}=\frac{\lambda}{2} \sin ((u+v) / 2), \\
& v_{y}+u_{y}=-\frac{1}{2 \lambda} \sin ((u-v) / 2) .
\end{aligned}
$$

One observes that this system differs from (1.1) merely by scaling $x$ by $\lambda$ and $y$ by $\lambda^{-1}$-a change of variables which is a symmetry of the sine-Gordon equation but not of the system (1.1). This scaling symmetry can also be applied to the Bäcklund transformation (1.2), to produce a parametric transformation

$$
z_{x}=u_{x}-2 \lambda \exp ((u+z) / 2), \quad z_{y}=-u_{y}+\frac{1}{\lambda} \exp ((u-z) / 2),
$$

where $u(x, y)$ satisfies Liouville's equation and $z(x, y)$ solves the wave equation. (In fact, this transformation is derived from the most general form (5.18) by setting $k=1$ and choosing $v(x)=2 \ln \lambda$ and $w(y)=0$.) In [4] it is shown that these transformations can be generated from a non-parametric Bäcklund transformation by starting with a symmetry vector field on $\mathcal{M}$, choosing a lift into $\mathcal{B}^{6}$ which is not a symmetry of the Pfaffian system $\mathcal{J}$, but such that pulling $\mathcal{J}$ back to $\mathcal{B} \times \mathbb{R}$ via the 1-parameter family of diffeomorphisms generated by the lift gives a family of transformations. The same approach can be taken with other transformations discussed in $\S 4.1$; for example, the transformation (Z.II) may be generalized to a parametric transformation

$$
z z_{x}-\lambda u u_{x}=\sqrt{z^{2}-\lambda u^{2}} \sqrt{\lambda+z_{x}^{2}}, \quad z z_{y}-\lambda u u_{y}=\sqrt{z^{2}-\lambda u^{2}} \sqrt{\lambda+z_{y}^{2}}
$$

for $\lambda>0$, where $u$ satisfies (II) and $z$ solves the wave equation. (This is obtained by starting with the symmetry of (II) that simultaneously scales $u$, $x$ and $y$.)

With these examples in evidence, and given the importance of parametric Bäcklund transformations in the study of 'soliton' equations, it is desirable to characterize those transformations that may be made to depend on an arbitrary parameter by lifting symmetry vector fields.

\section{REFERENCES}

[1] M. Biesecker, Geometric Studies in Hyperbolic Systems in the Plane, Ph.D. thesis, Utah State University, 2003.

[2] R. Bryant, P. Griffiths and D. Grossmann, Exterior differential systems and EulerLagrange partial differential equations, University of Chicago Press, 2003.

[3] J. Clelland, Homogeneous Bäcklund transformations of hyperbolic Monge-Ampère systems, Asian J. Math., 6 (2002), pp. 433-480.

[4] J. Clelland and T. Ivey, Parametric Bäcklund transformations I: Phenomenology, Trans. Amer. Math. Soc., 357 (2005), pp. 1061-1093. 
[5] R. Gardner, The Method of Equivalence and Its Applications, CBMS-NSF Regional Conf. Ser. in Appl. Math. 58, SIAM, Philadelphia, 1989.

[6] E. Goursat, Leçons sur l'intégration des équations aux dérivées partielles du second ordre, Gauthier-Villars, 1896-1898.

[7] -, Recherches sur quelques équations aux dérivées partielles du second ordre, Annales de la Faculté de Toulouse (deuxième serie), 1 (1899), pp. 31-78.

[8] T. Ivey and J. M. Landsberg, Cartan for Beginners: Differential geometry via moving frames and exterior differential systems (with J.M. Landsberg), Graduate Studies in Mathematics vol. 61, American Mathematical Society, 2003.

[9] A. P. Fordy, A Historical Introduction to Solitons and Bäcklund Transformations, in "Harmonic Maps and Integrable Systems" (ed. Fordy and Wood), Vieweg, 1994.

[10] M. JuRÁš, Geometric Aspects of Second-Order Partial Differential Equations in the Plane, Ph.D. thesis, Utah State University, 1997.

[11] E. Vessiot, Sur les équations aux dérivées partielle du second ordre intégables par la methode de Darboux, J. Math Pures Appl., 18 (1939), pp. 1-61 and 21 (1942), pp. 1-66.

[12] X. CAO, H. WU AND C. XU, On Miura transformations among nonlinear partial differential equations, J. Math. Phys., 47:8 (2006).

[13] M. Yu. Zvyagin, Classification of Bäcklund transformation of Second-Order Partial Differential Equations, Mat. Zametki, 29 (1981), pp. 829-842 (English translation: Math. Notes, 29 (1981), pp. 422-429).

[14] -, Second order equations reducible to $z_{x y}=0$ by a Bäcklund transformation, Dokl. Akad. Nauk SSSR, 316 (1991), pp. 36-40 (English translation: Soviet Math. Dokl., 43 (1991), pp. 30-34). 